\title{
Density Functional Theory of doped superfluid liquid helium and nanodroplets
}

\author{
Francesco Ancilotto, ${ }^{1,2}$ Manuel Barranco, ${ }^{3,4,5}$ François Coppens, ${ }^{3}$ Jussi Eloranta, ${ }^{6}$ \\ Nadine Halberstadt, ${ }^{3}$ Alberto Hernando, ${ }^{7}$ David Mateo, ${ }^{8}$ and Martí $\mathrm{Pi}^{4,5}$ \\ ${ }^{1}$ Dipartimento di Fisica e Astronomia "Galileo Galilei" and CNISM, \\ Università di Padova, via Marzolo 8, 35122 Padova, Italy \\ ${ }^{2}$ CNR-IOM Democritos, via Bonomea, 265 - 34136 Trieste, Italy \\ ${ }^{3}$ Université Toulouse 3 and CNRS, Laboratoire des Collisions, \\ Agrégats et Réactivité, IRSAMC, 118 route de Narbonne, \\ F-31062 Toulouse Cedex 09, France \\ ${ }^{4}$ Departament FQA, Facultat de Física, \\ Universitat de Barcelona. Diagonal 645, 08028 Barcelona, Spain \\ ${ }^{5}$ Institute of Nanoscience and Nanotechnology (IN2UB), Universitat de Barcelona. \\ ${ }^{6}$ Department of Chemistry and Biochemistry, \\ California State University at Northridge, California 91330, USA \\ ${ }^{7}$ Social Thermodynamics Applied Research (SThAR) EPFL \\ Innovation Park Bâtiment C, CH-1015 Lausanne, Switzerland \\ ${ }^{8}$ Applied Complexity Group, Singapore University of \\ Technology and Design. 8 Somapah Road, Singapore 487372
}

(Dated: October 16, 2018) 


\begin{abstract}
During the last decade, density function theory (DFT) in its static and dynamic time dependent forms, has emerged as a powerful tool to describe the structure and dynamics of doped liquid helium and droplets. In this review, we summarize the activity carried out in this field within the DFT framework since the publication of the previous review article on this subject [M. Barranco et al., J. Low Temp. Phys. 142, 1 (2006)]. Furthermore, a comprehensive presentation of the actual implementations of helium DFT is given, which have not been discussed in the individual articles or are scattered in the existing literature. This is an Accepted Manuscript of an article published on August 2, 2017 by Taylor \& Francis Group in Int. Rev. Phys. Chem. 36, 621 (2017), available online: http://dx.doi.org/10.1080/0144235X.2017.1351672
\end{abstract}




\section{Contents}

PAGE

I. Introduction 4

II. Density functional theory of liquid ${ }^{4} \mathrm{He}$ at zero temperature 6

II.A. Theoretical basis of density functional theory 6

II.B. The Orsay-Trento density functional 8

II.C. Recent improvements of the OT-DFT functional 12

II.C.1. The 'solid' density functional 12

II.C.2. Instability of the backflow term 14

III. Time-independent calculations

III.A. General considerations 17

III.B. Introduction of vorticity

IV. Dynamics 21

IV.A. Heavy impurities $\quad 22$

IV.B. Test particle method for light impurities 26

IV.C. Simulation of absorption and emission spectra using the density fluctuation method 29

V. Recent applications of DFT for impurity doped superfluid helium

V.A. Alkali metal doped helium droplets: solvation and absorption spectra 34

V.B. Alkaline earth metal doped helium droplets: solvation and absorption spectra 39

V.C. Droplets doped with more than one species 42

V.D. Cluster-doped helium droplets 44

V.E. Doped mixed ${ }^{3} \mathrm{He}-{ }^{4} \mathrm{He}$ and ${ }^{3} \mathrm{He}$ droplets 46

V.F. Electrons in liquid helium 52

V.G. Cations in liquid helium and droplets 57

V.H. Intrinsic helium impurities 63

V.I. Translational motion of ions below the Landau critical velocity 66

V.J. Critical Landau velocity in small ${ }^{4} \mathrm{He}$ droplets 68

V.K. Rotational superfluidity 70

V.L. Interaction of impurities with vortex lines $\quad 71$

$\begin{array}{ll}\text { V.L.1. Electrons } & 72\end{array}$ 
V.L.2. Atomic and molecular impurities

V.M. Vortex arrays in ${ }^{4} \mathrm{He}$ droplets $\quad 77$

V.N. Dynamics of alkali atoms excited on the surface of ${ }^{4}$ He droplets 81

V.O. Capture of impurities by ${ }^{4} \mathrm{He}$ droplets 88

V.P.1. Pure droplets

V.P.2. Droplets hosting vortices 93

V.Q. Liquid helium on nanostructured surfaces 94

V.R. Soft-landing of helium droplets 96

VI Summary and outlook

\section{INTRODUCTION}

Liquid helium-4 becomes superfluid below the lambda transition at $2.17 \mathrm{~K}$ due to partial Bose-Einstein condensation (BEC). It exhibits unusual macroscopic behavior such as e.g. vanishing viscosity and the thermo-mechanical effect 1 On the atomic scale, the response of this fascinating quantum liquid has been studied experimentally by using solvated atomic and molecular species as probes. ${ }^{2}$ The early experiments employed bulk liquid helium samples where only ionic species and intrinsic helium excimers could be introduced. A breakthrough in this area has been the development of the helium droplet technique, which made it possible to embed neutral atomic and molecular species in superfluid helium droplets at $0.37 \mathrm{~K} \cdot \sqrt[344]{1 n}$ addition to their intrinsic interest as a superfluid object of finite size, helium droplets provide an ideal matrix for spectroscopic experiments due to their low temperature and weak interaction with the solvated species. ${ }^{[521}$

From the theoretical point of view, superfluid helium must be considered as a high dimensional quantum system. Quantum Monte Carlo (QMC) ${ }^{21}$ and direct quantum mechanical $22 \sqrt{24}$ calculations are the most accurate methods, but their computational demand quickly exceeds currently available computer resources when the number of helium atoms increases. Furthermore, QMC cannot describe dynamic evolution of superfluid helium in real time. To address these limitations, approximate methods based on density functional theory (DFT) formalism have been introduced ${ }^{25}$ DFT can be applied to much 
larger systems than QMC and allows for time-dependent formulation. As such, it offers a good compromise between accuracy and computational feasibility. The main drawback of DFT is that the exact energy functional is not known and must therefore be constructed in a semi-empirical manner. Nevertheless, DFT is the only method to date that can successfully reproduce results from a wide range of time-resolved experiments in superfluid helium on the atomic scale.

Application of recently developed femtosecond laser techniques to study helium droplets $\frac{15 \mid 28}{10}$ highlights the importance of time-dependent DFT (TDDFT). For example, TDDFT can be used to analyze experiments that employ free electron laser pulses to visualize vortex arrays in helium droplets, $\stackrel{29}{29}$ or the dynamics following optical excitation of guest atoms or molecules embedded in helium droplets. $\stackrel{30131}{ }$ It is the only method that allows for such a close interplay between theory and time-resolved helium droplet experiments. In fact, many of the results presented in this review were obtained as joint experimental-theoretical collaborative work.

Despite the wide success of both DFT and TDDFT, they have known limitations, especially when the interaction between the guest species and helium is strong. ${ }^{32[33} \mathrm{New}$ strategies for resolving with such problems are also summarised in this review. In addition, applications of DFT and time-dependent DFT will be reviewed with a focus on the new developments that have appeared after the previous review article on this topic. ${ }^{7}$

We provide a comprehensive presentation of the most recent DFT models and their applications to superfluid helium droplets and bulk liquid. Selected topics dealing with DFT of non-superfluid ${ }^{3} \mathrm{He}$ are also briefly discussed; some practical details of the DFT implementation are given in Ref. 34. As stated by Frank Stienkemeier and Kevin Lehmann in their 2006 topical review, ${ }^{9}$ a truly comprehensive review of the activity carried out recently in this field would require a monograph instead of a review article; the reader is thus referred to the appropriate literature, in particular to some recent reviews ${ }^{6} \sqrt{15128}$ for the subjects not considered in detail here. 


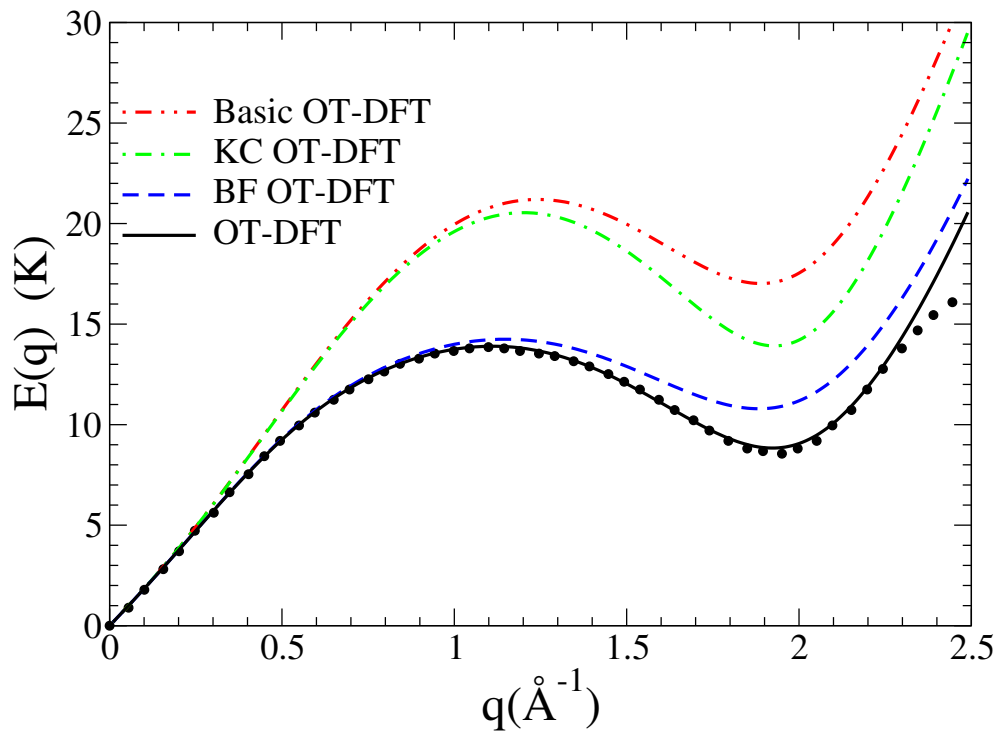

FIG. 1. Dispersion relation for elementary excitations in liquid ${ }^{4} \mathrm{He}$ calculated as in Ref. 35. 'Basic' indicates the OT-DFT without the non-local kinetic energy correlation (KC) nor the backflow (BF) terms; KC OT-DFT adds to the basic OT-DFT the KC term; BF OT-DFT adds to the basic OTDFT the BF term. The dots are the experimental data from Ref. 36. The Landau velocity $v_{L}=E(q) /\left.(\hbar q)\right|_{\min }$ obtained for each functional is $60.3 \mathrm{~m} / \mathrm{s}(\mathrm{OT}-\mathrm{DFT}) ; 75.1 \mathrm{~m} / \mathrm{s}$ (BF OT-DFT); $94.4 \mathrm{~m} / \mathrm{s}$ (KC OT-DFT); $118 \mathrm{~m} / \mathrm{s}$ (basic OT-DFT); and 57.5 (experiment).

\section{DENSITY FUNCTIONAL THEORY OF LIQUID ${ }^{4}$ HE AT ZERO TEMPERA- TURE}

\section{A. Theoretical basis of density functional theory}

The starting point is the Hohenberg-Kohn (HK) theorem, ${ }^{37}$ which states that the total energy $E$ of a many-body quantum system at $T=0$ is a functional of the one-particle density $\rho(\mathbf{r})=\left\langle\Phi\left|\sum_{i} \delta\left(\mathbf{r}-\mathbf{r}_{i}\right)\right| \Phi\right\rangle$ ( $\Phi$ being the many-body wave function):

$$
E[\rho]=\mathcal{T}[\rho]+\int d \mathbf{r} \mathcal{E}[\rho]
$$

where the kinetic energy functional has been separated from the interaction part.

The Kohn-Sham formulation ${ }^{38}$ of the HK theorem allows to write the above functional 
in the form

$$
E[\rho]=T[\rho]+\int d \mathbf{r} \mathcal{E}_{c}[\rho]
$$

where $T[\rho]$ is the kinetic energy of a fictitious system of non-interacting particles, with the same density of the original one, described by single-particle orbitals $\left\{\phi_{i}(\mathbf{r})\right\}$

$$
T=-\frac{\hbar^{2}}{2 m_{4}} \sum_{i} \int d \mathbf{r} \phi_{i}^{*}(\mathbf{r}) \nabla^{2} \phi_{i}(\mathbf{r})
$$

The sum extends to the $N_{4}$ particles of mass $m_{4}$ in the system. The difference $\mathcal{T}[\rho]-T[\rho]$ has been buried in the interaction term $\mathcal{E}_{c}$. The density of such non-interacting system is thus $\rho(\mathbf{r})=\sum_{i}\left|\phi_{i}(\mathbf{r})\right|^{2}$. We conform here to the common notation used for DFT studies of helium systems, ${ }^{27}$ which defines as 'correlation energy density' the functional $\mathcal{E}_{c}$, even if it includes also He-He interactions at the mean-field level (first term in Eq. (8) below).

Assuming complete Bose-Einstein condensation at $T=0$ (i.e. all the ${ }^{4} \mathrm{He}$ atoms are in the same single-particle orbital $\left.\phi_{0}\right)$, the many-body wave function is simply

$$
\Phi\left(\mathbf{r}_{1}, \mathbf{r}_{2}, \cdots \mathbf{r}_{N_{4}}\right)=\prod_{i=1}^{i=N_{4}} \phi_{0}\left(\mathbf{r}_{i}\right)
$$

while $\rho(\mathbf{r})=N_{4}\left|\phi_{0}(\mathbf{r})\right|^{2}$. Although the actual condensate fraction of superfluid ${ }^{4} \mathrm{He}$ is less than $10 \%$, the available helium density functionals have been devised such that, by starting from a fully condensed state, the interaction term $\mathcal{E}_{c}$ allows to reproduce the relevant physical properties of liquid helium at $T=0$.

It is customary to define an order parameter $\Psi$ (also called effective wave function) as $\Psi(\mathbf{r})=\sqrt{N_{4}} \phi_{0}(\mathbf{r})$. The kinetic energy of the condensate is thus

$$
T[\rho]=-\frac{\hbar^{2}}{2 m_{4}} N_{4}\left\langle\phi_{0}\left|\nabla^{2}\right| \phi_{0}\right\rangle=\frac{\hbar^{2}}{2 m_{4}} \int d \mathbf{r}|\nabla \Psi|^{2}
$$

The Runge-Gross theorem extends DFT to describe the time evolution of the system through the time-dependent DFT (TDDFT) formalism. ${ }^{39}$ In this case, functional variation of the associated Lagrangian leads to a time-dependent Euler-Lagrange (EL) equation

$$
\imath \hbar \frac{\partial}{\partial t} \Psi(\mathbf{r}, t)=\left\{-\frac{\hbar^{2}}{2 m_{4}} \nabla^{2}+\frac{\delta \mathcal{E}_{c}}{\delta \rho}\right\} \Psi(\mathbf{r}, t) \equiv \mathcal{H}[\rho] \Psi(\mathbf{r}, t)
$$

Given the initial state $\Psi(\mathbf{r}, 0)$, solution of this non-linear equation yields $\Psi(\mathbf{r}, t)$ which, in the hydrodynamic picture $\stackrel{40}{,}$ can be decomposed into liquid density and the associated velocity 
potential field. For stationary states, $\Psi(\mathbf{r}, t)=\Psi_{0}(\mathbf{r}) e^{-1 \mu t / \hbar}$ and Eq. (6) can be cast into a non-linear time-independent EL equation

$$
\left\{-\frac{\hbar^{2}}{2 m_{4}} \nabla^{2}+\frac{\delta \mathcal{E}_{c}}{\delta \rho}\right\} \Psi_{0}(\mathbf{r})=\mu \Psi_{0}(\mathbf{r})
$$

where $\mu$ is the chemical potential. Iterative solution of Eq. (7) determines the particle density $\rho(\mathbf{r})=\left|\Psi_{0}(\mathbf{r})\right|^{2}$ (a similar relationship holds in the time-dependent situation) and hence the total energy of the system.

In its time-independent formulation, DFT is a ground-state theory. However, within the HK theorem, the variational principle is applicable to the lowest state of a given symmetry, which may be different from the true ground state of the system. For example, this can be employed to obtain stationary vortex solutions in helium droplets by DFT. Similarly, minimization of the energy functional in the presence of additional constraints (e.g., fixed total angular momentum) will provide the correct density for the associated excited state. In particular, this technique can be used to produce vortex arrays in helium droplets. However, for general excited states, there is no equivalent HK theorem and TDDFT must be used to model them.

In the case of phenomenological helium DFT, the quality of the results depends on the functional form used. As an example, TDDFT calculation of the dispersion relation for uniform liquid helium (an excited state property) is shown in Fig. 1. The OT-DFT introduced below gives results in agreement, by construction, with the experimental ('exact') results. This obviously does not guarantee that the same functional would also give reliable results for inhomogeneous systems. However, based on our experience, these functionals are highly 'transferable' to such situations and provide results that are generally in good agreement with experiments.

Approximate representations for the interaction energy density functional $\mathcal{E}_{c}$, which are capable of describing inhomogeneous ${ }^{4} \mathrm{He}$ systems quantitatively, are discussed in the following Section.

\section{B. The Orsay-Trento density functional}

The first and simplest DFT model for superfluid ${ }^{4} \mathrm{He}$ was developed by Stringari and coworkers. ${ }^{25 \mid 26}$ In this approach, $\mathcal{E}_{c}[\rho]$ consists of a sum of terms that only depend on the 


\begin{tabular}{cccccc}
\hline \hline$\epsilon_{L J}(\mathrm{~K})$ & $\sigma(\AA)$ & $h(\AA)$ & $c_{2}\left(\mathrm{~K} \AA^{6}\right)$ & $c_{3}\left(\mathrm{~K} \AA^{9}\right)$ & $\alpha_{s}\left(\AA^{3}\right)$ \\
10.22 & 2.556 & 2.190323 & $-2.41186 \times 10^{4}$ & $1.85850 \times 10^{6}$ & 54.31 \\
\hline$\rho_{0 s}\left(\AA^{-3}\right)$ & $l(\AA)$ & $C$ (Hartree) & $\beta\left(\AA^{3}\right)$ & $\rho_{m}\left(\AA^{-3}\right)$ & $\gamma_{11}$ \\
0.04 & 1. & 0.1 & 40. & 0.37 & -19.7544 \\
\hline$\gamma_{12}\left(\AA^{-2}\right)$ & $\alpha_{1}\left(\AA^{-2}\right)$ & $\gamma_{21}$ & $\gamma_{22}\left(\AA^{-2}\right)$ & $\alpha_{2}\left(\AA^{-2}\right)$ & \\
12.5616 & 1.023 & -0.2395 & 0.0312 & 0.14912 & \\
\hline \hline
\end{tabular}

TABLE I. Model parameters for the OT-DFT and solid functionals.

local density $\rho(\mathbf{r})$. More recent models include also finite-range and non-local terms, which greatly improve the accuracy of the method, especially when applied to highly inhomogeneous systems.

The most successful approach to date is the finite range, non-local Orsay-Trento DFT model (OT-DFT) $\stackrel{27}{27}$ which has been calibrated to reproduce bulk liquid properties such as the energy per atom, the equilibrium density, the dispersion relation, and the compressibility at $P=T=0$. The OT-DFT energy functional is written as

$$
\begin{aligned}
\mathcal{E}_{c}[\rho, \mathbf{v}]= & \frac{1}{2} \int \mathrm{d} \mathbf{r}^{\prime} \rho(\mathbf{r}) V_{L J}\left(\left|\mathbf{r}-\mathbf{r}^{\prime}\right|\right) \rho\left(\mathbf{r}^{\prime}\right) \\
& +\frac{1}{2} c_{2} \rho(\mathbf{r})[\bar{\rho}(\mathbf{r})]^{2}+\frac{1}{3} c_{3} \rho(\mathbf{r})[\bar{\rho}(\mathbf{r})]^{3} \\
& -\frac{\hbar^{2}}{4 m_{4}} \alpha_{s} \int \mathrm{d} \mathbf{r}^{\prime} F\left(\left|\mathbf{r}-\mathbf{r}^{\prime}\right|\right)\left[1-\frac{\tilde{\rho}(\mathbf{r})}{\rho_{0 s}}\right] \nabla \rho(\mathbf{r}) \cdot \nabla^{\prime} \rho\left(\mathbf{r}^{\prime}\right)\left[1-\frac{\tilde{\rho}\left(\mathbf{r}^{\prime}\right)}{\rho_{0 s}}\right] \\
& -\frac{m_{4}}{4} \int d \mathbf{r}^{\prime} V_{J}\left(\left|\mathbf{r}-\mathbf{r}^{\prime}\right|\right) \rho(\mathbf{r}) \rho\left(\mathbf{r}^{\prime}\right)\left[\mathbf{v}(\mathbf{r})-\mathbf{v}\left(\mathbf{r}^{\prime}\right)\right]^{2}
\end{aligned}
$$

The first term corresponds to a classical Lennard-Jones interaction between helium atoms, which is truncated at short distances where the correlation effects become important

$$
\begin{aligned}
V_{L J}(r) & =4 \epsilon_{L J}\left[\left(\frac{\sigma}{r}\right)^{12}-\left(\frac{\sigma}{r}\right)^{6}\right] \quad \text { if } r>h \\
& =0 \quad \text { otherwise }
\end{aligned}
$$

The second line in Eq. (8) accounts for short-range correlation effects. The third line (' $\alpha_{s}$ term') is a non-local kinetic energy correction $(\mathrm{KC})$ - which partially accounts for the difference $\mathcal{T}[\rho]-T[\rho]$ in the interaction term $\mathcal{E}_{c}$ - and the last term is the backflow (BF) contribution that affects the dynamic response of the functional. Note that the BF term only contributes when the order parameter is a complex valued function (e.g. time-dependent 
problem or vortex state). The velocity $\mathbf{v}(\mathbf{r})$ is determined from the current

$$
\mathbf{j}(\mathbf{r})=-\frac{\imath \hbar}{2 m_{4}}\left[\Psi^{*}(\mathbf{r}) \nabla \Psi(\mathbf{r})-\Psi(\mathbf{r}) \nabla \Psi^{*}(\mathbf{r})\right]
$$

as $\mathbf{v}(\mathbf{r})=\mathbf{j}(\mathbf{r}) / \rho(\mathbf{r})=\hbar / m_{4} \times \operatorname{Im}\{\nabla \Psi(\mathbf{r}) / \Psi(\mathbf{r})\}$. The two coarse-grained averages of the liquid density, $\bar{\rho}$ and $\tilde{\rho}$, entering into the short-range correlation terms in Eq. (8), are given by

$$
\bar{\rho}(\mathbf{r})=\int \mathrm{d} \mathbf{r}^{\prime} \rho\left(\mathbf{r}^{\prime}\right) w\left(\left|\mathbf{r}-\mathbf{r}^{\prime}\right|\right)
$$

where

$$
\begin{aligned}
w(r) & =\frac{3}{4 \pi h^{3}} \quad \text { if } \quad r<h \\
& =0 \quad \text { otherwise. }
\end{aligned}
$$

and

$$
\tilde{\rho}(\mathbf{r})=\int \mathrm{d} \mathbf{r}^{\prime} \rho\left(\mathbf{r}^{\prime}\right) F\left(\left|\mathbf{r}-\mathbf{r}^{\prime}\right|\right)
$$

where $F(r)$ is a Gaussian kernel

$$
F(r)=\frac{1}{\pi^{3 / 2} l^{3}} \mathrm{e}^{-r^{2} / l^{2}}
$$

The function $V_{J}(r)$ presents in the backflow term is defined as

$$
V_{J}(r)=\left(\gamma_{11}+\gamma_{12} r^{2}\right) e^{-\alpha_{1} r^{2}}+\left(\gamma_{21}+\gamma_{22} r^{2}\right) e^{-\alpha_{2} r^{2}}
$$

The various parameters entering the OT-DFT functional are specified in Table I.

While OT-DFT can model the response of superfluid helium very accurately, it is seldom applied to inhomogeneous systems due to its complexity. ${ }^{41}$ Furthermore, in most timedependent applications, both the kinetic energy correlation and backflow terms are often neglected because their evaluation is time consuming and they tend to exhibit numerical instabilities, especially for highly inhomogeneous systems. Strategies for overcoming these instabilities are presented in the next section.

The backflow and non-local kinetic energy correlation terms in OT-DFT are required for a quantitative description of the elementary excitation spectrum of superfluid helium. While both terms influence the energetics of the roton minimum, the backflow term has the most important contribution of the two as demonstrated in Fig. 1. Note that the Landau critical velocity predicted by the functional, which determines the onset of bulk dissipative 
behavior in time-dependent applications, is the slope of a straight line passing through the origin and tangent to the dispersion curve near the roton minimum. ${ }^{2742]}$ The influence of these terms to the description of a vortex line structure is discussed in Ref. 43, see also Sec. IIC2 below.

The accuracy of OT-DFT can be further assessed by comparing the obtained density profiles of pure helium droplets against QMC calculations. By way of an example, such a comparison is shown in Fig. 2 for a droplet with $N_{4}=50$. Since DFT should generally work better when the number of particles increases, OT-DFT will retain its accuracy for the typical droplet sizes produced in experiments (a few thousand ${ }^{4} \mathrm{He}$ atoms). Even with the kinetic energy correlation term omitted (i.e. $\alpha_{s}=0$ ), the agreement with QMC remains rather good as demonstrated in Fig. 2.

In contrast to DFT employing local functionals, the performance of finite-range functionals is superior when processes such as atomic/molecular impurity solvation or their spectroscopy is considered (see e.g. Fig. 1 of Ref. 44). Any process that requires the correct liquid response on the Ångström-scale must employ a finite range, non-local model. However, in some applications the non-local terms are not very important and it is possible to use the much simpler local functionals. Local density functionals of different complexity have been used to describe static and dynamic properties of pure and doped superfluid helium. ${ }^{45+49}$ Very recently, a zero-range reduction of the OT functional has also been applied to study inelastic scattering of Xe atoms by quantised vortices in superfluid helium. 50

The original OT-DFT formulation only applies to superfluid ${ }^{4} \mathrm{He}$ at $T=0$. It has been extended up to $T=3 \mathrm{~K}$ by considering the wetting properties of various metals, $\frac{51}{51}$ see also Ref. 52 and 53. A non-local extension of the functional has also been introduced for mixed ${ }^{3} \mathrm{He}^{-}{ }^{4} \mathrm{He}$ systems.${ }^{54}$ The latter model has been used recently to study elementary excitations of superfluid ${ }^{3} \mathrm{He}-{ }^{4} \mathrm{He}$ mixtures ${ }^{35}$ and to study the solvation of OCS in mixed ${ }^{3} \mathrm{He}-{ }^{4} \mathrm{He}$ droplets. ${ }^{[55}$ Various functionals have also been developed for pure ${ }^{3} \mathrm{He}$, see e.g. Refs. 56 and $[57$ and references therein. Finally, we note that a method similar to the one used for superfluid ${ }^{4} \mathrm{He}$ has also been used to describe cold dipolar Bose gase ${ }^{58}$ and para-hydrogen clusters, for which a DFT-based approach is also available ${ }^{[59}$ 


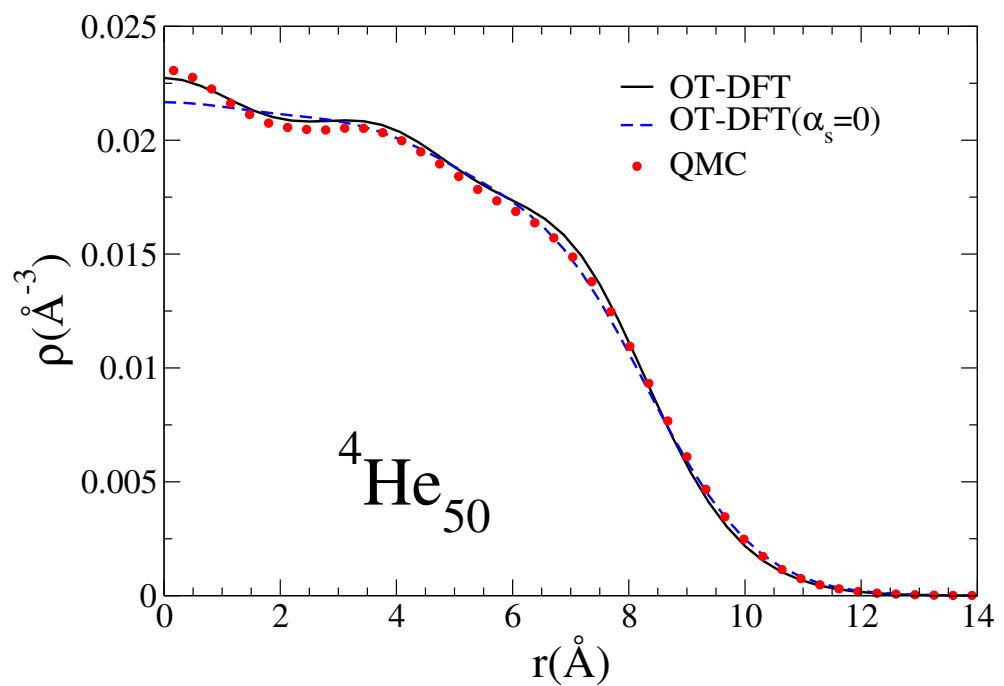

FIG. 2. Comparison between OT-DFT and QMC calculations for the density profile of the ${ }^{4} \mathrm{He}_{50}$ droplet. The QMC calculations have been carried out by M. Rossi, University of Padova.

\section{Recent improvements of the OT-DFT functional}

\section{The 'solid' density functional}

The OT-DFT functional becomes unstable in the presence of highly inhomogeneous liquid density distributions, like those occurring e.g. for the solvation of cations inside ${ }^{4}$ He. To overcome this problem, an additional cutoff term, which was originally developed to account for the liquid-solid phase transition of ${ }^{4} \mathrm{He}, \frac{60 \mid 61}{\sqrt{6}}$ can be employed to it. This is essentially a penalty term that prevents excessive liquid density accumulation

$$
\mathcal{E}_{\text {sol }}[\rho(\mathbf{r})]=C \rho(\mathbf{r})\left\{1+\tanh \left(\beta\left[\rho(\mathbf{r})-\rho_{\mathrm{m}}\right]\right)\right\}
$$

Since $\mathcal{E}_{\text {sol }}[\rho]$ is only significant when the liquid density is comparable to $\rho_{\mathrm{m}}$ or larger, it does not alter the original OT-DFT functional at densities lower than the (large) cutoff value $\rho_{\mathrm{m}}$. For instance, the total energy of pure ${ }^{4} \mathrm{He}_{1000}$ droplet is $-5400.34 \mathrm{~K}$ where the contribution of the penalty term is only $4.2 \times 10^{-5} \mathrm{~K}$. The model parameters used are specified in Table I.

Inclusion of the 'solid' term in the OT-DFT model has made it possible to use it in 


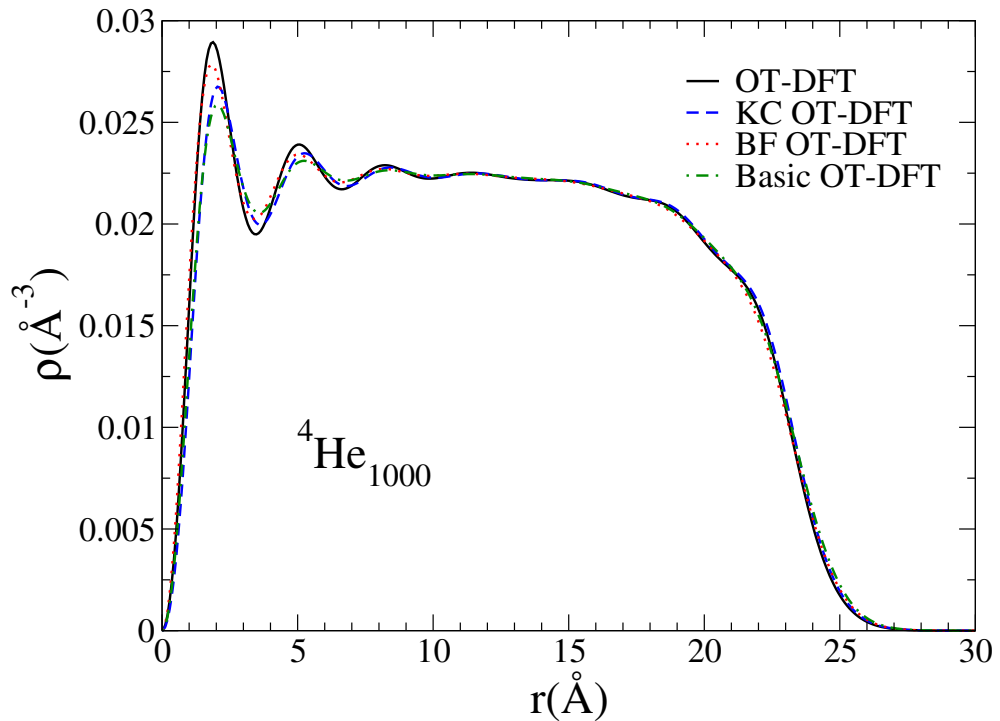

FIG. 3. Density profile of the ${ }^{4} \mathrm{He}_{1000}$ droplet hosting a linear vortex along its diameter. As in Fig. 1, the calculations have been carried out using the full OT-DFT or including only some of its terms.

complex situations where the impurity-helium interaction is strongly attractive. However, while $\mathcal{E}_{\text {sol }}[\rho]$ in Eq. (16) can prevent the unphysical density pile-up, it cannot eliminate the often observed spontaneous symmetry breaking of the ${ }^{4} \mathrm{He}$ order parameter in the presence of strongly attractive external potentials. For instance, the numerical solution can become non-spherical even when the external potential is strictly spherically symmetric. Taking a spherical average of the symmetry broken solution appears however to yield results very close to QMC calculations. ${ }^{33162}$ It is not clear at the moment how to preserve the desired symmetry during the calculations. Note that a spontaneous symmetry breaking is expected to occur around very attractive impurities, which form 'snowball' structures with a solidlike first solvation layer. The solid OT-DFT functional, consisting of $\mathcal{E}_{\text {sol }}$ and the first three terms of Eq. (8), has often been used in the static and dynamic applications discussed in the next sections. 


\begin{tabular}{|c|c|c|c|c|c|}
\hline $\begin{array}{c}P \\
\text { (bar) }\end{array}$ & $\begin{array}{c}\rho \\
\left(10^{-2} \AA^{-3}\right)\end{array}$ & $\begin{array}{c}q_{R}(\exp ) \\
\left(\AA^{-1}\right)\end{array}$ & $\begin{array}{c}\Delta(\exp ) \\
(\mathrm{K})\end{array}$ & $\begin{array}{c}q_{R}(\mathrm{OT}) \\
\left(\AA^{-1}\right)\end{array}$ & $\begin{array}{c}\Delta(\mathrm{OT}) \\
(\mathrm{K})\end{array}$ \\
\hline 0 & 2.1836 & 1.93 & 8.62 & 1.92 & 8.84 \\
\hline 5 & 2.2994 & 1.97 & 8.33 & 1.95 & 8.52 \\
\hline 10 & 2.3916 & 2.01 & 8.03 & 1.97 & 8.24 \\
\hline 15 & 2.4694 & 2.03 & 7.75 & 1.99 & 7.98 \\
\hline 20 & 2.5374 & 2.06 & 7.44 & 2.01 & 7.73 \\
\hline 24 & 2.5865 & 2.05 & 7.30 & 2.01 & 7.54 \\
\hline 73.2 & 3.0 & & & 2.10 & 5.48 \\
\hline 181.4 & 3.5 & & & 2.21 & 1.50 \\
\hline 195.9 & 3.55 & & & 2.22 & 0.74 \\
\hline 211.2 & 3.6 & & & - & - \\
\hline
\end{tabular}

TABLE II. OT-DFT zero temperature equation of state $P(\rho)$ of liquid ${ }^{4} \mathrm{He}$ and roton minimum parameters calculated by the method of Ref. 35. The experimental results are from Ref. 64 .

\section{Instability of the backflow term}

The dispersion relation of elementary excitations in liquid ${ }^{4} \mathrm{He}$ is shown in Fig 1. The low wavenumber $(q)$ region exhibits linear behaviour and corresponds to phonons (sound), followed by a maximum (maxon), and a high- $q$ region that corresponds to collective excitations called rotons. The latter region exhibits a distinct minimum around $q \approx 2 \AA^{-1}$ (roton minimum). Within the previously developed microscopic variational approach of Feynman and Cohen,, 63 a quantitative description of the roton minimum required the introduction of specific corrections to describe the correlated motion around each atom in the superfluid (backflow).

The formulation of the BF term in OT-DFT, 27 which is shown on the fourth line of Eq. (8), was inspired by a previous work of Thouless. ${ }^{65}$ With this term included, OT-DFT can accurately reproduce the $T=0$ experimental dispersion relation up to the solidification pressure ${ }^{64}$ with the exception of the turn-over region at high momenta beyond the roton region.

The roton minimum can be charaterised by two parameters, $\mu_{R}$ and $q_{R}$, by fitting the 
experimental dispersion relation close to the minimum with the following function at $T=0.5$ $\mathrm{K}^{64}$

$$
E(q)=\Delta+\frac{\hbar^{2}}{2 \mu_{R}}\left(q-q_{R}\right)^{2}
$$

where $q$ is the wavenumber, $\Delta$ is the roton energy, and $\mu_{R}$ defines the curvature at the roton minimum. A comparison of the experimental $\Delta$ and $q_{R}$ values with those obtained with the OT-DFT functional in shown in Table 【I.

The BF term becomes numerically unstable when $\rho \rightarrow 0$ and $|\mathbf{v}| \neq 0$. This instability is present in the energy functional as well as in the corresponding functional derivative yielding the effective potential in Eq. (8).66/67 Since the contribution of the BF term should be negligible at low densities, this problem can be eliminated by introducing a density cutoff for evaluating the velocity field from the probability current: $\mathbf{v}=\mathbf{j} /\left(\rho+\epsilon_{v}\right)$ where $\epsilon_{v}$ is the density cutoff value. Typical values of $\epsilon_{v}$ applied in recent work ${ }^{43}$ are in the order of $7 \times 10^{-5} \AA^{-3}$, which can be compared with the bulk liquid density $\rho_{0}=2.1836 \times 10^{-2} \AA^{-3}$ at $P=T=0$. An alternative approach is to neglect the BF term when the density becomes smaller than a given threshold value $\left(c a \cdot 10^{-6} \AA^{-3}\right)$ !

Figure 3 shows the density profile for a ${ }^{4} \mathrm{He}_{1000}$ droplet hosting a vortex line, which was calculated by the full OT-DFT or including only some of its term. The BF term reduces the vortex line energy $E_{V}$ by about $20 \mathrm{~K}$. Indeed, by using Eq. (30) and the definition of $E_{V}$ given in Sec. IIIB, one finds $127.1 \mathrm{~K}$ (basic OT-DFT); $124.2 \mathrm{~K}$ (KC OT-DFT); 107.5 (BF OT-DFT), and 105.5 K (OT-DFT).

With the sole exception of electrons, $\stackrel{41}{11}$ all attempts made so far to include the BF term in calculations modelling impurity dynamics in superfluid helium droplets or in bulk liquid have failed. One possible reason for such a failure is the appearance of a dynamic instability: the rationale for this being that the OT-DFT roton minimum energy collapses to zero around densities between 0.0355 and $0.036 \AA^{-3}$ as shown in Table II. Indeed, local liquid densities around impurities which exhibit strong binding towards helium may reach densities much higher than solid helium, leading to an unphysical behavior of the BF term and break down of the OT-DFT model.

It is likely that the BF term should only be applied in the liquid phase for which it was originally intended for. For example, the first solvation layers around snowball structures should be excluded from the BF interaction. A similar remark applies to the $\mathrm{KC}$ term, although it has not been found to be unstable. On the other hand, the solvation structures 
of electrons and vortices are free from such huge density pile-ups and the OT-DFT functional can be employed $\underline{41}$

The instability of the backflow term appearing at high densities calls for improvements. We present here a modified BF term that is numerically stable, only acts in the liquid phase and, by construction, yields a functional of the same quality as the original one ${ }^{27}$ in that physical region.

Consider a BF term of the following form

$$
\mathcal{H}_{B F}=-\frac{m_{4}}{4} \iint d \mathbf{r} d \mathbf{r}^{\prime} V_{J}\left(\left|\mathbf{r}-\mathbf{r}^{\prime}\right|\right) G(\rho(\mathbf{r})) G\left(\rho\left(\mathbf{r}^{\prime}\right)\right) \rho(\mathbf{r}) \rho\left(\mathbf{r}^{\prime}\right)\left[\mathbf{v}(\mathbf{r})-\mathbf{v}\left(\mathbf{r}^{\prime}\right)\right]^{2}
$$

If one takes

$$
G(\rho(\mathbf{r}))=\left[1-\Theta\left(\rho(\mathbf{r})-\rho_{B F}\right)\right]
$$

with $\rho_{B F}=0.033 \AA^{-3}$ and $\Theta(x)=1$ if $x \geq 0$ and zero otherwise, this will make the BF contribution effective only when helium is a 'true' liquid. The function $G(\rho(\mathbf{r}))$ is difficult to handle numerically. In practice, it has been substituted by

$$
G(\rho(\mathbf{r}))=\frac{1}{2}\left\{1-\tanh \left[\xi\left(\rho(\mathbf{r})-\rho_{B F}\right)\right]\right\}
$$

with a sufficiently large value of $\xi$ to make it steep at $\rho(\mathbf{r}) \sim \rho_{B F}$. Values used in the calculations are $\xi=10^{4} \AA^{3}$ and $\rho_{B F}=0.033 \AA^{-3}$.

The contribution of $\mathcal{H}_{B F}$ to the mean field, applied to the effective wave function $\Psi(\mathbf{r})$, is

$$
\begin{aligned}
& -\frac{m_{4}}{2}\left[\left.\rho(\mathbf{r}) \frac{d G}{d \rho}\right|_{\rho(\mathbf{r})}+G(\rho(\mathbf{r}))\right]\left\{\int d \mathbf{r}^{\prime} V_{J}\left(\left|\mathbf{r}-\mathbf{r}^{\prime}\right|\right) \rho\left(\mathbf{r}^{\prime}\right) G\left(\rho\left(\mathbf{r}^{\prime}\right)\right)\left[\mathbf{v}(\mathbf{r})-\mathbf{v}\left(\mathbf{r}^{\prime}\right)\right]^{2}\right\} \Psi(\mathbf{r}) \\
& +\frac{\imath \hbar}{2} \frac{1}{\rho(\mathbf{r})} \nabla_{\mathbf{r}} \cdot\left\{\int d \mathbf{r}^{\prime} V_{J}\left(\left|\mathbf{r}-\mathbf{r}^{\prime}\right|\right) \rho(\mathbf{r}) G(\rho(\mathbf{r})) \rho\left(\mathbf{r}^{\prime}\right) G\left(\rho\left(\mathbf{r}^{\prime}\right)\right)\left[\mathbf{v}(\mathbf{r})-\mathbf{v}\left(\mathbf{r}^{\prime}\right)\right]\right\} \Psi(\mathbf{r})
\end{aligned}
$$

Putting $G(\rho(\mathbf{r}))=1$ reduces it to the OT-DFT expression. ${ }^{6667}$ Eq. 19 is as complex to use as the original OT-DFT form. The modified OT-DFT functional (MOT-DFT), that includes the solid and modified BF terms, has been tested in dynamic calculations where OT-DFT was unstable; at variance, MOT-DFT has been found to be stable.

Having solved the instability problem in practice, let us mention that it is unclear what is the actual relevance of the $\mathrm{BF}$ term - and $\mathrm{KC}$ term - for most items addressed in this review. Processes such as photoexcitation and photoionisation of impurities usually involve high liquid densities and velocities in their first stages, which can lead to the production 
of shock waves, cavitation, and vorticity. None of them are very sensitive to the accurate description of the roton minimum and therefore the contribution of the BF term should be minimal (except for vorticity, for which we have already estimated the error if it is not included in the calculations). It is only after most of the excess energy has been dispersed into the fluid that the proper description of the elementary excitations becomes important, and the OT-DFT model can be applied at that point.

Last but not least, even if its systematic use might be computationally prohibitive, the stable MOT-DFT may be useful to carry out test calculations to calibrate simpler DFT approaches.

\section{TIME-INDEPENDENT CALCULATIONS}

In this section, we describe how to solve the time-independent EL equation, Eq. (7), to obtain the energetics and structure of solvated impurities in superfluid helium. For example, it can be used to determine absorption and emission spectra of atoms/molecules embedded in helium droplets. It also provides a starting point for subsequent time-dependent calculations.

\section{A. General considerations}

The ground state liquid density of a system can be obtained by solving Eq. (7). This is most often achieved by employing the imaginary time-method (ITM) ${ }^{[68}$ Normalization of the solution to a fixed number of helium atoms in the droplet, $N_{4}$, determines the corresponding chemical potential $\mu$. On the other hand, for the bulk liquid $\mu$ is dictated by the liquid equation of state, which can be obtained from $\mathcal{E}_{c}{ }^{69}$ Provided that a sufficiently large volume of liquid is considered, the value of the bulk chemical potential also applies to systems with solvated impurities or free surfaces.

Impurities much heavier than helium can be described classically as point-like particles, providing an external field for the helium density. In contrast, light impurities have to be modelled quantum mechanically based on the Schrödinger equation. In both cases, the impurity-He atom interaction, $V_{X}$, must be known: it is used to construct the impurity-liquid interaction using the pairwise sum approximation. For classical impurities, this interaction is included as an external field in the energy functional, $E[\rho]$, by integrating over the liquid 
density

$$
E[\rho] \rightarrow E[\rho]+\int d \mathbf{r} \rho(\mathbf{r}) V_{X}\left(\left|\mathbf{r}-\mathbf{r}_{I}\right|\right)
$$

where $\mathbf{r}_{I}$ is the location of the impurity. Eq. (7) is then written as

$$
\left\{-\frac{\hbar^{2}}{2 m_{4}} \nabla^{2}+\frac{\delta \mathcal{E}_{c}}{\delta \rho}+V_{X}\left(\left|\mathbf{r}-\mathbf{r}_{I}\right|\right)\right\} \Psi(\mathbf{r})=\mu \Psi(\mathbf{r})
$$

For impurities requiring quantum mechanical treatment, $E[\rho]$ must also take into account their zero point motion

$$
E[\rho] \rightarrow E[\rho]+\frac{\hbar^{2}}{2 m_{I}} \int d \mathbf{r}_{I}\left|\nabla_{I} \phi\left(\mathbf{r}_{I}\right)\right|^{2}+\iint d \mathbf{r} d \mathbf{r}_{I} \rho(\mathbf{r}) V_{X}\left(\left|\mathbf{r}-\mathbf{r}_{I}\right|\right)\left|\phi\left(\mathbf{r}_{I}\right)\right|^{2}
$$

where $\phi\left(\mathbf{r}_{I}\right)$ is the impurity wave function and $m_{I}$ its mass. This yields two coupled equations, one for the liquid and another for the impurity

$$
\begin{array}{r}
\left\{-\frac{\hbar^{2}}{2 m_{4}} \nabla^{2}+\frac{\delta \mathcal{E}_{c}}{\delta \rho}+\int d \mathbf{r}_{I} V_{X}\left(\left|\mathbf{r}-\mathbf{r}_{I}\right|\right)\left|\phi\left(\mathbf{r}_{I}\right)\right|^{2}\right\} \Psi(\mathbf{r})=\mu \Psi(\mathbf{r}) \\
\left\{-\frac{\hbar^{2}}{2 m_{I}} \nabla_{I}^{2}+\int d \mathbf{r} V_{X}\left(\left|\mathbf{r}-\mathbf{r}_{I}\right|\right) \rho(\mathbf{r})\right\} \phi\left(\mathbf{r}_{I}\right)=\varepsilon \phi\left(\mathbf{r}_{I}\right)
\end{array}
$$

In some applications, it may be necessary to fix the distance between the impurity and the centre of mass (COM) of the droplet. This is the case, e.g., when calculating the energy as a function of that distance allows to determine possible energy barriers hindering the motion of the impurity. This can be achieved by including a constraint term in the energy functional. Assuming that the classical impurity lies along the $z$-axis, the constraint can be introduced as

$$
E[\rho]+\int d \mathbf{r} \rho(\mathbf{r}) V_{X}\left(\left|\mathbf{r}-\mathbf{r}_{I}\right|\right)+\frac{\lambda_{C}}{2}\left(\mathcal{Z}-\mathcal{Z}_{0}\right)^{2}
$$

where $\mathcal{Z}$ is the instantaneous distance between the impurity and the COM of the droplet, $\mathcal{Z}_{0}$ is the corresponding preset constrained distance, and $\lambda_{C}$ is a constant determining the strength of the penalty term. Typical values of $\lambda_{C}$ to ensure that the desired distance is retained within $0.1 \%$ accuracy are in the $1000-3000 \mathrm{~K}^{-2}$ range.

To illustrate how this constraint influences the EL equations when the impurity is treated as a quantum particle, we first define the droplet COM position and the expectation value for the quantum impurity position along the $z$-axis

$$
\begin{aligned}
Z_{C M} & =\frac{1}{N_{4}} \int d \mathbf{r} z \rho(\mathbf{r}) \\
z_{i m p} & =\int d \mathbf{r}_{I} z_{I}\left|\phi\left(\mathbf{r}_{I}\right)\right|^{2}
\end{aligned}
$$


With these definitions, Eqs 23) become

$$
\begin{aligned}
& \left\{-\frac{\hbar^{2}}{2 m_{4}} \nabla^{2}+\frac{\delta \mathcal{E}_{c}}{\delta \rho}+\int d \mathbf{r}_{I} V_{X}\left(\left|\mathbf{r}-\mathbf{r}_{I}\right|\right)\left|\phi\left(\mathbf{r}_{I}\right)\right|^{2}+\lambda_{C}\left[\mathcal{Z}-\mathcal{Z}_{0}\right]\left(\frac{-z}{N_{4}}\right)\right\} \Psi(\mathbf{r})=\mu \Psi(\mathbf{r}) \\
& \left\{-\frac{\hbar^{2}}{2 m_{I}} \nabla_{I}^{2}+\int d \mathbf{r} V_{X}\left(\left|\mathbf{r}-\mathbf{r}_{I}\right|\right) \rho(\mathbf{r})+\lambda_{C}\left[\mathcal{Z}-\mathcal{Z}_{0}\right] z_{I}\right\} \phi\left(\mathbf{r}_{I}\right)=\varepsilon \phi\left(\mathbf{r}_{I}\right)
\end{aligned}
$$

where $\mathcal{Z}=z_{i m p}-Z_{C M}$. For a classical impurity, the term $\lambda_{C}\left[\mathcal{Z}-\mathcal{Z}_{0}\right]\left(-z / N_{4}\right)$ has to be added to the left hand side of Eq. (21); in this case $z_{i m p}$ is the impurity position.

The DFT equations (21) or 23) can be solved by the ITM in cartesian coordinates. 68 Most calculations are carried out in full 3D without taking advantage of possible symmetries in the external potential. Densities, wave functions, differential operators, etc., are represented on discrete equally spaced cartesian grids. The spatial step employed in these calculations is typically ca. $0.4 \AA$. The differential operators (first and second derivatives) are represented by $k$-point formulas or evaluated directly in the Fourier space using the split operator technique. ${ }^{67}$ In the former case, 13 -point formulas have been found accurate enough.

Since the integral terms in OT-DFT can be expressed as convolutions, 2 27]60|67 they can be conveniently computed in the Fourier space. Therefore, a key tool for an efficient numerical implementation of OT-DFT is the Fast Fourier Transformation (FFT) technique ${ }^{70}$ FFT algorithms are well established in the literature and have efficient parallel implementations. Note that many of the transformations required for evaluating the OT-DFT functional need to be carried out only once.

\section{B. Introduction of vorticity}

In order to represent a sustained current in liquid helium, the order parameter must be a complex valued function. This is the case for a vortex line, which involves liquid circulation around its core. Vorticity around the symmetry axis $(z)$ of an axially symmetric helium droplet can be represented by

$$
\Psi(r, \theta) \equiv \rho^{1 / 2}(r) e^{\imath m \theta}
$$

where $r$ is the distance from the symmetry axis, $\theta$ the polar angle, and $m$ the circulation quantum number. This is an eigenfunction of the total angular momentum operator, $\hat{L}_{z} \Psi(r, \theta)=m N_{4} \hbar \Psi(r, \theta)$. In practice, only configurations with circulation $m= \pm 1$ are relevant. This is because the kinetic energy of a vortex line is proportional to $m^{2}$ and therefore 
a vortex line with $m= \pm 2$ is energetically less favored than two separate vortex lines with $m= \pm 1$. A single vortex line in a pure ${ }^{4} \mathrm{He}_{500}$ droplet, in a mixed ${ }^{4} \mathrm{He}_{500}+{ }^{3} \mathrm{He}_{100}$ droplet, and the same systems doped with an HCN molecule can be seen in Fig. 30 of Ref. 7.

The EL equations are as for vortex-free droplets, Eqs. (6) and (21), but the effective wave function has to be complex valued. Since the ITM can only converge to a solution that has overlap with the initial order parameter, starting the calculation with an initial guess similar to Eq. 27) will automatically yield the vortex solution. For instance, a vortex line along the $z$ axis can be produced by starting the imaginary-time calculation with the following initial order parameter

$$
\Psi(\mathbf{r})=\frac{\rho_{0}^{1 / 2}(\mathbf{r})}{\sqrt{x^{2}+y^{2}}}(x+\imath y)
$$

where $\rho_{0}(\mathbf{r})$ is the density corresponding to either a pure or doped droplet without vortex. In cylindrical coordinates, this expression reduces to Eq. (27) with $m=1$ provided that the density is axially symmetric. For a more detailed discussion, see e.g. Ref. 69.

The energetics of pure and doped helium hosting vortices are usually characterised by the following quantities $43169171 / 72$

- Solvation energy of the impurity $X: S_{X}=E\left(X @{ }^{4} \mathrm{He}_{N}\right)-E\left({ }^{4} \mathrm{He}_{N}\right)$

- Vortex energy: $E_{V}=E\left(V @{ }^{4} \mathrm{He}_{N}\right)-E\left({ }^{4} \mathrm{He}_{N}\right)$

- Binding energy of the impurity $X$ to the vortex:

$$
B_{X}=S_{X}-\left\{E\left[(X+V) @{ }^{4} \mathrm{He}_{N}\right]-E\left(V @{ }^{4} \mathrm{He}_{N}\right)\right\}
$$

The binding energy is the result of a delicate balance between the contributing terms and the resulting values are typically rather small. For example, the binding energy of a Xe

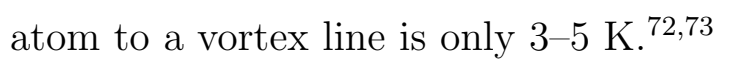

The kinetic energy of the superfluid flow in the volume excluded by the impurity intuitively corresponds to $B_{X}$ and for this reason it is also called 'substitution energy'. ${ }^{74}$ Using a classical sharp wall model for the impurity bubble and vortex line, the binding energy can be approximated as

$$
B_{X}=2 \pi \frac{\hbar^{2}}{m_{4}} \rho_{0} R_{X}\left\{\left(1+\frac{a^{2}}{R_{X}^{2}}\right)^{1 / 2} \ln \left[\frac{R_{X}}{a}+\sqrt{\left(\frac{R_{X}}{a}\right)^{2}+1}\right]-1\right\}
$$

where $a$ is the radius of the vortex core and $R_{X}$ the radius of the atomic bubble. Using the Xe atom as an example, setting the liquid density to the $T=P=0$ value $\rho_{0}=0.0218$ 
$\AA^{-3}, a=1 \AA$, and the bubble radius to the value where the Xe-He pair potential becomes repulsive, $R_{X}=3.5 \AA$, Eq. 29 yields a binding energy $B_{X}=6.1 \mathrm{~K}$.

The critical angular velocity for nucleating the vortex line represented by Eqs. (27) or (28) in a droplet consisting of $N_{4}$ helium atoms is given by ${ }^{75}$

$$
\omega_{c}=\frac{1}{\hbar} \frac{E_{V}}{N_{4}}
$$

where $E_{V}$ is the vortex energy as defined above. For a ${ }^{4} \mathrm{He}_{1000}$ droplet this gives $\omega_{c}=$ $0.127 \mathrm{~K} / \hbar=0.0166 \mathrm{ps}^{-1}$.

The above approach can be used to create individual vortex lines. A different strategy has to be employed to generate an array of vortex lines. A rotational constraint is imposed in the rotating frame of reference ('co-rotating frame') by solving the following EL equation

$$
\left[\mathcal{H}-\omega \hat{L}_{z}\right] \Psi(\mathbf{r})=\mu \Psi(\mathbf{r}),
$$

where $\mathcal{H}$ is the DFT Hamiltonian (Eq. (6)), $\hat{L}_{z}$ is the $z$-component of the angular momentum operator, and $\omega$ is the angular velocity of the co-rotating frame.

Note that for a vortex array $\Psi(\mathbf{r})$ is no longer an eigenvector of the angular momentum.

The initial guess for imaginary-time evolution can be obtained by the 'imprinting' method; for $n_{v}$ vortex lines, the initial guess $\Psi(\mathbf{r})$ is written as

$$
\Psi(\mathbf{r})=\rho_{0}^{1 / 2}(\mathbf{r}) \prod_{j=1}^{n_{v}}\left[\frac{\left(x-x_{j}\right)+\imath\left(y-y_{j}\right)}{\sqrt{\left(x-x_{j}\right)^{2}+\left(y-y_{j}\right)^{2}}}\right]
$$

where $\rho_{0}(\mathbf{r})$ is the density of the vortex-free droplet and $\left(x_{j}, y_{j}\right)$ is the initial position of the $j^{\text {th }}$ linear vortex core parallel to the $z$-axis. Note that the expression for $\Psi(\mathbf{r})$ was incorrectly written in Refs. 71 and 73. During the imaginary-time relaxation, the positions of the vortex lines will change until convergence to the lowest energy configuration for a given $\omega$ is reached. Complex configurations hosting several vortex lines (vortex arrays) will be described in Sec. VM.

\section{DYNAMICS}

Given a static initial configuration $\Psi(\mathbf{r})$ and a known additional perturbation to drive the system, its dynamic evolution can be followed in real-time. The additional perturbation can be, for instance, a sudden photoionisation or photoexcitation of the impurity. As discussed 
above, a classical or quantum description is employed to propagate the impurity degrees of freedom, depending on its mass as compared to a helium atom.

\section{A. Heavy impurities}

Heavy impurities with no evolution in their electronic degrees of freedom can be treated using classical mechanics. Examples include photoexcitation of heavy alkali metal atoms (e.g. $\mathrm{Rb}, \mathrm{Cs})$ from the $n$ s electronic ground state to the $(n+1)$ s excited state ${ }^{76}$ and photoionisation of a Ba atom ${ }^{77}$ (see also Ref. 32) in helium droplets. Typically, these photoexcitation and photoionisation processes are considered to be instantaneous, which means that the light pulse is short enough that the nuclei do not have time to move, but is long enough that its energy spread covers only one (excited or ionised) electronic state.

After ionisation or electronic excitation, the total energy of the system is written as

$$
E\left[\Psi, \mathbf{r}_{I}\right]=\int d \mathbf{r} \frac{\hbar^{2}}{2 m_{4}}|\nabla \Psi|^{2}+\frac{p_{I}^{2}}{2 m_{I}}+\int d \mathbf{r} \mathcal{E}_{c}(\rho)+\int d \mathbf{r} \rho(\mathbf{r}) V_{X^{*}}\left(\left|\mathbf{r}-\mathbf{r}_{I}\right|\right)
$$

where $I$ denotes the impurity and $V_{X^{*}}$ is the $X$-He pair potential for the excited or ionised state. $V_{X^{*}}$ (and $V_{X}$ in Sec. III A) are usually obtained from high-level ab initio calculations ${ }^{78}$ or accurate semi-empirical methods. Since helium mostly interacts with other species through weak van der Waals forces, accurate treatment of electron correlation is very important.

The time evolution of the helium order parameter $\Psi(\mathbf{r}, t)$ and the impurity position $r_{I}(t)$ can be obtained from the TDDFT and Newton equations, respectively

$$
\begin{aligned}
i \hbar \frac{\partial}{\partial t} \Psi & =\left[-\frac{\hbar^{2}}{2 m_{4}} \nabla^{2}+\frac{\delta \mathcal{E}_{c}}{\delta \rho}+V_{X^{*}}\left(\left|\mathbf{r}-\mathbf{r}_{I}\right|\right)\right] \Psi \\
m_{I} \ddot{\mathbf{r}}_{I} & =-\nabla_{\mathbf{r}_{I}}\left[\int d \mathbf{r} \rho(\mathbf{r}) V_{X^{*}}\left(\left|\mathbf{r}-\mathbf{r}_{I}\right|\right)\right]=-\int d \mathbf{r} V_{X^{*}}\left(\left|\mathbf{r}-\mathbf{r}_{I}\right|\right) \nabla \rho(\mathbf{r})
\end{aligned}
$$

For a light impurity (i.e. quantum mechanical treatment), Eqs. (33) and (34) become

$$
\begin{aligned}
E[\Psi, \phi] & =\int d \mathbf{r} \frac{\hbar^{2}}{2 m_{4}}|\nabla \Psi|^{2}+\int d \mathbf{r}_{I} \frac{\hbar^{2}}{2 m_{I}}\left|\nabla_{\mathbf{r}_{I}} \phi\right|^{2}+\int d \mathbf{r} \mathcal{E}_{c}(\rho) \\
& +\iint d \mathbf{r} d \mathbf{r}_{I} \rho(\mathbf{r}, t) V_{X^{*}}\left(\left|\mathbf{r}-\mathbf{r}_{I}\right|\right)\left|\phi\left(\mathbf{r}_{I}, t\right)\right|^{2}
\end{aligned}
$$


and

$$
\begin{aligned}
& i \hbar \frac{\partial}{\partial t} \Psi(\mathbf{r}, t)=\left[-\frac{\hbar^{2}}{2 m_{4}} \nabla^{2}+\frac{\delta \mathcal{E}_{c}}{\delta \rho}+\int d \mathbf{r}_{I} V_{X^{*}}\left(\left|\mathbf{r}-\mathbf{r}_{I}\right|\right)\left|\phi\left(\mathbf{r}_{I}, t\right)\right|^{2}\right] \Psi(\mathbf{r}, t) \\
& i \hbar \frac{\partial}{\partial t} \phi\left(\mathbf{r}_{I}, t\right)=\left[-\frac{\hbar^{2}}{2 m_{I}} \nabla_{I}^{2}+\int d \mathbf{r} V_{X^{*}}\left(\left|\mathbf{r}-\mathbf{r}_{I}\right|\right) \rho(\mathbf{r}, t)\right] \phi\left(\mathbf{r}_{I}, t\right)
\end{aligned}
$$

where $\phi\left(\mathbf{r}_{I}, t\right)$ is the wave function for the impurity. Since the dynamics of the impurity and that of the liquid tend to have very different time scales, the overall time step has to be chosen with care. A safe choice of the shorter one for both equations can, however, increase the computational time significantly. Using sub-steps for the faster component can in part alleviate such issues. Another problem can arise from the spatial grids. Unless interpolation techniques are employed, both the impurity and the liquid grids must have the same size and step length. Since light impurities are usually fast and therefore require fine grids, this also increases the computational time required for the liquid. An elegant way out of this problem is to propagate the impurity using the so-called 'test particle' method ${ }^{79}$ This approach has been used to simulate the $\mathrm{Na}$ and Li atom dynamics in helium droplets following the $(n+1) \mathrm{s}$ $\leftarrow n$ s excitation. 80

A more complicated situation is encountered when the impurity electronic degrees of freedom must also be included in the dynamics. For example, when an impurity is excited from a spherical $n$ s to a $n^{\prime} \mathrm{p}$ state, the three degenerate $\mathrm{p}$ states are split by the dynamic Jahn-Teller effect. The interaction between a He atom and the $L=1$ state impurity can be decomposed into $\Sigma(\Lambda=0)$ and a doubly degenerate $\Pi(\Lambda= \pm 1)$ state, where $\Lambda$ is the projection of the orbital angular momentum on the interatomic axis. So far, only the case where the impurity can be treated classically has been considered $\stackrel{44}{ }$ To account for the dynamic orientation of the p-orbital, a simple diatomics-in-molecules (DIM) model can be applied $[183$ Its basic ingredients are given below.

The electronic structure of a $n^{\prime}$ p-state impurity (i.e. effective one-electron excited ${ }^{2} \mathrm{P}$ atomic state) interacting with He atoms can be expressed in an effective one-electron porbital basis. In the diatomic frame coinciding with the $n^{\text {th }}$ helium atom $\left({ }^{1} \mathrm{~S}\right)$ along the $z_{n}$-axis, the minimal DIM basis set is $\left|p_{x n}\right\rangle,\left|p_{y n}\right\rangle,\left|p_{z n}\right\rangle$, and the helium-impurity interaction is given by

$$
U\left(r_{n}\right)=V_{\Pi}\left(r_{n}\right) \mathbf{I}+\left\{V_{\Sigma}\left(r_{n}\right)-V_{\Pi}\left(r_{n}\right)\right\}\left|p_{z n}\right\rangle\left\langle p_{z n}\right|
$$

where $r_{n}$ is the interatomic distance and $V_{\Pi}(r)$ and $V_{\Sigma}(r)$ are the $\Pi$ and $\Sigma$ impurity-He pair potentials in the absence of spin-orbit coupling. 
For a system consisting of $N_{4}$ helium atoms and an excited p-state impurity, the total potential energy is constructed using the DIM model 81

$$
U=\sum_{n=1}^{N_{4}}\left\{V_{\Pi}\left(r_{n}\right) \mathbf{I}+\left[V_{\Sigma}\left(r_{n}\right)-V_{\Pi}\left(r_{n}\right)\right] R_{n}\left|p_{z}\right\rangle\left\langle p_{z}\right| R_{n}^{-1}\right\}
$$

where $R_{n}$ is a rotation matrix which transforms the common laboratory frame to the diatomic frame corresponding to the $n^{\text {th }}$ He atom. In cartesian coordinates

$$
\left\langle p_{i}\left|R_{n}\right| p_{z}\right\rangle\left\langle p_{z}\left|R_{n}^{-1}\right| p_{j}\right\rangle=\frac{r_{i n} r_{j n}}{r_{n}^{2}}
$$

where $r_{1 n} \equiv x_{n}, r_{2 n} \equiv y_{n}, r_{3 n} \equiv z_{n}$, and $r_{n}^{2}=x_{n}^{2}+y_{n}^{2}+z_{n}^{2}$ for the $n^{\text {th }}$ He atom. The matrix elements of the DIM Hamiltonian are then

$$
\left\langle p_{i}|U| p_{j}\right\rangle \equiv U_{i j}=\sum_{n=1}^{N_{4}}\left\{V_{\Pi}\left(r_{n}\right) \delta_{i j}+\left[V_{\Sigma}\left(r_{n}\right)-V_{\Pi}\left(r_{n}\right)\right] \frac{r_{i n} r_{j n}}{r_{n}^{2}}\right\}
$$

Since DFT provides a continuous distribution, the discrete sum over helium atoms is replaced by integration over the density $\left(\sum_{n} \rightarrow \int \mathrm{d}^{3} \mathbf{r}^{\prime \prime} \rho\left(\mathbf{r}^{\prime \prime}\right)\right)$, which gives

$$
U_{i j}(\mathbf{r})=\int \mathrm{d}^{3} \mathbf{r}^{\prime} \rho\left(\mathbf{r}^{\prime}+\mathbf{r}\right)\left\{V_{\Pi}\left(r^{\prime}\right) \delta_{i j}+\left[V_{\Sigma}\left(r^{\prime}\right)-V_{\Pi}\left(r^{\prime}\right)\right] \frac{r_{i}^{\prime} r_{j}^{\prime}}{r^{\prime 2}}\right\}
$$

The eigenvalues $V_{m}^{\text {ex }}(\mathbf{r})$ of this real symmetric matrix define the potential energy curves (PEC) as a function of the distance between the surrounding helium and the impurity.

The above model assumes that spin-orbit (SO) coupling is negligible. However, when it becomes comparable to the helium induced splitting of the p-orbitals, it must be included in the calculation. The total Hamiltonian is then given by $U_{T}=U+U_{S O}$ where $U_{S O}$ is the SO hamiltonian matrix, usually approximated by that of the free atom. ${ }^{84}$ The previously mentioned minimal DIM basis set can be extended to include the electron spin: $s=\uparrow\left(m_{s}=\right.$ $1 / 2), s=\downarrow\left(m_{s}=-1 / 2\right)$, i.e. $|i, s\rangle \equiv\left|p_{x}, \uparrow\right\rangle,\left|p_{x}, \downarrow\right\rangle,\left|p_{y}, \uparrow\right\rangle,\left|p_{y}, \downarrow\right\rangle,\left|p_{z}, \uparrow\right\rangle,\left|p_{z}, \downarrow\right\rangle$.

Kramers' theorem states that the two-fold degeneracy of the levels originating from total half-integer spin cannot be broken by electrostatic interactions. ${ }^{85}$ Thus, all the electronic eigenstates of $U_{T}$ are doubly degenerate. Diagonalization of $U_{T}$ yields three doubly degenerate PEC between the impurity and surrounding helium. This method has also been extended to impurities in D electronic states $\underline{86187}$

The DIM wave function of the impurity, $|\lambda\rangle$, is determined by a six-dimensional state vector

$$
|\lambda\rangle=\sum_{\substack{i=x, y, z \\ s=-1 / 2,1 / 2}} \lambda_{i s}|i, s\rangle
$$


The complete set of variables required to describe the system consists of the complex valued effective wave function for helium $\Psi(\mathbf{r}, t)$ with $\rho(\mathbf{r}, t)=|\Psi(\mathbf{r}, t)|^{2}$, the impurity position $\mathbf{r}_{I}(t)$, and the 6-dimensional complex vector to determine its electronic wave function $|\lambda(t)\rangle$. The total energy of the impurity- ${ }^{4} \mathrm{He}_{N}$ complex after excitation to the ${ }^{2} \mathrm{P}$ manifold is

$$
E\left[\Psi, \mathbf{r}_{I}, \lambda\right]=\int d \mathbf{r} \frac{\hbar^{2}}{2 m}|\nabla \Psi|^{2}+\frac{p_{I}^{2}}{2 m_{I}}+\int d \mathbf{r} \mathcal{E}_{c}[\rho]+\left\langle\lambda\left|V_{S O}\right| \lambda\right\rangle+\int d \mathbf{r} \rho(\mathbf{r}) V_{\lambda}\left(\mathbf{r}-\mathbf{r}_{I}\right)
$$

where $V_{S O}$ is the spin-orbit coupling operator and $V_{\lambda}$ is defined as

$$
V_{\lambda}(\mathbf{r}) \equiv\langle\lambda|\mathcal{V}(\mathbf{r})| \lambda\rangle=\sum_{i j s s^{\prime}} \lambda_{i s}^{*} \mathcal{V}^{i j s s^{\prime}}(\mathbf{r}) \lambda_{j s^{\prime}}
$$

with the components of the six-dimensional matrix $\mathcal{V}$ given by

$$
\mathcal{V}^{i j s s^{\prime}}(\mathbf{r})=\left[V_{\Pi}(r) \delta_{i j}+\left(V_{\Sigma}(r)-V_{\Pi}(r)\right) \frac{r_{i} r_{j}}{r^{2}}\right] \delta_{s s^{\prime}}
$$

The time evolution of the system is obtained by minimizing the action

$$
\mathcal{A}\left[\Psi, \mathbf{r}_{I}, \lambda\right]=\int d t\left\{E\left[\Psi, \mathbf{r}_{I}, \lambda\right]-i \hbar \int d \mathbf{r} \Psi^{*}(\mathbf{r}) \frac{\partial}{\partial t} \Psi(\mathbf{r})-i \hbar\left\langle\lambda\left|\frac{\partial}{\partial t}\right| \lambda\right\rangle-\frac{1}{2} m_{I} \dot{\mathbf{r}}_{I}^{2}\right\}
$$

Variation of $\mathcal{A}$ with respect to $\Psi^{*},\langle\lambda|$ and $\mathbf{r}_{I}$ yields

$$
\begin{aligned}
& i \hbar \frac{\partial}{\partial t} \Psi=\left[-\frac{\hbar^{2}}{2 m} \nabla^{2}+\frac{\delta \mathcal{E}_{c}}{\delta \rho(\mathbf{r})}+V_{\lambda}\left(\mathbf{r}-\mathbf{r}_{I}\right)\right] \Psi \\
& i \hbar \frac{\partial}{\partial t}|\lambda\rangle=\mathcal{H}|\lambda\rangle \\
& m_{I} \ddot{\mathbf{r}}_{I}=-\nabla_{\mathbf{r}_{I}}\left[\int d \mathbf{r} \rho(\mathbf{r}) V_{\lambda}\left(\mathbf{r}-\mathbf{r}_{I}\right)\right]=-\int d \mathbf{r} V_{\lambda}\left(\mathbf{r}-\mathbf{r}_{I}\right) \nabla \rho(\mathbf{r})
\end{aligned}
$$

where the explicit time dependence of the variables is omitted for clarity. The second line of Eq. 47 is a $6 \times 6$ matrix equation with the matrix elements given by

$$
H^{i j s s^{\prime}}=\int d \mathbf{r} \rho(\mathbf{r}) \mathcal{V}^{i j s s^{\prime}}\left(\mathbf{r}-\mathbf{r}_{I}\right)+V_{S O}^{i j s s^{\prime}}
$$

In order to solve Eqs. (34), (36) or (47), initial values for the variables must be specified. Their choice is guided by the physics of the process studied. The initial helium order parameter and the initial impurity position are usually taken from the static solution of the doped droplet, with the initial impurity velocity set to zero. The initial choice for $|\lambda\rangle$ is dictated by the optical excitation process. It is often taken as one of the eigenstates of the DIM hamiltonian at the time of the electronic excitation. 
All dynamic equations in this Section, as e.g. Eq (47), have been solved by using Hamming's predictor-modifier-corrector method,, 88 initiated by a fourth-order Runge-Kutta-Gill algorithm. $.88 / 89$ The integration time step employed in most applications is about 0.5 fs.

The time-dependent relaxation of liquid helium around excited state impurities leads to the creation of sound waves and even shock waves when steep repulsive interactions are present. In helium droplets this can also lead to helium evaporation at the droplet surface. Eventually, evaporated helium and bulk liquid excitations will reach the simulation box boundaries and re-enter the box from the opposite side [periodic boundary conditions (PBC) are implied by the use of FFT to compute the convolution integrals in the OT-DFT equations]. This can interfere with the system in an unphysical and unpredictable way, and lead to significant errors in the calculations.

To avoid such artifacts, absorbing boundaries should be implemented by replacing $\imath \longrightarrow$ $\imath+\Lambda(\mathbf{r})$ in the time-dependent OT-DFT equation. $\stackrel{46}{ }$ The attenuation field $\Lambda(\mathbf{r})$ has the form

$$
\Lambda(\mathbf{r})=\Lambda_{0}\left[1+\tanh \left(\frac{s-s_{0}}{a}\right)\right], \quad s \equiv|\mathbf{r}|
$$

No attenuation takes place when $s<\left(s_{0}-2 a\right)$ since $\Lambda(\mathbf{r}) \ll 1$. The absorbing region has to be large enough to remove all the unwanted effects due to the presence of the PBC. Note that for this method to work for bulk helium, the chemical potential must be included in the external potential during the TDDFT evolution. $\underline{46}$

Finally, we mention that exciplex configurations can also be studied by DFT. The method, which was inspired by the molecular model of Ref. 90, is discussed in detail in Ref. 86.

\section{B. Test particle method for light impurities}

If the impurity-helium interaction is highly repulsive in the impurity excited state, its velocity can quickly become very large. Inside the droplet this velocity tends to fall below the Landau critical velocity because the kinetic energy is dissipated through efficient coupling to

elementary excitations of the liquid. This process is not instantaneous ${ }^{7791}$ and the impurity velocity can remain high during this initial period. Furthermore, in the case of helium droplets velocities may remain high indefinitely if the impurity leaves it. The wave packet for a light impurity with high velocity exhibits rapid spatial and temporal oscillations, which require the use of very fine spatial grids and short time steps. Since these grids must be 
compatible with the ones used for helium, the computation especially in 3D becomes quickly unaffordable.

To avoid this problem, the impurity degrees of freedom can be described by Bohmian dynamics ${ }^{[79}$ This approach, which is equivalent to solving the Schrödinger equation, has been tested for the dynamics of excited state $\mathrm{Li}$ and $\mathrm{Na}$ atoms ejected from the helium droplet surface ${ }^{80}$ An overview of this method is given below.

The second line in Eq. (36) can effectively be cast into the format of a time-dependent Schrödinger equation (note that the $I$ index to $\mathbf{r}_{I}$ is dropped to simplify the notation)

$$
\imath \hbar \frac{\partial}{\partial t} \phi(\mathbf{r}, t)=\left[-\frac{\hbar^{2}}{2 m} \nabla^{2}+V(\mathbf{r})\right] \phi(\mathbf{r}, t) .
$$

Using the hydrodynamic form suggested by Madelung, $\frac{40}{2}$ the complex wave function can be written as

$$
\phi(\mathbf{r}, t) \equiv \chi(\mathbf{r}, t) e^{i \mathcal{S}(\mathbf{r}, t)}
$$

where $\chi \geq 0$ and $\mathcal{S}$ are both real valued functions. ${ }^{79}$ While the real and imaginary parts of $\phi$ may oscillate rapidly, the behavior of $\chi$ and $\mathcal{S}$ is much smoother than $\phi$ as a function of time. ${ }^{80}$ The associated velocity field and the current density are defined as $\mathbf{v}(\mathbf{r}, t) \equiv$ $(\hbar / m) \nabla \mathcal{S}$ and $\mathbf{j}(\mathbf{r}, t) \equiv \hbar /(2 m i)\left[\phi^{*} \nabla \phi-\phi \nabla \phi^{*}\right]=\chi^{2} \mathbf{v}$. Substitution of Eq. (51) into Eq. (50) and equating the real and imaginary parts of the left and right hand side terms in Eq. (50) yields the following - quantum hydrodynamic - equations for $\chi$ and $\mathcal{S}$ :

$$
\begin{aligned}
\frac{\partial \chi^{2}}{\partial t} & =-\nabla \cdot \mathbf{j} & & \text { [continuity equation] } \\
-\hbar \frac{\partial \mathcal{S}}{\partial t} & =\frac{1}{2} m \mathbf{v}^{2}+\mathcal{Q}(\mathbf{r}, t)+V(\mathbf{r}) & & \text { [quantum Hamilton - Jacobi equation] }
\end{aligned}
$$

where $\mathcal{Q}$ is the so-called quantum potential (or quantum pressure)

$$
\mathcal{Q}(\mathbf{r}, t) \equiv-\frac{\hbar^{2}}{2 m} \frac{\nabla^{2} \chi}{\chi}
$$

The above Eq. (52) can be solved by using the test particle method as follows. The probability density $\chi^{2}$ and the current density as a function of time can be constructed from a histogram based on $M$ test particles. Given a set of test particle trajectories, $\left\{\mathbf{R}_{i}(t)\right\}_{i=1}^{M}$, where $\mathbf{R}_{i}(t)=\mathbf{R}\left(\mathbf{r}_{i}, t\right)$ and $\mathbf{R}_{i}(0)=\mathbf{r}_{i}, \chi^{2}$ and $\mathbf{j}$ can be computed as

$$
\begin{aligned}
\chi^{2}(\mathbf{r}, t) & =\lim _{M \rightarrow \infty} \frac{1}{M} \sum_{i=1}^{M} \delta\left[\mathbf{r}-\mathbf{R}_{i}(t)\right] \\
\mathbf{j}(\mathbf{r}, t) & =\lim _{M \rightarrow \infty} \frac{1}{M} \sum_{i=1}^{M} \mathbf{v}\left[\mathbf{R}_{i}(t)\right] \delta\left[\mathbf{r}-\mathbf{R}_{i}(t)\right] .
\end{aligned}
$$




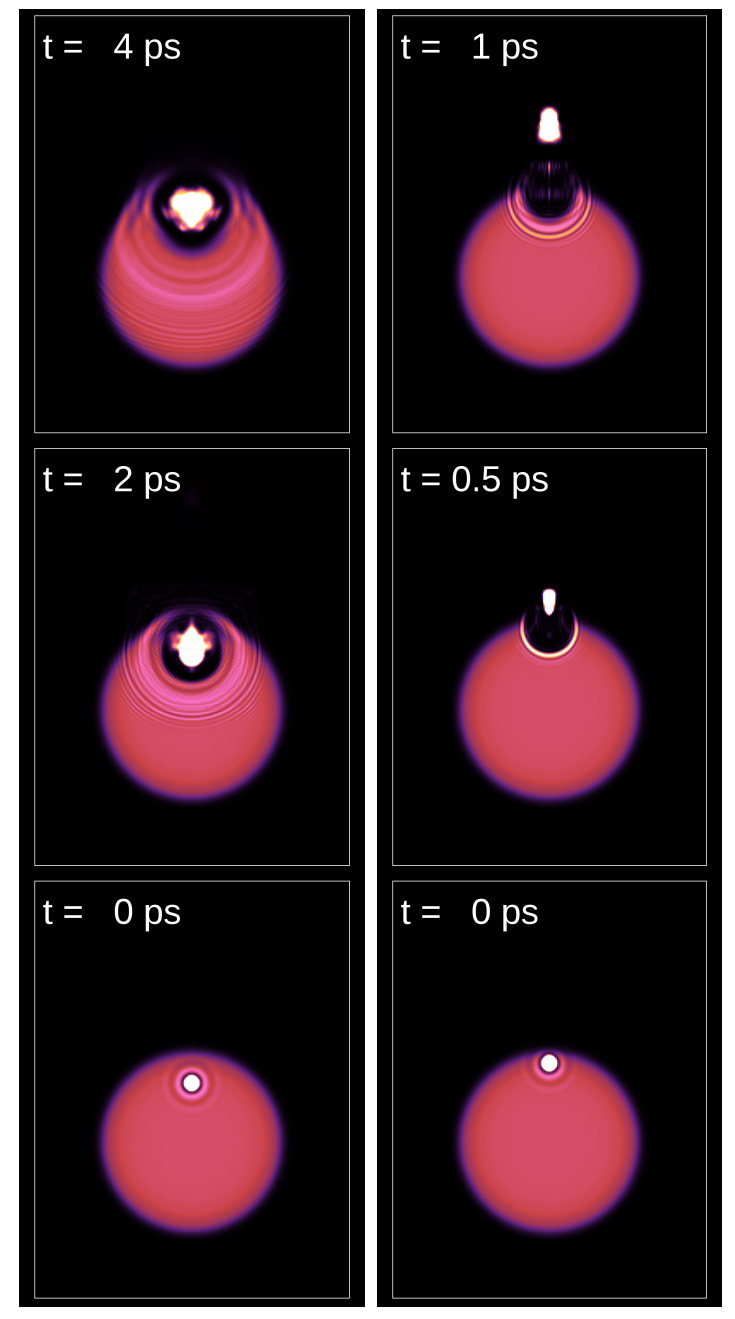

FIG. 4. (a) Snapshots of the He density during the evolution of the $\mathrm{He}^{*} @{ }^{4} \mathrm{He}_{1000}$ starting from $\mathbf{r}_{I}=15 \AA$. The bright yellow spot is the probability distribution of the He* being ejected. (b) Same as (a) starting from $\mathbf{r}_{I}=20 \AA$.

For example, a value of $M=200000$ was used in Ref. 80 to simulate the desorption of Li and $\mathrm{Na}$ atoms excited to the $3 \mathrm{~s}$ and 4 s states, respectively.

The continuity equation is automatically fulfilled provided that $\dot{\mathbf{R}}_{i}(t)=\mathbf{v}\left[\mathbf{R}_{i}(t)\right]$, i.e. the test particle velocity must be equal to the value of the velocity field at that point. By taking the gradient of both sides of the second line in Eq. (52) and rewriting it in the Lagrangian reference frame $(d / d t=\partial / \partial t+\mathbf{v} \cdot \nabla)$, the following equation of motion for the test particles 
is obtained ('Quantum Newton equation')

$$
m \ddot{\mathbf{R}}_{i}(t)=-\left.\nabla[\mathcal{Q}(\mathbf{r}, t)+V(\mathbf{r}, t)]\right|_{\mathbf{r}=\mathbf{R}_{i}(t)}
$$

The quantum potential $\mathcal{Q}(\mathbf{r}, t)$ is computed from the test particle probability density histogram using the same structure grid and $n$-point difference formulas as used for helium DFT calculations.

The expectation values of $\mathbf{r}(t)$ and $\mathbf{v}(t)$ are often needed for visualization purposes

$$
\begin{gathered}
\langle\mathbf{r}(t)\rangle=\int d \mathbf{r} \mathbf{r} \chi^{2}(\mathbf{r}, t) \\
\langle\mathbf{v}(t)\rangle=\int d \mathbf{r} \mathbf{v}(\mathbf{r}, t) \chi^{2}(\mathbf{r}, t)=\frac{1}{m} \int d \mathbf{r} \mathbf{j}(\mathbf{r}, t)
\end{gathered}
$$

Furthermore, the energy of the impurity as a function of time is

$$
E(t)=\int d \mathbf{r}\left[\frac{1}{2} m v^{2}(\mathbf{r}, t)+\mathcal{Q}(\mathbf{r}, t)+V(\mathbf{r}, t)\right] \chi^{2}(\mathbf{r}, t)
$$

As an example application, Fig. 4 displays snapshots of the ${ }^{4} \mathrm{He}_{1000}$ droplet density on the $x-z$ plane following a sudden $1 \mathrm{~s}^{2}$ to $1 \mathrm{~s} 2 \mathrm{~s}$ excitation of a single helium atom (i.e. formation of $\mathrm{He}^{*}$ as indicated by the bright yellow spot in the figure) from bulk (15 $\AA$ from the center of the droplet) and surface $(18 \AA)$ locations. ${ }^{92}$ The He* atom ejected from the droplet is represented by $10^{6}$ test particle trajectories. Note that, due to the non-spherical liquid distribution at the droplet surface, the normally forbidden s-s transition becomes partially allowed.

\section{Simulation of absorption and emission spectra using the density fluctuation} method

Optical absorption and fluorescence spectroscopy of doped helium droplets establishes an important link between experiments and theory. Not only does it provide a test to validate the applied theoretical method, but it can also give a microscopic view into the associated dynamics. The latter aspect has, for example, been used to establish the details of impurity solvation in helium droplets (e.g. interior vs. surface solvation) ${ }^{95}$

Provided that the helium dynamics does not contribute to the spectrum significantly, the transition energies can be approximated with the eigenvalues of $U_{T}$ defined after Eq. 

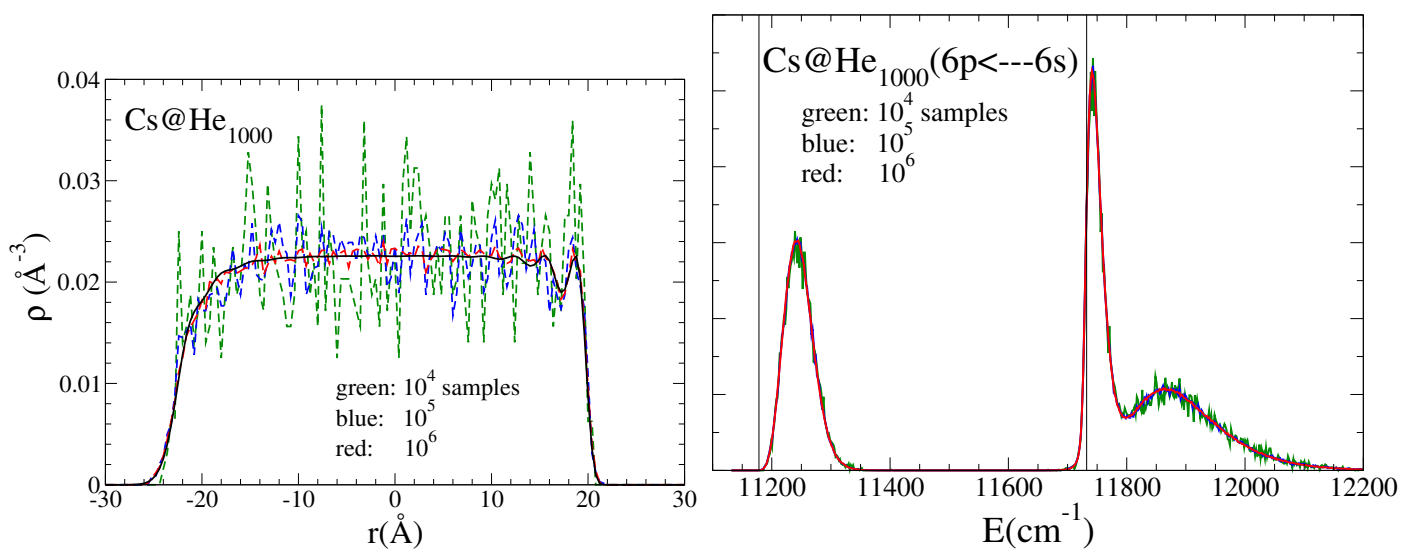

FIG. 5. (a) DFT density profile along the $z$ axis $[\rho(z)]$ of the $\mathrm{Cs}_{\mathrm{S}}{ }^{4} \mathrm{He}_{1000} \operatorname{droplet}$ (solid line) and the simulated profile corresponding to $n_{c}=10^{4}$ (green), $10^{5}$ (blue), and $10^{6}$ (red) simulations. (b) $6 p \leftarrow 6 s$ absorption spectrum of Cs obtained using the corresponding sampled density distribution. The Patil He-Cs potential ${ }^{93}$ has been used for the ground state and the Pascale He-Cs potentials for the excited states. ${ }^{94}$ The spectrum is given in arbitrary units; in the cases of $n_{c}=10^{4}$ and $10^{5}$, it has been multiplied by a factor of 100 and 10 respectively so that they can be compared to that of $n_{c}=10^{6}$.

41. Within this model, line broadening originates from fluctuations in the helium density and/or the zero-point density distribution of the impurity $\left|\phi\left(\mathbf{r}_{I}\right)\right|^{2}{ }^{97}$ An outline of the former case is given below ('DF sampling method').

Within the Born-Oppenheimer approximation, electronic and nuclear degrees of freedom are treated separately. The absorption and fluorescence line shapes can then be calculated by Fourier transforming the helium bath time-correlation function ${ }^{98}$ Within the semi-classical approximation, $\frac{82}{}$ the absorption line shape function $I(\omega)$ is

$$
I(\omega) \propto \sum_{m} \int \mathrm{d}^{3} \mathbf{r}\left|\phi^{\mathrm{gs}}(\mathbf{r})\right|^{2} \delta\left[\omega-\left(V_{m}^{\mathrm{ex}}(\mathbf{r}) / \hbar-\omega^{\mathrm{gs}}\right)\right]
$$

where 'ex' and 'gs' refer to the electronic excited and ground states, respectively. The DF sampling method constructs this expression stochastically by generating a large number of helium-impurity configurations $\left(n_{c} \approx 10^{6}\right)$. Each configuration consists of $N$ helium atoms positions and, if the impurity is light, its position as well. For helium, these coordinates 

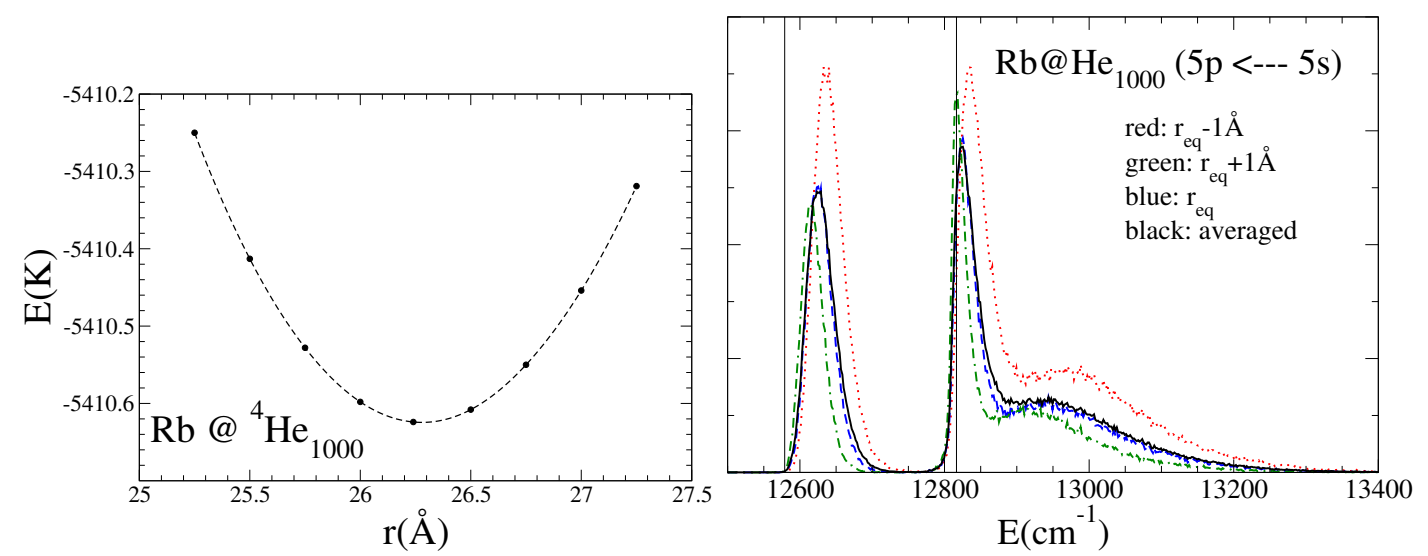

FIG. 6. (a) Energy of $\mathrm{Rb} @{ }^{4} \mathrm{He}_{1000}$ as a function of the distance of the $\mathrm{Rb}$ atom from the COM of the droplet; the equilibrium position of $\mathrm{Rb}$ is $r_{e q}=26.24 \AA$. (b) Absorption dipole spectrum (arbitrary units) for several locations of the Rb atom shown in (a). The number of configurations used to simulate the three spectra is $n_{c}=10^{5}$.

are randomly generated by importance sampling using the DFT helium one-particle density $\rho(\mathbf{r}) / N$ as the probability distribution, where short-range correlations from the hard-sphere term are also considered. The impurity positions are sampled using the zero-point distribution $\left|\phi^{\mathrm{gs}}\left(\mathbf{r}_{I}\right)\right|^{2}$. Such sampling is obviously not required for classical impurities.

Fig. 5 shows the one-particle density generated by importance sampling, compared to the calculated by DFT in the case of a (classical) Cs doped ${ }^{4} \mathrm{He}_{1000}$ droplet, using $n_{c}=10^{4}, 10^{5}$, and $10^{6}$ configurations. Examples considering the impurity quantum mechanically can be found in Refs. 97 and 99 .

To determine the contribution of each configuration $j$ to the overall absorption spectrum, the corresponding line position is computed from the difference between the excited and ground state energies. The latter is simply taken as the sum of pairwise ground state interactions, $V^{\text {gs }}\{j\}=\sum_{i} V_{X}\left(\left|\mathbf{r}_{i}^{\{j\}}-\mathbf{r}_{I}^{\{j\}}\right|\right)$, whereas the excited state energy is determined by the eigenvalues of $U_{T}=U+U_{S O}$, where $U_{T}$ was defined after Eq. (41) as the sum of the DIM [Eq. 40] ] and the SO hamiltonians. The absorption spectrum is finally constructed 
as an histogram of the line positions corresponding to each configuration

$$
I(\omega) \propto \sum_{m} \sum_{\{j\}}^{n_{c}} \delta\left[\omega-\left(V_{m}^{\mathrm{ex}}\{j\}-V^{\mathrm{gs}}\{j\}\right) / \hbar\right] .
$$

As an example, Fig. 5 shows the absorption spectrum for the Cs $6 \mathrm{p} \leftarrow 6$ s transition in a ${ }^{4} \mathrm{He}_{1000}$ droplet with $n_{c}=10^{4}, 10^{5}$, and $10^{6}$. Note that even $n_{c}=10^{5}$ appears to be sufficient to produce a good quality spectrum even though the sampled one-particle density has not fully converged yet. It should be stressed that other sources of broadening such as thermal motion,, 82 coherent helium bath dynamics,,$\sqrt[100]{ }$ or droplet size distribution can also contribute to line broadening and are not included in this model.

The influence of thermal motion on the absorption spectrum can be accounted for by considering a thermodynamic ensemble of doped droplets at the experimental temperature of $0.37 \mathrm{~K}$. This is illustrated in the following for $\mathrm{Rb}$ doped ${ }^{4} \mathrm{He}_{1000}$ droplets. By constraining the distance between $\mathrm{Rb}$ and the droplet $\mathrm{COM}$, the energy landscape seen by $\mathrm{Rb}$ can be computed as shown in Fig. 6. The energy corresponding to the experimental temperature of $0.37 \mathrm{~K}$ is obtained for distances of $\sim \pm 1 \AA$ away from equilibrium. Fig. 6 also shows the $\mathrm{Rb} 5 \mathrm{p} \leftarrow 5$ s absorption spectrum corresponding to selected displacements from equilibrium. Indexing them by $i$ and denoting the corresponding spectra by $I_{i}(\omega)$, the thermally averaged spectrum can be constructed as

$$
I(\omega)=\frac{1}{\mathcal{Z}} \sum_{i} I_{i}(\omega) e^{-\Delta E_{i} /\left(k_{B} T\right)}
$$

where $k_{B}$ is the Boltzmann constant, $\Delta E_{i}$ the energy difference from the equilibrium position, $\Delta E_{i}=E_{i}-E_{e q}$, and $\mathcal{Z}=\sum_{i} e^{-\Delta E_{i} /\left(k_{B} T\right)}$ is the partition function. At $0.37 \mathrm{~K}$ the thermally averaged absorption spectrum of $\mathrm{Rb}$ is very close to that obtained at the equilibrium position.

Finally, we note that fluorescence spectra can be calculated in a similar way by exchanging the roles of the ground and excited states. ${ }^{86}$ In this case the DF sampling employs the helium density around the impurity in its excited electronic state instead of the ground state.

\section{RECENT APPLICATIONS OF DFT FOR IMPURITY DOPED SUPERFLUID HELIUM}

This section gives an overview of selected results for impurity doped superfluid helium systems obtained with DFT over the past ten years. In addition to covering the wealth of 


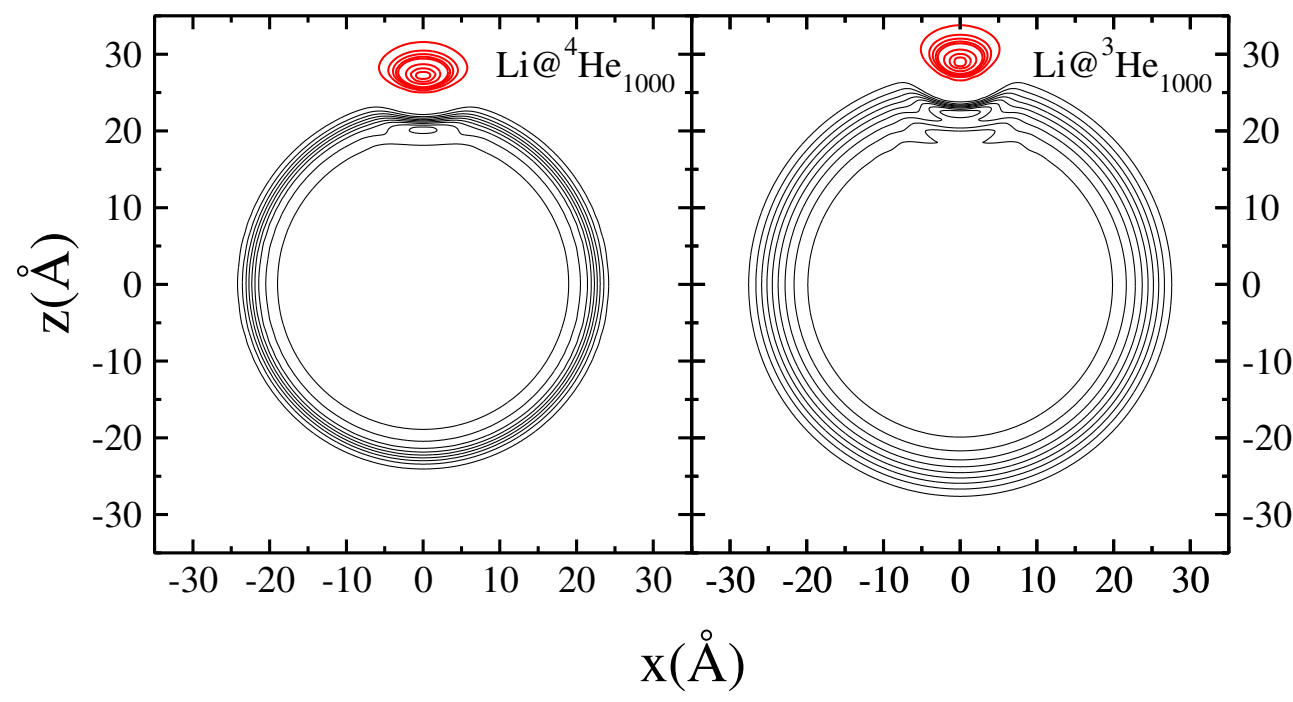

FIG. 7. Helium equidensity lines in a symmetry plane of a $\mathrm{Li}_{0} \mathrm{He}_{1000}$ droplet. There are nine lines with values ranging from $0.1 \rho_{0}$ to $0.9 \rho_{0}$ in $0.1 \rho_{0}$ steps with $\rho_{0}=0.0218 \AA^{-3}$ for ${ }^{4} \mathrm{He}$, and 0.0163 $\AA^{-3}$ for ${ }^{3} \mathrm{He}$. Also shown are equiprobability density lines for Li between $0.001 \times \max \left\{\left|\phi^{g s}\right|^{2}\right\}$ and $0.99 \times \max \left\{\left|\phi^{g s}\right|^{2}\right\}$. Left panel: Li@ ${ }^{4} \mathrm{He}_{1000}$. Right panel: Li@ ${ }^{3} \mathrm{He}_{1000} .99$

activity on helium droplets doped with alkali and alkaline earth metal atoms, which have been thoroughly studied from both experimental and theoretical points of view, special attention is paid on reviewing the real-time capture of simple atoms by helium droplets (with or without vortex lines) and the dynamics following excitation of impurities attached to helium droplets. Furthermore, other aspects that have also drawn much attention recently, such as soft-landing of doped helium droplets on solid surfaces and the appearance of vortex arrays in helium droplets, are included. Last but not least, impurity dynamics in liquid helium is also considered due to the recent activity in this area. The choice of these topics was motivated by the previous experimental work as well as their successful study by DFT or TDDFT. 


\section{A. Alkali metal doped helium droplets: solvation and absorption spectra}

Since quantum mechanics dominates the behavior of ${ }^{4} \mathrm{He}$ droplets, even the solvation of neutral atomic impurities depends on a subtle interplay between the impurity-helium interaction potential and the liquid energetics (e.g. surface tension). A simple procedure to predict whether an impurity solvates in superfluid helium (heliophilic) or resides on the surface of helium droplets (heliophobic) was introduced in Ref. 101. If the impurity is treated classically and interacts with helium through a simple Lennard-Jones potential ('spherical' impurity), the solvation behavior can be inferred from the value of a dimensionless parameter $\lambda$

$$
\lambda=2^{-1 / 6} \rho_{0} \epsilon r_{\min } / \gamma
$$

where $\gamma$ is the liquid surface tension, $\rho_{0}$ is the bulk density, and $\epsilon$ and $r_{\min }$ are the well depth and equilibrium distance of the Lennard-Jones potential, respectively. DFT calculations ${ }^{101}$ suggest that if $\lambda>1.9$ the impurity is heliophilic and solvates inside helium droplets, whereas if $\lambda<1.9$ the impurity is heliophobic and resides on the droplet surface instead. The validity of treating the impurity classically can be assessed by the de Boer parameter $\lambda_{d B}$

$$
\lambda_{d B}=\frac{h^{2}}{m \epsilon r_{m i n}^{2}}
$$

where $h$ is the Planck constant and $m$ is the impurity mass. For light impurities (e.g. H, Li, Na) $\lambda_{d B}>1$, whereas for heavier impurities that can be treated classically (e.g. Ar, $\mathrm{SF}_{6}$ ) the value of $\lambda_{d B}$ is typically less than 0.15 .

Based on Eq. (62), all alkali metal atoms have $\lambda$ values much lower than the threshold value $\lambda=1.9$, which indicates that they should reside on the droplet surface ${ }^{101}$ This prediction was confirmed by subsequent experimental work ${ }^{102}$ in which the observed impurity line positions were very close to their gas phase values. Surface location is a direct consequence of the very weak binding between alkali metal atoms and helium. The prediction based on Eq. (62) is less conclusive for alkaline earth metals. For example, $\lambda$ is very close to 1.9 for $\mathrm{Ca}, \mathrm{Sr}$, and $\mathrm{Ba}$, whereas a value of 2.6 is obtained for $\mathrm{Mg}$. All available experimental evidence indicates that the former species are located on the droplet surface. For $\mathrm{Mg}$ the value of $\lambda$ indicates that this species is heliophilic, which is confirmed by both DFT and QMC calculations. This is discussed further in Sec. VB.

Two major achievements of DFT applied to doped helium droplets are the determination 
of the resulting solvation structures and the associated optical spectra. In addition to the work reviewed earlier in Ref. 7, joint experimental-theoretical studies on alkali metal atoms from Li to $\mathrm{Cs}$ in both ${ }^{4} \mathrm{He}$ and ${ }^{3} \mathrm{He}$ droplets have been published since. Impurities were treated either classically (i.e. as an external field) ${ }^{103}$ or quantum mechanically $\underline{97 / 99}$ depending on their masses. Optical absorption spectra in these studies were computed from the Frank-Condon factors, $\frac{103}{1}$ the DF sampling method,,$\frac{97}{}$ or Fourier transformation of the time-correlation function. ${ }^{97 / 99}$ In addition, evaluation of time-dependent first-order polarization based on the superfluid helium response has been used for calculating the optical spectrum of intrinsic helium impurities,$\frac{100}{}$ which will be discussed in more detail in Sec. $\mathrm{V} \mathrm{H}$.

The above mentioned studies on alkali metal atoms have demonstrated good agreement with existing experimental results. The calculations were able to reproduce not only the general features of the absorption spectra for ${ }^{4} \mathrm{He}$ vs. ${ }^{3} \mathrm{He}$ droplets, but also the fine details observed for $\mathrm{Li}$ and $\mathrm{Na}$ coupled to either a bosonic or a fermionic helium surface. As an example, Fig. 7] shows the helium density and Li probability density on the symmetry plane of a Li@ $\mathrm{He}_{1000}$ droplet ('dimple' surface structure). The droplet surface region is contained between the inner and outer equidensity contour lines. Since both the surface tension and the equilibrium density of ${ }^{3} \mathrm{He}$ are smaller than for ${ }^{4} \mathrm{He}$, the surface width of ${ }^{3} \mathrm{He}$ droplets is larger. The resulting dimple solvation structure for other alkali metal-doped ${ }^{3} \mathrm{He}_{1000}$ and ${ }^{4} \mathrm{He}_{1000}$ droplets can be found in Fig. 3 of Ref. 103 .

The dimple solvation structure is deeper on a ${ }^{3} \mathrm{He}$ than on a ${ }^{4} \mathrm{He}$ surface. This is a direct consequence of the smaller surface tension of ${ }^{3} \mathrm{He}$, which also yields a wider surface region. A deeper dimple increases the interaction between the impurity and the droplet. For this reason, the absorption spectra exhibit larger blue shifts in ${ }^{3} \mathrm{He}$ vs. ${ }^{4} \mathrm{He}$ droplets. This trend has been observed experimentally and confirmed by DFT calculations, which are also able to reproduce the fine details in the spectra. For example, in addition to the different absorption line shifts observed for $\mathrm{Li} / \mathrm{Na}$ doped ${ }^{4} \mathrm{He}$ vs. ${ }^{3} \mathrm{He}$ droplets, the appearance of weak sidebands in ${ }^{4} \mathrm{He}$ is reproduced by DFT. ${ }^{97 / 99 \mid 103}$ This methodology has also been extended to molecular species such as $\mathrm{Li}_{2} \cdot 104$

Both DFT and QMC calculations for doped-helium systems require an accurate representation of the helium-impurity interaction as input. Since the excited electronic states are typically much higher in energy than the ground state, DFT calculation of solvation structures only requires the ground state interaction. For the spectroscopic applications discussed 
above, the corresponding excited state interaction with helium must also be known. Since a helium atom usually introduces only a small perturbation to the electronic structure of the impurity, the pairwise potential approximation is often very accurate. Pair potentials can be obtained with high accuracy from $a b$ initio electronic structure calculations such as full configuration interaction or coupled-cluster theory.

When the pair potential approximation is not sufficient, a perturbative configuration interaction (PCI) method can sometimes be employed!105 This method was used for excited states of alkali metal atoms where the electronic degrees of freedom couple significantly to the nearby helium atoms. PCI solves the electronic Schrödinger equation numerically in the valence orbital basis set for a free atom and includes an additional potential due to the valence electron-helium density interaction. This method can be applied to highly excited states of alkali metals where the conventional approach would fail. In a series of joint theoretical-experimental studies, it has been applied to model one- and two-photon spectroscopy of highly excited states of Rb, K, and Cs atoms in ${ }^{4} \mathrm{He}$ droplets, ${ }^{106}$ the spectroscopy of Rydberg states of $\mathrm{Na}$ atoms in ${ }^{4} \mathrm{He}$ droplets, ${ }^{107}$ and the photoionisation and imaging spectroscopy of $\mathrm{Rb}$ atoms attached to ${ }^{4} \mathrm{He}$ droplets. 108

As an example, Fig. 8 shows the PCI potential energy curves for $\mathrm{Na} @ \mathrm{He}_{2000}$. Based on these potential energy curves, the electronic excitation spectra of surface bound $\mathrm{Na}$ can be calculated and compared directly with experiments. This is demonstrated in Fig. 9 for one-photon excitation spectra of surface bound $\mathrm{Na}$, which were obtained by monitoring $\mathrm{Na}^{+}$, $\mathrm{NaHe}^{+}$, and $\mathrm{NaHe}_{2}^{+}$ion masses. ${ }^{[07}$ The level of agreement obtained is excellent when PCI potentials are employed. In contrast, simulations based on pairwise additive potentials (not shown) considerably overestimate the helium induced spectral shift. This difference can be attributed to helium-induced mixing of the electron configurations.107

Spectroscopy of alkali metal atoms located on the surface of helium droplets has provided a wealth of detailed information on these systems. ${ }^{9|13| 15}$ In addition to fluorescence excitation and emission spectra, angular distributions of the ejected atoms have been measured 80|107108 Considering the impurity-droplet system as a pseudo-diatomic molecule, these experiments can clearly distinguish between the $\Sigma$ and $\Pi$ states of the system. Alignment of the electronic angular momentum $\mathbf{j}$ for $\mathrm{Na}^{*}\left(3 \mathrm{p}^{2} \mathrm{P}_{3 / 2}\right)$ obtained by photoejection from ${ }^{4} \mathrm{He}_{200}$ droplets was modelled in Ref. 109. Together with the angular distribution parameter $\beta$, the coefficient for alignment of $\mathbf{j}$ was obtained from the simulation of the fragment state- 


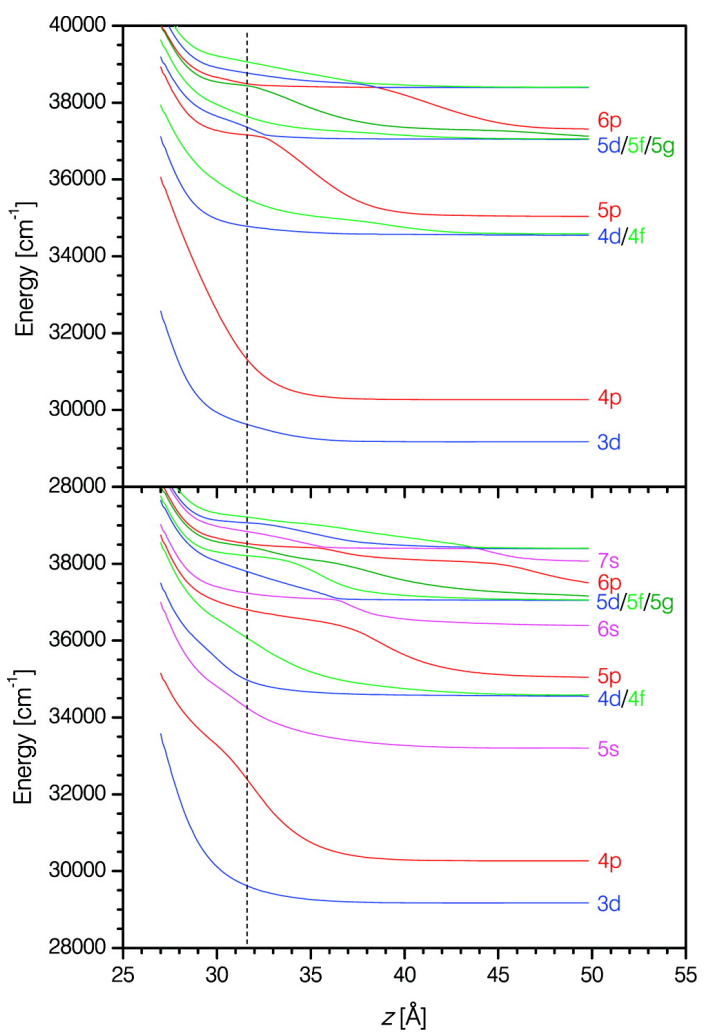

FIG. 8. Effective interaction potential curves obtained by the PCI method for a Na atom attached to a ${ }^{4} \mathrm{He}_{2000}$ droplet. Upper panel: $\Pi$ symmetry states. Lower panel: $\Sigma$ symmetry states. The dotted line gives the average distance between the sodium atom in the ground state and the center of the helium droplet. 107

resolved photoabsorption spectrum. The alignment coefficient exhibits clear oscillations as a function of the excitation energy. These oscillations were attributed to coherent population of the dissociative $\Sigma$ and $\Pi$ states within the Franck-Condon region. They could be observed experimentally through fluorescence polarization, provided that their dependence on the droplet size is not very strong as this could wash them out by averaging. They could also be visible in the photoelectron yields following ionisation of the atomic fragment. These predictions have not yet been confirmed by experiments.

The helium degrees of freedom are often involved in the relaxation of photoexcited impurities. As discussed before, optical excitation of $\mathrm{Na} 3 \mathrm{p} \leftarrow 3$ s transition populates the 


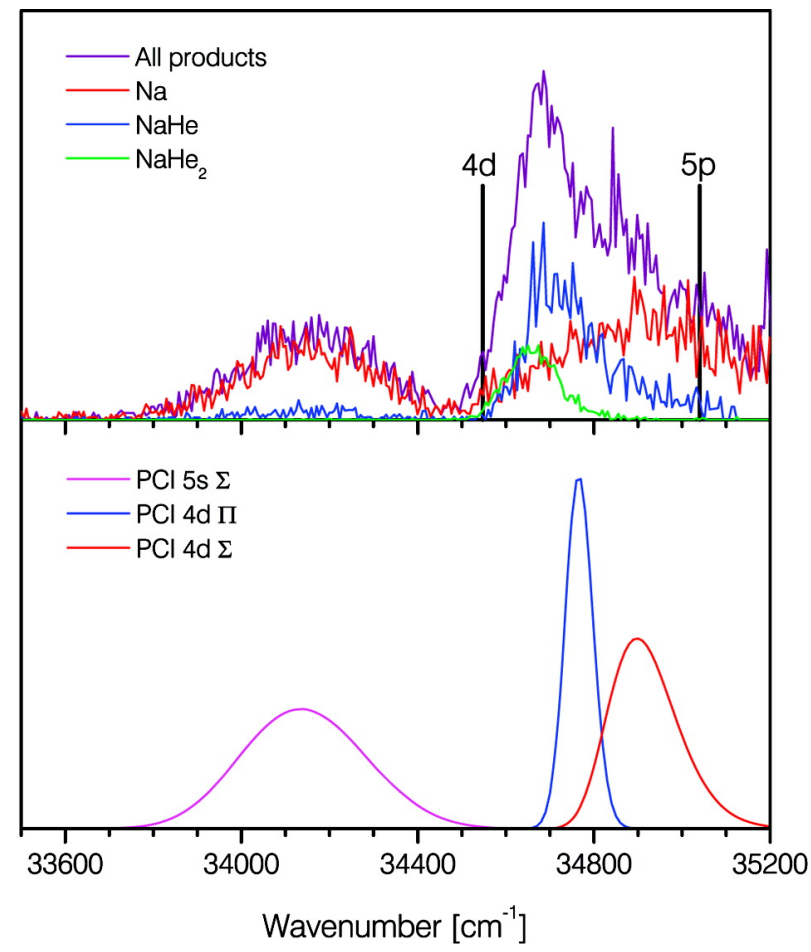

FIG. 9. Upper panel: Experimental excitation spectra of sodium attached to helium droplets recorded by monitoring the yield of $\mathrm{Na}^{+}, \mathrm{NaHe}^{+}$, and $\mathrm{NaHe}_{2}^{+}$ions (arbitrary units). The positions of the free atom transitions are indicated by vertical lines. Lower panel: Theoretical spectra obtained by DFT employing PCI potentials. 107

pseudo diatomic $\Sigma$ and $\Pi$ states on the droplet surface. It was shown that $\Pi$ state excitation produces both bare $\mathrm{Na}$ and $\mathrm{NaHe}_{n}$ exciplexes.110 Based on their measured velocity distributions, the bare $\mathrm{Na}$ atoms appear to be produced by an impulsive mechanism whereas exciplex production is thermally driven. The $\Sigma$ state is very repulsive and leads to impulsive desorption of bare $\mathrm{Na} \cdot \frac{110}{\sqrt{110}}$ Based on the spin-adiabatic approximation, these bare atoms should only be produced in the ${ }^{2} \mathrm{P}_{3 / 2}$ state. However, the experiment measured population in both the ${ }^{2} \mathrm{P}_{3 / 2}$ and the ${ }^{2} \mathrm{P}_{1 / 2}$ states. This has been attributed to a curve crossing taking place between the pseudo-diatomic states at long range. ${ }^{110}$ Similar curve crossings have also been reported for other alkali metal-rare gas systems.111 


\section{B. Alkaline earth metal doped helium droplets: solvation and absorption spectra}

Helium droplets doped with alkaline earth metals have been experimentally studied and modeled by DFT. Due to their larger binding towards helium as compared to heliophobic alkali metal atoms, the resulting solvation structure depends on the species considered and on the isotopic composition of the droplet. DFT calculations predict that alkaline earth atoms from $\mathrm{Mg}$ to $\mathrm{Ba}$ reside inside ${ }^{3} \mathrm{He}$ droplets; $\mathrm{Ca}$, $\mathrm{Sr}$, and $\mathrm{Ba}$ occupy dimple states on the ${ }^{4} \mathrm{He}$ droplet surface, and $\mathrm{Mg}$ is heliophilic. ${ }^{56}$ The DFT results are consistent with the available spectroscopic data for ${ }^{4} \mathrm{He}$ droplets, see Ref. 56 and references therein. No data is available for Be but it is presumably heliophilic.

Large helium droplets made up of a few thousand atoms may host vortex lines that are created during the gas condensation phase. Since trapping of impurities at vortex lines alters the surrounding liquid density distribution, it has been proposed that absorption spectroscopy of alkali metal atoms, excited state helium atoms, or electrons could be used to detect vorticity ${ }^{43] 1121113}$ Unfortunately, the spectral changes are predicted to be very small. For this reason, the Ca atom may be a better candidate because it is just barely localised on the droplet surface. $\frac{821144}{1 n}$ the presence of a vortex line, Ca atoms could be drawn into the vortex core and sink inside the droplet!115 Such a change in the solvation environment should produce a more pronounced effect in the absorption spectrum. Indeed, DFT calculations confirm this idea and the predicted changes in the absorption spectra are shown in Fig. 10$]^{822}$ However, the experimental absorption spectrum ${ }^{114}$ does not exhibit any structure that could be attributed to the presence of vortices to which $\mathrm{Ca}$ atoms are attached. It was concluded that the proportion of droplets with vortex lines in the beam is probably too small to produce a noticeable effect in the spectrum. Note that vortex arrays in large helium droplets have been observed experimentally 291161117 and modelled by DFT ${ }^{71}$ This will be discussed in more detail in Sec. VM,

Calculations of $\mathrm{Mg}$ atom-doped ${ }^{4} \mathrm{He}$ droplets have revealed an interesting solvation behavior as a function of the droplet size, indicating that $\mathrm{Mg}$ atoms are highly delocalised in the ${ }^{4} \mathrm{He}$ droplets. Indeed, DMC calculations for small droplets up to $N_{4}=50$ predict that $\mathrm{Mg}$ is not fully solvated below $N_{4} \sim 300^{[20121]}$ Recent PIMC calculations have found that $\mathrm{Mg}$ atoms are solvated in ${ }^{4} \mathrm{He}_{100}$ droplets. ${ }^{122]}$ thus confirming that the droplets must have at least several tens of helium atoms to fully solvate the $\mathrm{Mg}$ atom. DFT calculations for 


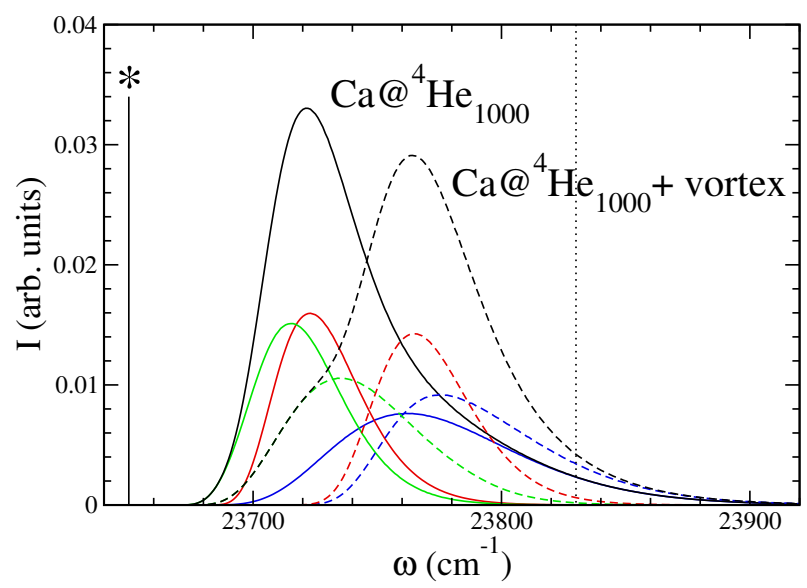

FIG. 10. Absorption spectrum of $\mathrm{Ca}$ in the ${ }^{4} \mathrm{He}_{1000}$ droplet with (dashed lines) and without (solid lines) a vortex line along the symmetry axis ${ }^{82}$ The absorption spectrum (black) is split into the $\Sigma$ (blue) and $\Pi$ components (green and red). The starred vertical line represents the gas-phase line position and the dotted vertical line represents the experimental value for bulk liquid ${ }^{4} \mathrm{He} .118$

small and large helium droplets ${ }^{56 \mid 119}$ are in agreement with the QMC findings. These results are also consistent with the analysis of Laser Induced Fluorescence (LIF) ${ }^{123}$ and Resonant Two-Photon-Ionisation (R2PI) experiments ${ }^{124}$ For very large droplets, $N_{4} \sim 10^{4}$, electronimpact ionisation measurements suggest that $\mathrm{Mg}$ atoms are located on the surface $\stackrel{125}{ }$ which is in clear disagreement with the previously mentioned LIF and R2PI experiments and with the calculations. The origin of this discrepancy has not been identified yet.

When ${ }^{4} \mathrm{He}$ droplets are doped with more than one impurity, their free motion and strong mutual attraction are expected to lead to efficient clustering inside the droplet. The formation and properties of metal clusters isolated in helium droplets has been reviewed in Ref. 11. If the long-range part of the impurity-impurity interaction becomes comparable to that of the impurity-helium interaction, a dilute loosely bound 'bubble foam' structure (also called 'quantum gel') may form Ref. 124. Such a foam consists of separated impurities trapped in their own solvation bubbles within the droplet. A similar scenario was put forward to explain experimental findings related to the successive capture of impurities in helium droplets ${ }^{126}$ and in the bulk liquid. 127

The first DFT calculation to model the formation of bubble foam in bulk superfluid 

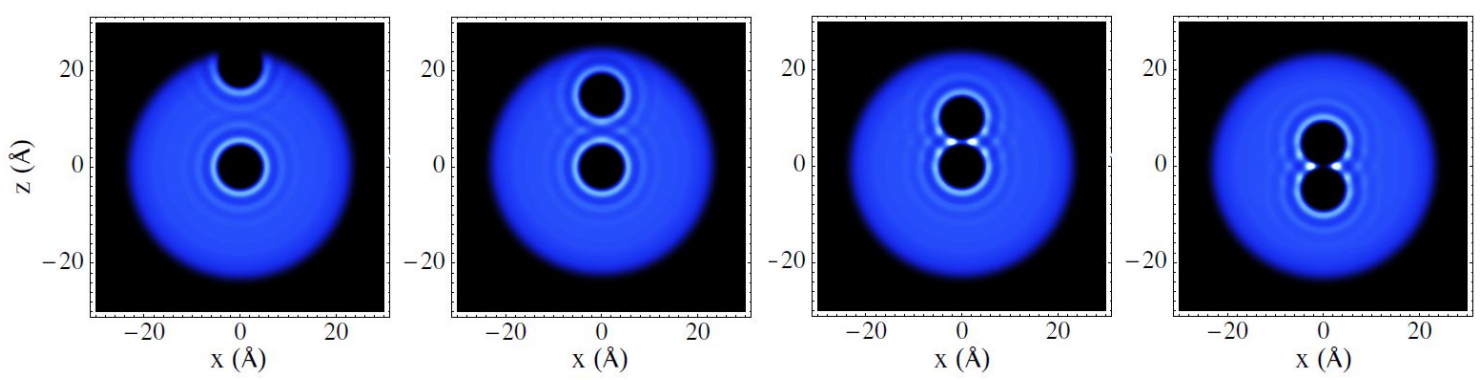

FIG. 11. From left to right, $(\mathrm{Mg}+\mathrm{Mg}) @{ }^{4} \mathrm{He}_{1000}$ metastable configurations for $\mathrm{Mg}-\mathrm{Mg}$ interatomic distances $18.5 \AA, 12.9 \AA, 9.3 \AA$, and $9.5 \AA$. The corresponding total energies are $-5567.8 \mathrm{~K}$, $-5573.9 \mathrm{~K},-5580.3 \mathrm{~K}$ and $-5581.4 \mathrm{~K}$, respectively. The bright regions correspond to high density helium. 119

helium was carried out for Ne atoms. ${ }^{128}$ The Ne-He interaction is strong enough to produce a localised solvent shell structure around Ne. The calculated interaction energy as a function of the distance between two Ne atoms, including the liquid contribution, exhibits local maxima when the solvent shells centered around each atom overlap. This creates a liquid induced energy barrier to recombination, which may localise the atoms far away from their gas phase equilibrium positions, provided the barrier is higher than the thermal energy. Similar calculations have been published for $\mathrm{Ag}-\mathrm{Ag}, \mathrm{Cu}-\mathrm{Cu}, \mathrm{Au}-\mathrm{Au}$, and F-F interactions in superfluid helium.129130 One of the goals in these studies was to address the timescale for metal-cluster formation in ${ }^{4} \mathrm{He}$ droplets by using a mixed DFT-classical molecular dynamics approach. 129

Motivated by the experimental work on multiply doped Mg droplets, 124 the above mentioned DFT approach was also used to study $\mathrm{Mg}-\mathrm{Mg}$ recombination in ${ }^{4} \mathrm{He}_{1000}$ droplets. 119 By carrying out the same calculation for ${ }^{3} \mathrm{He}$ droplets where the solvation shells are less pronounced, it was conclusively shown that the solvent shell structure around the impurity plays a key role in the foam formation. As an example, Fig. 11 shows several configurations for $(\mathrm{Mg}+\mathrm{Mg}) @{ }^{4} \mathrm{He}_{1000}$. Note that a ring of high density helium forms around the diatomic axis (see also Ref. 124 and 128). The extreme right configuration, where the Mg$\mathrm{Mg}$ distance is $9.5 \AA$, corresponds to the metastable foam configuration. At shorter $\mathrm{Mg}-\mathrm{Mg}$ 
distances the energy increases and prevents the recombination into the $\mathrm{Mg}_{2}$ dimer $\stackrel{[19]}{ }$ Based on experimental data, this metastable complex collapses into a tightly bound cluster in $c a$. 20 ps. ${ }^{124}$ The response of $\mathrm{Mg}$ atoms embedded in ${ }^{4} \mathrm{He}$ nanodroplets was later studied by femtosecond dual-pulse spectroscopy, which yielded results consistent with the hypothesis of isolated atoms arranged in a foam-like structure.131

The effect of the above bubble foam configurations on LIF and R2PI spectra in ${ }^{4} \mathrm{He}$ droplets was found to be in good agreement with the experimental data, as shown in Fig. 12 . ${ }^{119}$ The experiments show that doping helium nanodroplets with more than one $\mathrm{Mg}$ atom leads to a shift of the atomic absorption line from $279 \mathrm{~nm}$ to $282 \mathrm{~nm}$ due to the additional perturbation produced by the neighboring $\mathrm{Mg}$ solvation bubbles. $\frac{119}{10}$ It is worth mentioning that recent QMC calculations on the $\mathrm{Mg}$ pair in ${ }^{4} \mathrm{He}$ droplets did not yield any barrier for dimer formation $\stackrel{122}{122}$ no alternative interpretation for the R2PI experiments was presented.

The foam structures correspond to loosely bound clusters. Clusters may grow inside helium droplets with different structures, depending on the size of both the droplet and the cluster itself. The formation of Ag clusters up to a few thousand atoms in He droplets was studied via optical laser spectroscopy ${ }^{[133]}$ It was found that small Ag clusters $\left(N_{\mathrm{Ag}} \sim 100\right)$ exhibited a plasmon resonance at about $3.7 \mathrm{eV}$, similar to that previously obtained for dense spherical clusters. However, larger Ag clusters $\left(N_{\mathrm{Ag}}>1000\right)$ formed in ${ }^{4} \mathrm{He}_{N_{4}}$ droplets, $N_{4} \sim 10^{7}$, exhibited an unusually broad spectrum extending into the infrared spectral range. The dramatic change in the spectrum has been associated with a transition from singlecluster to multi-centre growth regime when the droplet size increases. The structure of the cluster aggregates formed inside He droplets remains unknown; it is conceivable that they are loosely packed and may even exhibit a fractal-like structure.

\section{Droplets doped with more than one species}

Helium droplets doped with two different impurity species with opposite solvation behavior have been investigated by DFT. Such studies have been inspired by experimental work showing that an otherwise heliophobic Ba atom could be solvated in helium droplets which already contain a heliophilic xenon cluster in their center.134

In a joint experimental and DFT work, ${ }^{135}$ droplets doped with HCN-M (M=Na, Ca, and Sr) have been studied. The calculations for these systems show a strong surface-bound state 


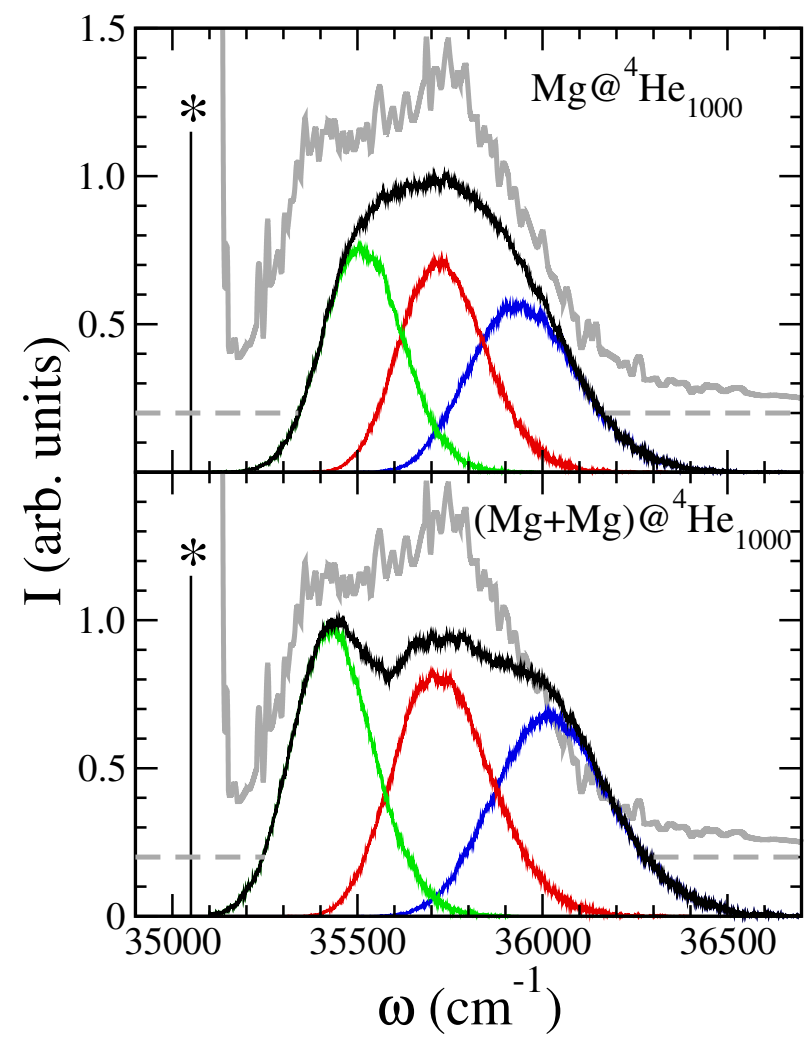

FIG. 12. Top panel: $3 \mathrm{~s} 3 \mathrm{p}{ }^{1} \mathrm{P}_{1} \leftarrow 3 \mathrm{~s}^{2}{ }^{1} \mathrm{~S}_{0}$ absorption spectrum of a single $\mathrm{Mg}$ atom in a ${ }^{4} \mathrm{He}_{1000}$ droplet. The starred vertical lines indicate the position of the corresponding gas-phase transition. The experimental curve is shown in grey ${ }^{\sqrt[123]{3}}$ Bottom panel: Same as top panel but the $\mathrm{Mg}$ atom resides in a distorted environment created by the presence of another nearby $\mathrm{Mg}$ atom.119[132

for $\mathrm{Na}$, a purely solvated state for $\mathrm{Ca}$, and both surface and solvated states separated by a barrier for Sr. The results for Ca and Sr were consistent with the appearance of the infrared spectrum for these complexes.

In another joint experimental and theoretical project, ${ }^{136 \mid 137}$ the influence of heliophilic argon doping on the solvation of heliophobic calcium atoms in helium droplets has been studied. The experiment considered the photodissociation of $\mathrm{Ca}_{2}$ to $\mathrm{Ca}\left(4 \mathrm{~s} 4 \mathrm{p}{ }^{1} \mathrm{P}\right)+\mathrm{Ca}\left(4 \mathrm{~s}^{2}\right.$ ${ }^{1} \mathrm{~S}$ ) in the presence of a varying number of Ar atoms in the droplet. The absorption and emission spectra of Ca- $\operatorname{Ar}_{M}(M=0-7)$ complexes were calculated by using the DF sampling method described in Sec. IVC with the Ca and Ar atoms treated classically. It was found 
that even a single Ar atom is enough to trigger Ca atom sinking into the He droplet, where they form a $\mathrm{Ca}_{2}$ dimer. Furthermore, by studying the emission spectrum as a function of the droplet size (Fig. 13), it was concluded that the emitting species was $\mathrm{Ca}^{*} \mathrm{Ar}_{M}$ attached to the droplet that has shrunk down to a size less than 200 helium atoms by either evaporation or detachment of helium atoms from the complex.

In another DFT study, ${ }^{138}$ a heliophilic Xe atom was placed in the bulk of a ${ }^{4} \mathrm{He}_{500}$ droplet with a heliophobic Rb atom located on the surface. The Rb-Xe van der Waals attraction was not sufficiently high to overcome the $23.4 \mathrm{~K}$ barrier induced by the presence of helium between the dopants and therefore Rb remained on the surface. Clearly, this is a dropletsize dependent effect. Furthermore, it was concluded that the order in which the dopants are introduced to the droplet plays an important role in the formation of such dimers, as they can only form on the droplet surface. In a recent study, ${ }^{139}$ evidence has emerged that sodium and cesium clusters, and even single Na atoms (but not Cs), can enter ${ }^{4} \mathrm{He}$ droplets (average size $N_{4} \sim 5 \times 10^{5}$ ) in the presence of a fully solvated $\mathrm{C}_{60}$ fullerene.

\section{Cluster-doped helium droplets}

Despite their practical and conceptual importance, $\frac{11}{11}$ theoretical studies simulating atomic clusters embedded in helium droplets are scarce. A major difficulty in these studies is to obtain reliable cluster-droplet interaction potentials. Besides the work on $\mathrm{Ca}-\mathrm{Ar}_{M}$ clusters mentioned before, $\frac{136 \mid 137}{{ }^{2}}$ the interaction of two Ne clusters in liquid ${ }^{4}$ He has been studied in Ref. 128. Furthermore, Path-Integral MC (PIMC) calculations of Mg and Na clusters in both helium droplets and the bulk liquid have been carried out ${ }^{140}$ These calculations show that $\mathrm{Mg}$ clusters are heliophilic whereas small $\mathrm{Na}_{M}$ clusters with $M=7,9$ remain on the surface. Recall that a single $\mathrm{Mg}$ atom is also heliophilic when $N_{4} \lesssim 30$.

Alkali atom clusters are especially interesting because the individual atoms reside on the droplet surface whereas larger clusters may become heliophilic and sink inside the droplet. The critical cluster size $N_{c}$ for switching from heliophobic to heliophilic behavior has been determined from the energy balance between the metal-helium van der Waals attraction, the short-range repulsion, and the liquid surface tension ${ }^{141}$ The following values have been

predicted for $N_{c}$ : Li,Na/ $/{ }^{4} \mathrm{He} \sim 20$; $\mathrm{Rb} /{ }^{4} \mathrm{He} \sim 100$; Li,Na/ $/{ }^{3} \mathrm{He} \sim 5$; and $\mathrm{Rb} /{ }^{3} \mathrm{He} \sim 20$ The values of $N_{c}$ in ${ }^{3} \mathrm{He}$ are smaller than in ${ }^{4} \mathrm{He}$ because of the lower value of the surface tension 


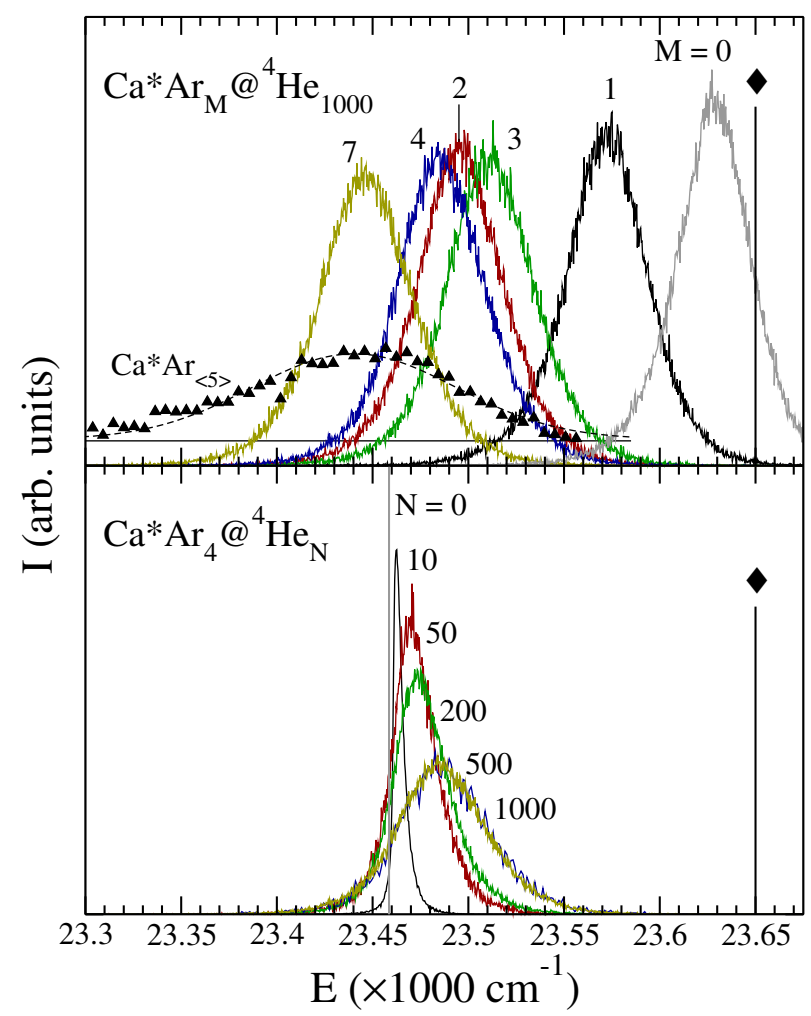

FIG. 13. Overview of $\mathrm{Ca}^{*}-\mathrm{Ar}_{M} \mathrm{He}_{N}{ }^{1} \mathrm{P} \rightarrow{ }^{1} \mathrm{~S}$ fluorescence emission spectra obtained by DFT. The upper panel shows the emission spectra for varying number of Ar atoms $M=1,2,3,4$, and 7 in a ${ }^{4} \mathrm{He}_{1000}$ droplet. The bottom panel shows the dependence of the $\mathrm{Ca}^{*}-\mathrm{Ar}_{4}$ spectrum on the number of helium atoms in the droplet $(N)$. The grey, vertical line labelled $N=0$ indicates the position of the isolated $\mathrm{Ca}^{*} \mathrm{Ar}_{4}$ vertical emission. The line labelled with a diamond is the atomic $\mathrm{Ca}^{*}$ emission line. The triangles in the upper plot are experimental data for $\langle M\rangle=5$ (hence a Poisson distribution of sizes with a weight of e.g. 0.03 for $M=1,0.08$ for $M=2,0.14$ for $M=3,0.18$ for $M=4$ and 5, 0.15 for $M=6,0.10$ for $M=7)$. The lower plot shows that, if the emitting species is inside a smaller droplet, the calculated spectra would shift by about $20 \mathrm{~cm}^{-1}$ to the red, giving a better agreement with experiment! $136 \mid 137$

and saturation density. The prediction for $\mathrm{Na}$ in ${ }^{4} \mathrm{He}$ droplets was later confirmed by the experiments, $\frac{142}{}$ a recent study on the submersion of $\mathrm{Na}$ clusters in ${ }^{4} \mathrm{He}$ and para- $\mathrm{H}_{2}$ clusters employing path-integral molecular dynamics has also found the submersion of $\mathrm{Na}_{N}$ clusters in ${ }^{4}$ He droplets around $N \sim 20,143$ 
Superfluidity of the helium surrounding $\mathrm{Mg}_{11}$ clusters in ${ }^{4} \mathrm{He}$ droplets consisting of up to a few hundred helium atoms has been studied by QMC ${ }^{144}$ Furthermore, the commensurateincommensurate transition of the ${ }^{4} \mathrm{He}$ atoms adsorbed on the surface of $\mathrm{C}_{20}$ and $\mathrm{C}_{60}$ was characterised by PIMC! $145[146$

In a more approximate way, the dissociation dynamics of neon clusters upon ionisation has been studied in a ${ }^{4} \mathrm{He}_{100}$ droplet using molecular dynamics corrected for delocalisation of the helium atoms and DIM based interaction potentials. 147148 The results showed two interesting processes, one in which the ionic core of the cluster, usually $\mathrm{Ne}_{2}^{+}$, is expelled from the rest of the droplet, and another showing a very efficient cooling effect by helium atom ejection rather than evaporation, with a wide kinetic energy distribution.

Sequential doping of helium droplets allows for the synthesis of core-shell clusters ('nanomatryoshkas'). Bimetallic clusters have been formed via sequential pickup of gold and silver atoms by helium droplets. $\frac{149}{149}$ The resulting structure persists upon 'soft-landing' of the clusters on a solid surface. Another nanomatryoshka, made of an Ag core coated by a shell of ethane molecules, has been studied.150 These systems are currently beyond the reach of a DFT-based description.

The DFT approach has also been used to simulate the solvation of single-walled carbon nanotubes consisting of up to 360 carbon atoms in a ${ }^{4} \mathrm{He}_{2000}$ droplet using an ab initio Henanotube interaction potential, $\frac{151}{2}$ see also Ref. 152. Depending on the nanotube diameter, the outer and inner walls are covered by one or more dense layers of helium. This structure, which was also found earlier on He-wetted graphite, $\stackrel{153}{1}$ forms as a consequence of the strong surface-He interaction and geometric effects. 154

\section{E. Doped mixed ${ }^{3} \mathrm{He}-{ }^{4} \mathrm{He}$ and ${ }^{3} \mathrm{He}$ droplets}

Experiments employing mixed helium droplets have been integral to the discovery of ${ }^{4} \mathrm{He}$ droplet superfluidity by rotational spectroscopy ${ }^{155}$ At low temperatures the two isotopes separate such that the inner part of the droplet consists of ${ }^{4} \mathrm{He}$ whereas ${ }^{3} \mathrm{He}$ resides on the outside.$^{7}$ Depending on the strength of the impurity-He interaction, the impurity may reside on the droplet surface, at the ${ }^{3} \mathrm{He}-{ }^{4} \mathrm{He}$ interface, or fully solvated inside the ${ }^{4} \mathrm{He}$ core. ${ }^{96 / 156}$

The structure and energetics of small mixed He droplets doped with $\mathrm{Mg}$ and Ca impurities has been studied by the quantum Diffusion Monte Carlo (DMC) method with the aim 


\section{$\mathrm{Ca} @{ }^{4} \mathrm{He}_{500}+{ }^{3} \mathrm{He}_{N_{3}}$}
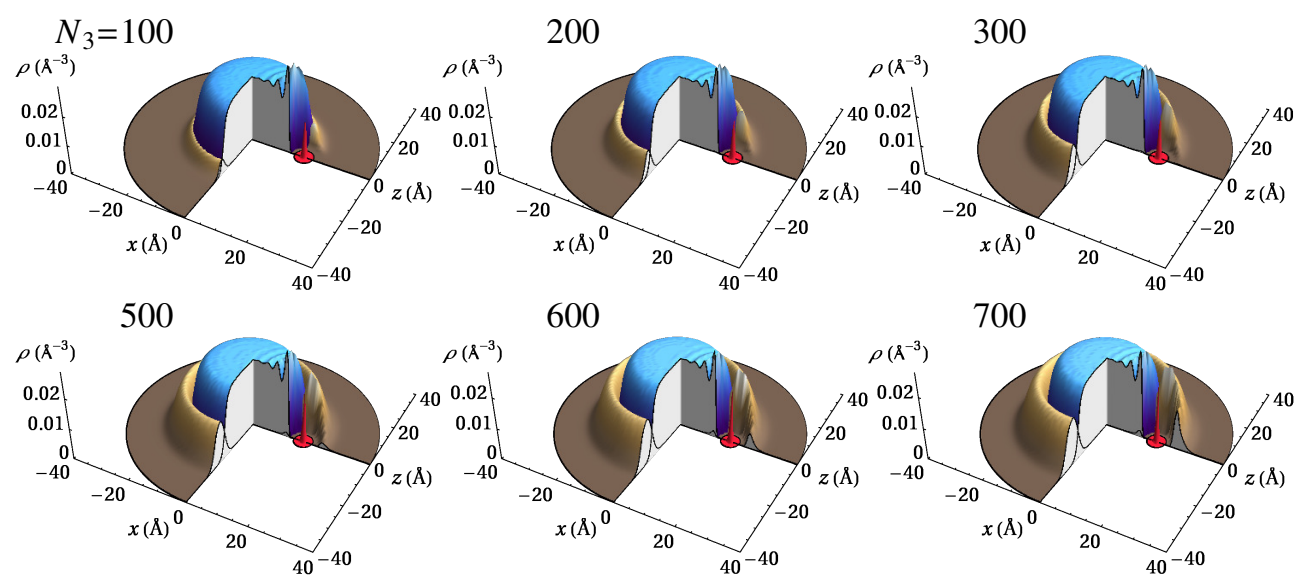

700

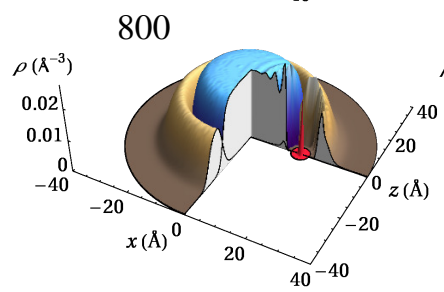

1000

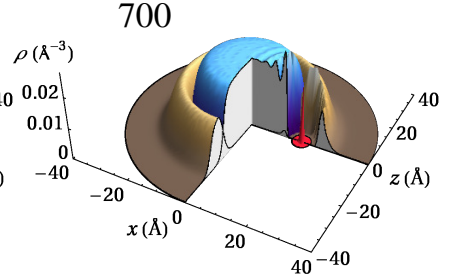

1500

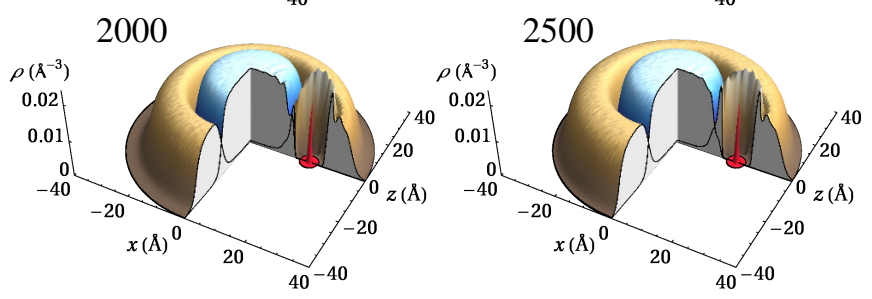

FIG. 14. A three-dimensional view of $\mathrm{Ca} @{ }^{4} \mathrm{He}_{500}+{ }^{3} \mathrm{He}_{N_{3}}$ helium droplets with $N_{3}$ varying from 100 to 2500 . The ${ }^{4} \mathrm{He}$ core and ${ }^{3} \mathrm{He}$ shell are pictured in blue and in brown, respectively. The probability density of the Ca atom is also displayed along the density cut (red spot; specified in arbitrary units).

of determining their solvation behavior in pure ${ }^{4} \mathrm{He}$ and ${ }^{3} \mathrm{He}$ droplets. ${ }^{121} 157$ Since $\mathrm{Ca}$ is heliophilic in ${ }^{3} \mathrm{He}$ droplets but heliophobic in ${ }^{4} \mathrm{He}$, it was expected to reach the ${ }^{4} \mathrm{He}$ core surface while remaining inside the ${ }^{3} \mathrm{He}$ shell. This is indeed confirmed by DFT calculations as illustrated in Fig. 14 for $\mathrm{Ca} @{ }^{4} \mathrm{He}_{500}+{ }^{3} \mathrm{He}_{N_{3}}$ droplets.

The interfacial location of $\mathrm{Ca}$ has also been verified by independent QMC calculations and absorption spectroscopy experiments. ${ }^{156 \mid 157}$ Figure 15 shows the calculated spectral shift 


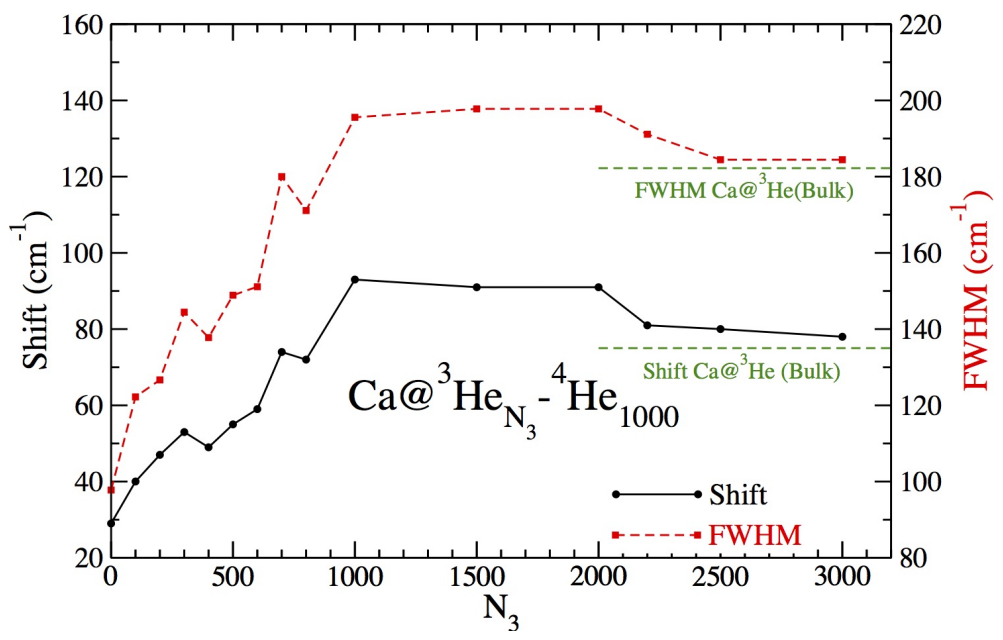

FIG. 15. Calculated shift and full width at half maximum (FWHM) of the Ca absorption spectrum around the $4 \mathrm{~s} 4 \mathrm{p} \leftarrow 4 \mathrm{~s}^{2}$ transition as a function of the number of ${ }^{3} \mathrm{He}$ atoms $N_{3}$ for $N_{4}=1000$

and full width at half maximum (FWHM) as a function of the number of ${ }^{3} \mathrm{He}$ atoms $N_{3}$ for $N_{4}=1000$. Direct comparison with experimental data is difficult because the ${ }^{3} \mathrm{He}^{-}{ }^{4} \mathrm{He}$ composition of the gas used may not directly carry over to the droplets $\frac{156}{15}$

Interatomic Coulombic decay (ICD $)^{158}$ has been proposed as a tool for studying the interface of isotopically mixed helium droplets doped with Ca atoms, $\frac{159}{2}$ since ICD is highly sensitive to the solvation environment. In a previous ICD study, isotopically pure ${ }^{3} \mathrm{He}$ and ${ }^{4} \mathrm{He}$ droplets doped with $\mathrm{Ne}$ and $\mathrm{Ca}$ were studied!160 The aim was to provide observables that would be sensitive to helium density around the impurity atom and compare them with DFT results. The first experimental study of ICD in ${ }^{4} \mathrm{He}$ nanodroplets, induced by photoexcitation of the $n=2$ excited state of ${ }^{4} \mathrm{He}^{+}$, has been carried out recently! ${ }^{161}$ It was found that the ${ }^{4} \mathrm{He}^{+}$kinetic energy distribution was strongly affected by the droplet environment, depending on whether ICD occurred inside the droplet or within the droplet surface region.

In DFT calculations of large mixed helium droplets, the kinetic energy of the ${ }^{3} \mathrm{He}$ component is treated using the Thomas-Fermi-Weizsäcker approximation, where the kinetic energy density is written as a sum of two terms, one proportional to $\rho_{3}^{5 / 3}(\mathbf{r})$ and the other proportional to $\left(\nabla \rho_{3}\right)^{2} / \rho_{3}$ ․ㅏㄹ ${ }^{162}$ However, this is only justified for large ${ }^{3}$ He droplets. For small 

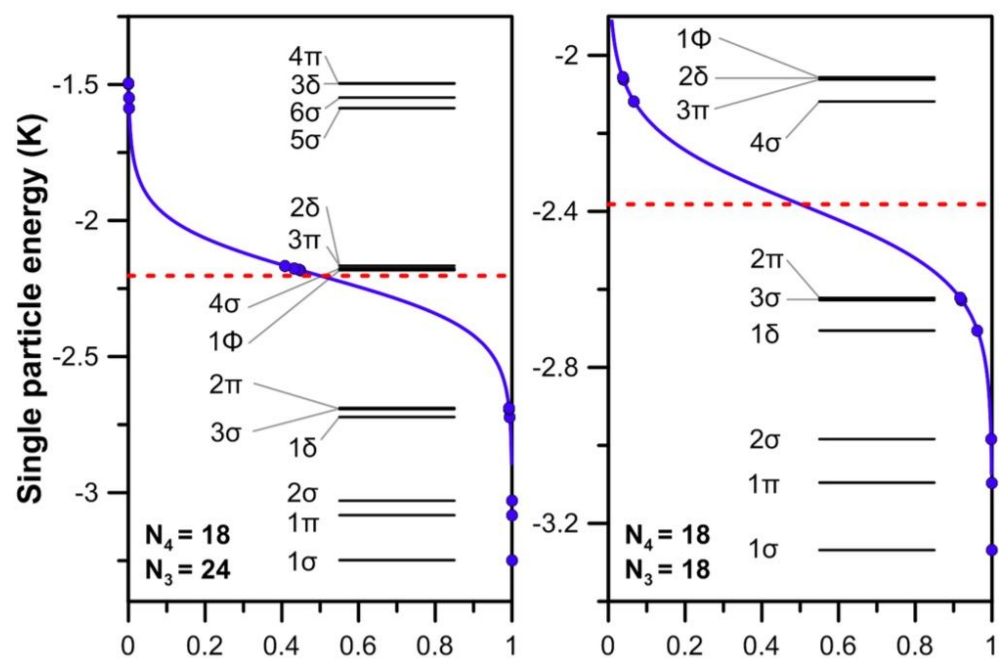

FIG. 16. Single-particle energies $\epsilon_{i}$ (vertical scale, K) of the ${ }^{3} \mathrm{He}$ component of an OCS@ ${ }^{3} \mathrm{He}_{N_{3}}+{ }^{4} \mathrm{He}_{18}$ droplet for two selected values of $N_{3} \cdot\left[\underline{55}\right.$ The dashed line represents the ${ }^{3} \mathrm{He}$ chemical potential $\mu_{3}$. The occupation numbers are represented by dots (horizontal scale) and the line connecting them represents the function $n_{i}=1 /\left\{1+\exp \left[\left(\epsilon_{i}-\mu_{3}\right) / k_{B} T\right]\right\}$.

droplets, the Kohn-Sham (KS) orbitals must be employed, which introduces an additional complication as the systems of interest are not spherical. DFT-KS studies have been published on small mixed helium droplets doped with $\mathrm{Ca} .163$ The single ${ }^{3} \mathrm{He}$ atom excitation spectrum in ${ }^{4} \mathrm{He}_{N_{4}}$ droplets with $N_{4}=8,20$, 40, and 50 has been obtained and compared with DMC results, $\frac{164}{1}$ and the effect on the ${ }^{3} \mathrm{He}$ excitation spectrum of doping the ${ }^{4} \mathrm{He}_{50}$ droplet with $\mathrm{Ca}$ was discussed.

Despite the conceptual relevance of addressing an OCS molecule embedded inside mixed helium droplets, DFT-KS calculations for the structure of small OCS@ ${ }^{3} \mathrm{He}_{N_{3}}+{ }^{4} \mathrm{He}_{N_{4}}$ systems, where the OCS molecule was treated as an external field, have only appeared recently One interesting aspect of this work is that ${ }^{4} \mathrm{He}$ has been described at $T=0$ whereas a finite temperature DFT-KS approach was used for ${ }^{3} \mathrm{He}$. This can be justified by considering that the elementary excitations of ${ }^{4} \mathrm{He}$ droplets are collective and their energies are of the order of several Kelvin, whereas the elementary particle-hole excitations of ${ }^{3} \mathrm{He}$ have energies comparable to the temperature of the experiment $\left(\sim 0.1 \mathrm{~K}\right.$ for OCS doped ${ }^{3}$ He droplets $) .165$ 


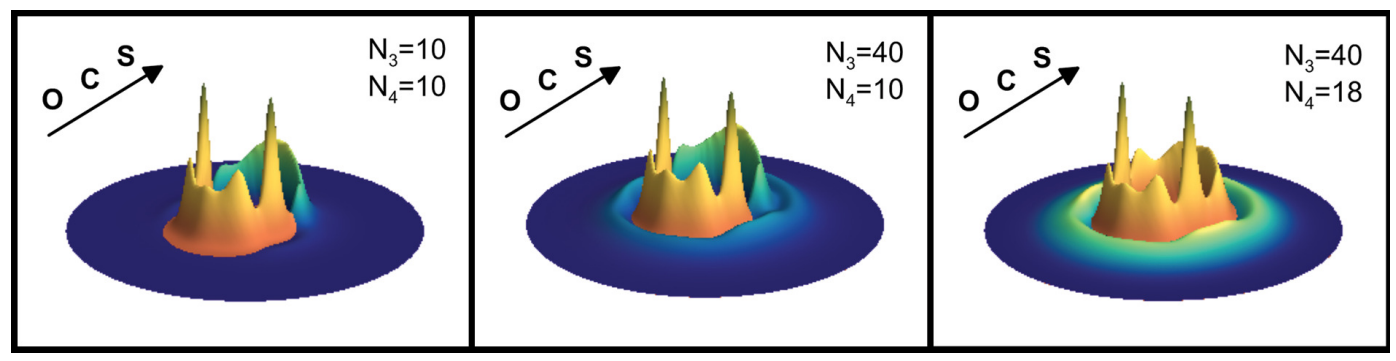

FIG. 17. Three-dimensional visualization of the atomic densities around OCS for mixed ${ }^{3} \mathrm{He}-{ }^{4} \mathrm{He}$ droplets $\stackrel{55}{5}$ The $N_{3}$ and $N_{4}$ values are indicated. Blue/green gradient represents the density of ${ }^{3} \mathrm{He}$ and orange/yellow gradient the density of ${ }^{4} \mathrm{He}$. The OCS molecule sits at the center and its orientation is indicated schematically.

Since mixed droplets cool down by evaporation from the ${ }^{3} \mathrm{He}$ free surface, a similar temperature to the previously mentioned particle-hole excitation energy is expected for mixed droplets. Such a small temperature has a negligible effect on the bosonic component of the droplet, but it may influence the fermionic component provided that the level spacing of the single-particle (s.p.) energy levels is of the order of $k_{B} T$. In this case, a large density of states with fractional occupation $n_{i}$ is expected around the Fermi level.

Given an ensemble $\left\{n_{i}\right\}$ that fulfills $N_{3}=\sum_{i} n_{i}$, the standard deviation of $N_{3}$ is given by

$$
\Delta N_{3}=\sqrt{\sum_{i} n_{i}\left(1-n_{i}\right)}
$$

This quantity exhibits pronounced local minima at ${ }^{3} \mathrm{He}$ shell closures ('magic numbers') and local maxima at $N_{3}$ values that correspond to half-filled shells. Notice that $\Delta N_{3}=0$ when all the occupation numbers are either 0 or 1 .

Figure 16 shows the s.p. structure of the ${ }^{3} \mathrm{He}$ component of an $\mathrm{OCS} @{ }^{3} \mathrm{He}_{N_{3}}+{ }^{4} \mathrm{He}_{18}$ droplet with $N_{3}=18$ and 24. Notice that $N_{3}=18$ corresponds to a closed-shell configuration whereas $N_{3}=24$ is a half-filled shell. Accordingly, the level scheme in Fig. 16 displays a fairly large energy gap around the Fermi level for $N_{3}=18$ and shows both large and small occupations. In contrast, the spectrum for $N_{3}=24$ is dense around the Fermi level with several s.p. states partially occupied. Since the energy gap is small in open shell droplets, their proper description must include thermal effects. Other examples are discussed in Ref. 


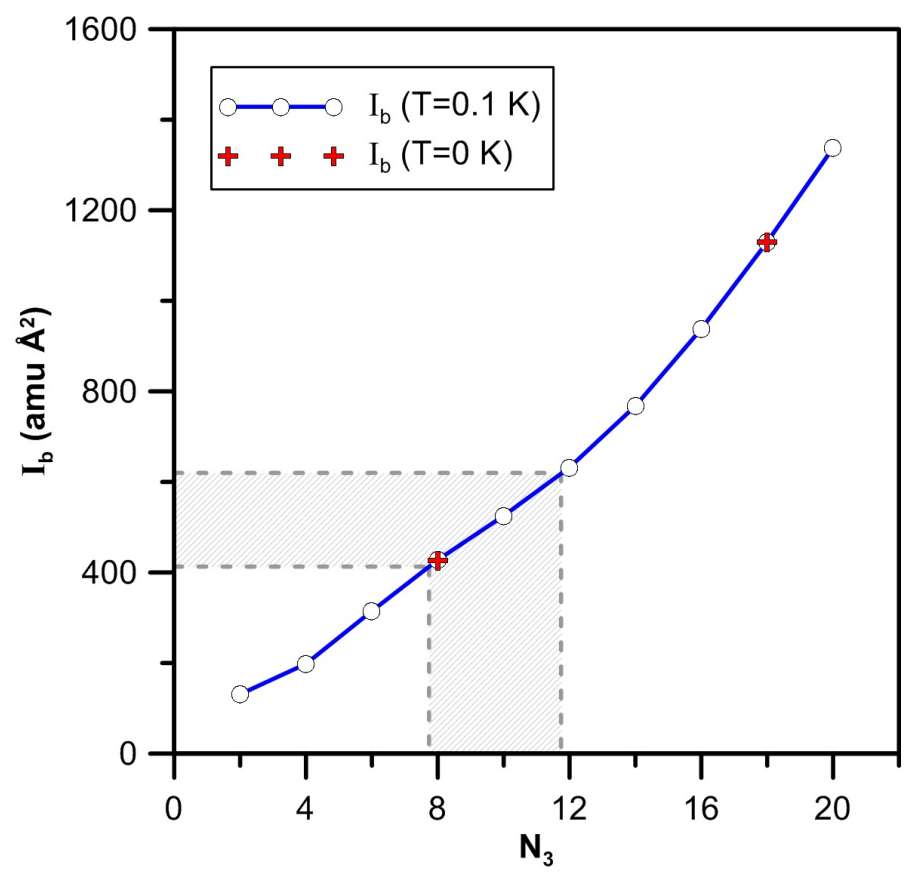

FIG. 18. Rigid moment of inertia of $\mathrm{OCS} @{ }^{3} \mathrm{He}_{N_{3}}$ (in amu $\AA^{2}$ ) perpendicular to the symmetry

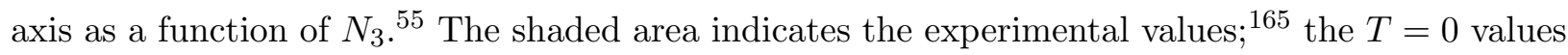
are from Ref. 166.

55. Helium density distributions around the OCS molecule for three selected configurations are shown in Fig. 17 .

Helium-3 droplets doped with OCS have been investigated within DFT-KS! 166 This work was motivated by the analysis of experimental infrared spectroscopy data for the OCS molecule embedded in ${ }^{3} \mathrm{He}_{N_{3}}$ droplets with $N_{3} \sim 1.2 \times 10^{4}$ ! ${ }^{155 \mid 165}$ Before this experiment, only the glyoxal molecule had been studied in ${ }^{3} \mathrm{He}$ droplets through the excitation of electronic and vibronic transitions. It was observed that the zero phonon lines (ZPL) were accompanied by additional broad bands on their red side due to particle-hole excitations of the droplet $\frac{167}{16}$ Furthermore, a small sharp peak superimposed on the additional band was assigned to vibrations of the snowball structure that surrounds the molecule. These particle-hole excitations accompanying the ZPLs were further analysed in Ref. 168.

While the Q-branch is missing in the infrared spectrum of OCS in both ${ }^{3} \mathrm{He}$ and ${ }^{4} \mathrm{He}$ droplets, the effective moment of inertia (MOI) in ${ }^{3} \mathrm{He}$ is approximately twice as large as 
compared to ${ }^{4} \mathrm{He}$. Note that this value is 5.5 times larger than observed in the gas phase. The increase in MOI was attributed to the presence of an evenly distributed shell of $11^{3} \mathrm{He}$ atoms around the molecule.

The structure and energetics of small OCS@ ${ }^{3} \mathrm{He}_{N_{3}}$ droplets was studied in Ref. 166 by using the functional described in Ref. 54. Similarly to the previously discussed calculations, the OCS molecule was incorporated into the model as an external potential. Since the calculation was carried out at $T=0$, it was restricted to small $N_{3}$ values that correspond to closed-shell OCS@ ${ }^{3} \mathrm{He}_{N_{3}}$ droplets, $N_{3}=8,18$, and 40 . The ${ }^{3} \mathrm{He}$ atoms are expected to fill the waist around OCS between the $\mathrm{O}$ and $\mathrm{C}$ atoms. The calculated number of atoms present in this ring was about 4 for ${ }^{3} \mathrm{He}$ droplets and 5 for ${ }^{4} \mathrm{He}$ droplets.

The distribution of He atoms around the OCS molecule affects its rotational properties. The MOI for OCS attached to ${ }^{3} \mathrm{He}_{N_{3}}$ droplets with $N_{3}=8,18$, and 40 was calculated by the rigid body expression and the results were compared with ${ }^{4} \mathrm{He}$ data. The calculated MOI of OCS@ ${ }^{3} \mathrm{He}_{N_{3}}$ is shown in Fig. $18{ }^{55}$ Comparison with $T=0$ results shows that the small non-zero temperature in the calculations does not influence the morphology of the fermionic droplet, but allows to carry out the calculations for any $N_{3}$ value and not only for the magic numbers.

\section{F. Electrons in liquid helium}

DFT has been succesfully used to study excess electrons and homogeneous cavitation in liquid ${ }^{4} \mathrm{He}$ and ${ }^{3} \mathrm{He}$. Analysis of phenomena such as the crossover from thermal to quantum cavitation in liquid ${ }^{4} \mathrm{He}$, heterogeneous cavitation by excess electrons, and the effect of vorticity on these processes have been presented in the literature 170,172

Any impurity embedded in liquid helium, including an electron, must loose its excess kinetic energy by ionisation of helium atoms and/or excitation of the liquid excitation modes. After the electron has lost most of its kinetic energy and moves at a velocity below the speed of sound in the liquid, it produces a cavity ('bubble') and becomes localised in it. In liquid ${ }^{4} \mathrm{He}$, this cavity - which is void of He atoms at $T=0$ - has a radius of $\sim 19 \AA$ and is the result of the competition between the zero-point energy of the confined electron, the surface energy of the bubble, and the work done against the liquid pressure for creating the cavity.

Figure 19 shows the helium density profile around the electron at $P=T=0$ along with 


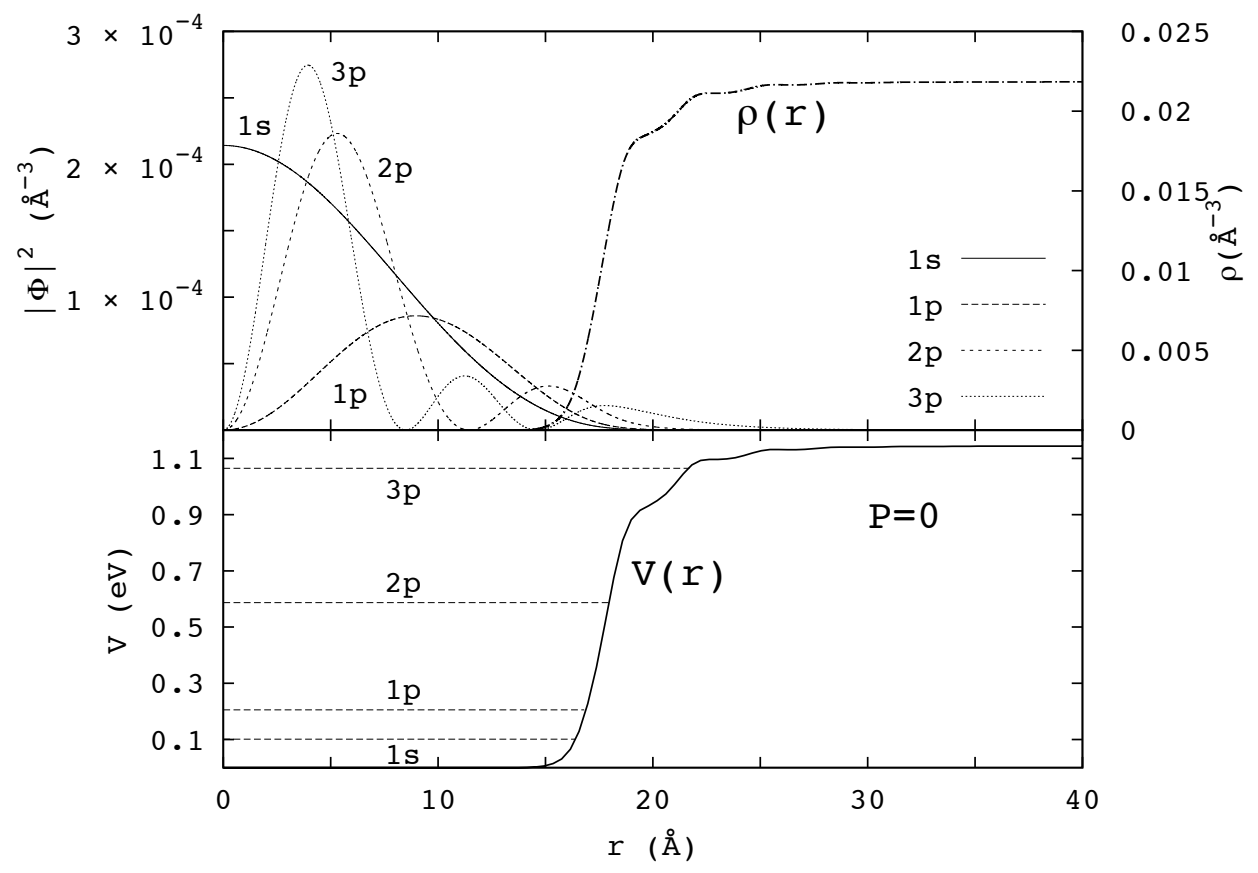

FIG. 19. Top panel: Electron bubble density profile (right scale) and electron probability density for the $1 \mathrm{~s}, 1 \mathrm{p}, 2 \mathrm{p}$ and $3 \mathrm{p}$ states (left scale) at $P=T=0$ in superfluid ${ }^{4} \mathrm{He}$. Bottom panel: The corresponding confining potential well and single-electron energies. ${ }^{169}$

the electron probability densities for $1 \mathrm{~s}, 1 \mathrm{p}, 2 \mathrm{p}$, and $3 \mathrm{p}$ states, and the confining potential. The 3p state is barely bound under SVP as its energy is just below the free electron limit, and it becomes delocalised above 1.7 bar $\frac{169}{15}$ The energy differences between the np and 1s electron levels correspond to the peak maxima in the electron absorption spectrum.

The properties of electron bubbles (e-bubble) in liquid helium have been reviewed in Ref. $172-176$ with the more recent articles concentrating specifically on the identification of the experimentally observed unusual negative species ('exotic ions'). Furthermore, multielectron bubbles in liquid helium have also been studied and their properties reviewed in Ref. 177. It was shown that highly charged multi-electron bubbles are unstable against fission at positive pressures. $\frac{178}{1}$ The production of long-lived multi-electron bubbles at negative 
pressures has been reported recently ${ }^{[179}$ A major difficulty in these studies is that the radius of the bubble must be on the micron-scale so that the electron-electron Coulomb repulsion can be reduced. So far, only two-electron bubbles have been modelled with DFT, 180 and the conclusion was that they are unstable and break into two separate electron bubbles. No real-time simulation of this process has been carried out yet.

While there is no direct way to measure the properties of electron bubbles, absorption measurements can provide relative energetics for the electron level structure. The DFT calculations performed in Refs. 181 and 182 are in excellent agreement with the experimental absorption data of Grimes and Adams $\frac{183}{183}$ Furthermore, DFT calculations are able to reproduce the experimentally observed negative pressure required to explode electron bubbles in the liquid. $\frac{184 \mid 185}{18}$ These comparisons confirm that the combined electron and DFT model can capture the essential physics of the e-bubble state.

The structure of e-bubbles hosting an excited electron has been a subject of interest since the experimental work in Refs. 172 and 187 was interpreted to involve such states. Furthermore, these bubble structures determine the emission spectrum of the electron bubble. The relevant DFT work on excited e-bubbles was carried out in Refs. 49, 180, and 186. The most interesting excited states are the $1 \mathrm{p}$ and $2 \mathrm{p}$ states, which can be accessed by experiments. The evolution of the bubble around these states has been computed by DFT.186 In this calculation, the liquid degrees of freedom evolve in real time while a time-independent Schrödinger equation is solved for the $n$ p electron at each time step. This adiabatic approximation can be justified by the large helium-electron mass ratio, $m_{4} / m_{e} \sim 7300$. Note that this approximation would fail if level crossings are encountered during the time evolution.

The adiabatic evolution of a $1 p$ e-bubble at $P=0$ and 5 bar is shown in Fig. 20 along with the quasi-static configurations obtained by fully relaxing the liquid. At $P=0$ the adiabatic evolution leads to a quasi-equilibrium configuration whereas at $P=5$ bar the e-bubble splits at the waist. The threshold pressure for the bubble splitting process is $\sim 1$ bar. This in agreement with the experiments, which indicate that the relaxed 1p bubble is only stable when pressure is smaller than $c a .1$ bar $\underline{188}$

The evolution of a $2 \mathrm{p}$ state e-bubble at $P=0$ has been studied within the adiabatic approximation. ${ }^{[186}$ The calculations revealed that after $7 \mathrm{ps}$, the $m=0$ levels of the $2 \mathrm{p}$ and If states become very close and the adiabatic approximation fails. This indicates that quasistatic electron bubble configurations above 1p cannot be reached. A detailed discussion on 

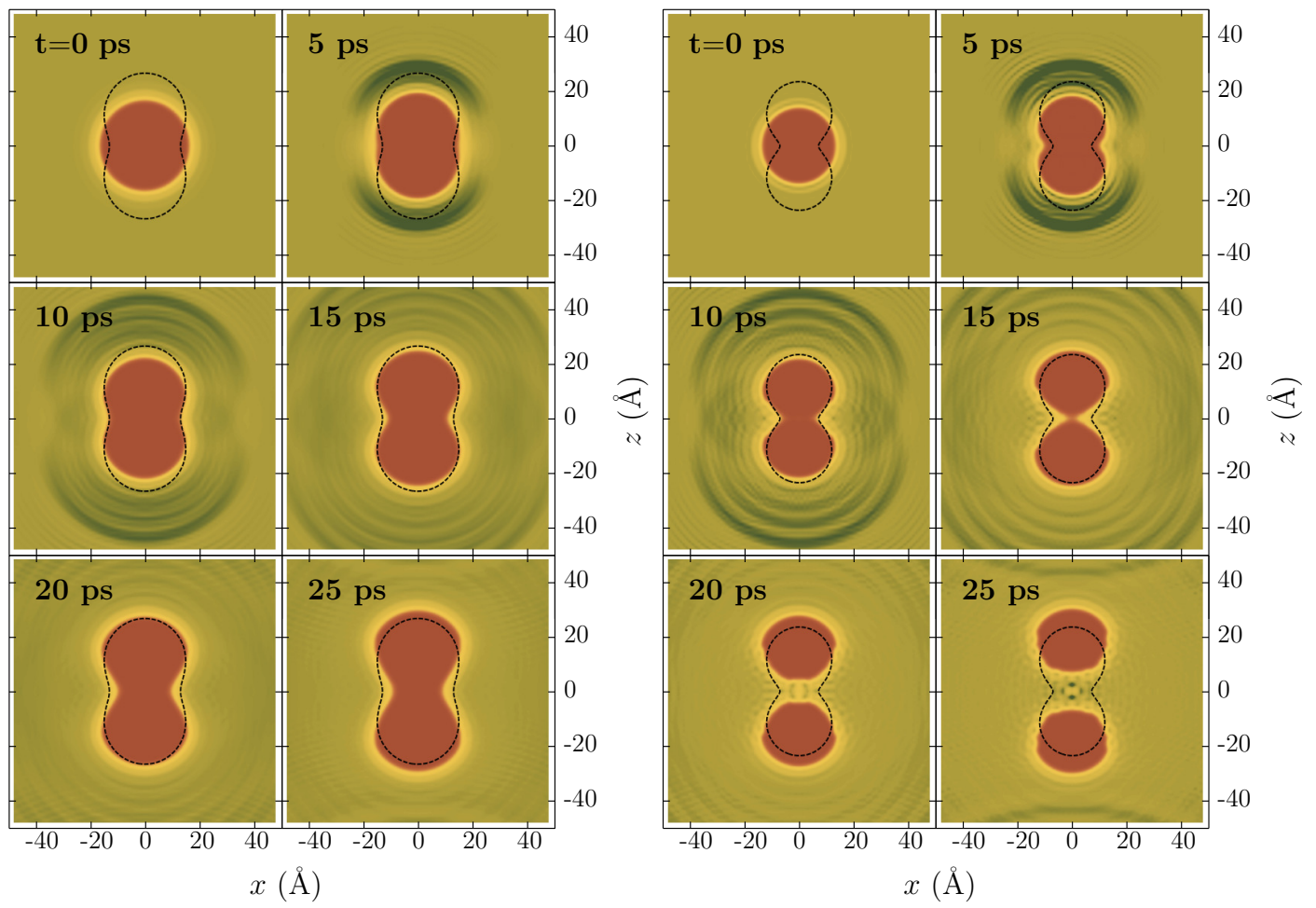

FIG. 20. (a) Adiabatic evolution of the $1 \mathrm{p}$ e-bubble at $P=0$. The panels display the helium configurations at the indicated times. The dashed line represents the dividing surface, which corresponds to half of the helium equilibrium density for the quasi-equilibrium configuration at $P=0$. (b) Same as (a) but for $P=5$ bar. 186

the validity of the adiabatic approximation for the electron bubble can be found in Ref. 186 and references therein. Other excited e-bubbles have also been studied by the quasi-static approximation. $172[180$

Real-time propagation of e-bubbles using finite-range functionals such as OT-DFT is computationally unfeasible due their complexity. The use of a zero-range functional as e.g. that of Refs. 25 and 26 (ST functional) simplifies the calculation of the mean field potential and reduces the computational demand of the calculation. ${ }^{46}$ A major shortcoming of zero-range functionals is that they cannot reproduce the maxon-roton portion of the liquid dispersion relation, but only the phonon part up to $q \sim 0.6 \AA^{-1} \underline{46}$ Despite this limitation, the evolution of $1 p$ electron bubbles at various pressures with both the zero and finite- 

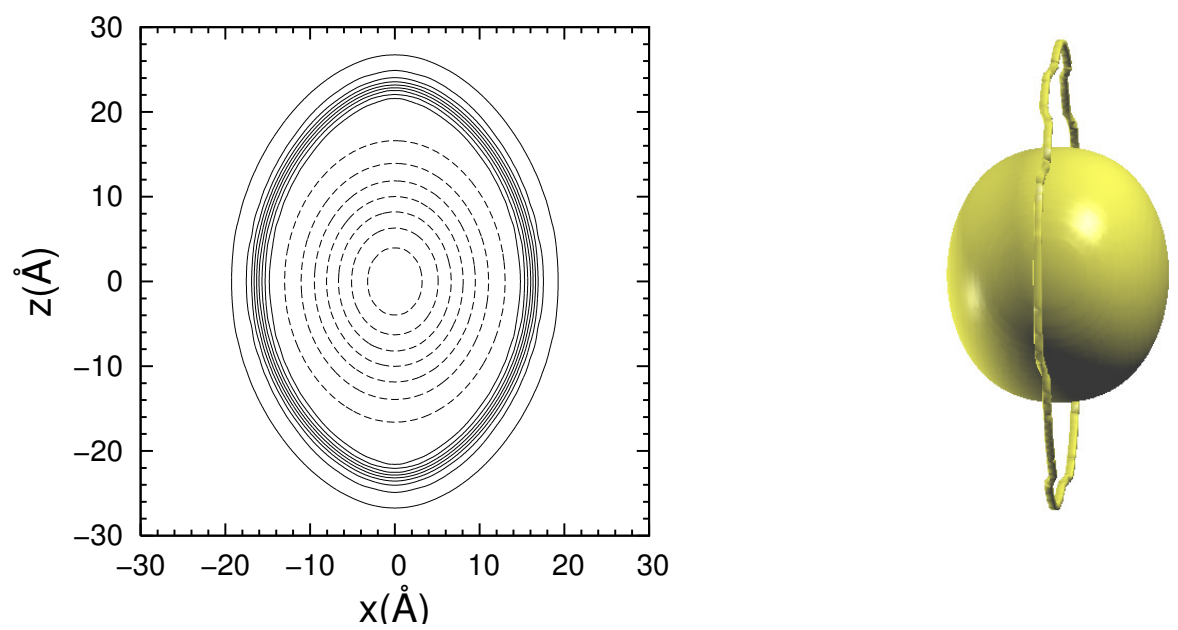

FIG. 21. (a) Contour plot showing the stationary state of the e-bubble at $P=0$ corresponding to $v=50.5 \mathrm{~m} / \mathrm{s}$, which is just below the critical value of $v_{c}=50.7 \mathrm{~m} / \mathrm{s}$. The equidensity lines for the ${ }^{4} \mathrm{He}$ density (solid lines) are plotted for values between $0.1 \rho_{0}$ and $0.9 \rho_{0}$ in steps of $0.1 \rho_{0}$ with $\rho_{0}=0.0218 \AA^{-3}$. The equidensity lines for the electron probability density (dashed lines) are plotted using nine lines between zero and its maximum value. (b) Surface isodensity plot showing a quantised vortex ring emitted at $P=0$ just above $v_{c} \cdot 41$

range functionals appear fairly similar. This suggests that zero-range functionals can also be applied to study $2 \mathrm{p}$ electron bubbles.

Real-time dynamics calculations employing the ST functional have confirmed the two key findings obtained using the adiabatic OT-DFT approximation: 1) the splitting of the 1p e-bubble above $P \gtrsim 1$ bar and 2) the failure of the adiabatic approximation for states higher than $1 \mathrm{p}$. The latter finding confirms that quasi-static configurations corresponding to $2 \mathrm{p}$, $2 \mathrm{~d}, \ldots$ do not exist.

Motion of electrons in liquid ${ }^{4} \mathrm{He}$ has been studied by Maris et al. using zero-range 
functionals $\stackrel{4748}{ }$ They showed that a 1 s electron bubble moving at a sufficiently high velocity begins to expand, deforms from the spherical symmetry, and nucleates vortex rings. Energy dissipation did not only arise from the creation of vorticity but also from the excitation of bubble surface modes. These results were confirmed by imaginary-time OT-DFT calculations in the co-moving frame, see Eq. (67).41189 Based on these calculations, the critical velocity for vortex ring nucleation at $P=0$ is $v_{c}=50.7 \mathrm{~m} / \mathrm{s}$. This value is in agreement with the critical electron drift velocity measured at low pressures. ${ }^{190}$

The complete mechanism of ring vortex nucleation is not yet fully understood. Based on DFT calculations, the e-bubble becomes compressed along the axis of propagation and elongated in the perpendicular direction upon increasing its velocity. Once the bubble exceeds the critical velocity $v_{c}$, a quantised vortex ring emerges from the equator of the bubble where the local liquid velocity is higher. Fig. 21 shows electron bubbles moving at velocities of $50.5 \mathrm{~m} / \mathrm{s}\left(<v_{c}\right)$ and slightly above $v_{c}$ (vortex ring emission). Note the significant distortion of the electron bubble geometry that appears below $v_{c}$ (cf. Fig. 19). The interaction of electrons with vortex lines is discussed in Sec. VL1.

\section{G. Cations in liquid helium and droplets}

Development of new techniques for doping helium droplets with charged impurities allow the controlled study of positive ions in superfluid helium. ${ }^{192}$ While neutral species could be excited in helium droplets by a resonant laser and detected essentially against a zero background, similar experiments have had limited success for ions. It is only recently that experimentalists have found a way to study the dynamics of photoexcited ions in helium droplets. This technique relies on the ejection of photoexcited ions from the droplets, which increases the yield of unsolvated ions at resonant wavelengths. $\frac{193}{19}$

Strongly attractive ions tend to form a solid-like helium layer around them (snowball). Alkali metal ions are believed to belong to this category ${ }^{191 \mid 195}$ In contrast, singly charged alkaline earth cations are expected to produce a cavity due to the outer electron-helium repulsion; thus, they are surrounded by a compressed but less inhomogeneous liquid! 32 |191|196

Alkali metal, alkaline earth metal, and rare gas cations have been recently studied by both experiments and theory ${ }^{197 / 198}$ A DMC investigation of $\mathrm{Pb}^{+}$in small He droplets, motivated by the experimental findings of Refs. 11 and 199, is reported in Ref. 200. A similar study 

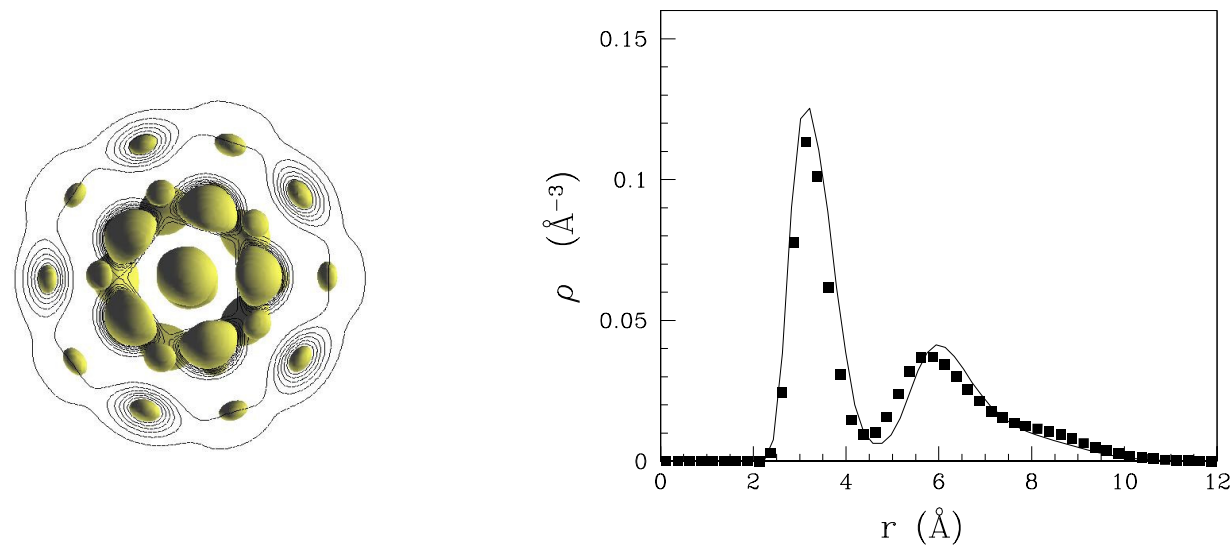

FIG. 22. (a) Helium density distribution around a $\mathrm{Be}^{+}$ion in a ${ }^{4} \mathrm{He}_{70}$ droplet shown by constant density surfaces $\rho=0.04 \AA^{-3} \underline{62}$ The lines show equidensity contours along a plane passing through the center of the droplet. (b) Average radial helium density of the $\mathrm{Be}^{+} \mathrm{Q}^{4} \mathrm{He}_{70}$ droplet. Solid line: DFT result; squares: PIMC result! 191

was carried out on $\mathrm{Na}^{+} .201$ These studies have highlighted the importance of the manybody interactions that arise from charge-induced dipole interaction between the ion and the surrounding helium atoms. Note that this interaction cannot be accounted for by including just the $-\alpha e^{2} /\left(2 r^{4}\right)$ polarization term in the cation-helium pair interaction.

DFT calculations have been used to study the solvation structure of $\mathrm{Ba}^{+}$cation in liquid helium and the stability of the so-called 'scolium'. Scolium, which was named after Giacinto Scoles, ${ }^{9}$ consists of an electron orbiting around a small helium droplet that hosts a positively charged ion. The electron cannot penetrate inside the droplet because of the large solvation energy barrier $(c a .1 \mathrm{eV})$. It was suggested ${ }^{62}$ that for small droplets, the pressure exerted by the orbiting electron further increases the local helium density around the ion due to electrostriction, which consequently turns the whole droplet into a solid. It was also shown that the lowest scolium state is unstable, the cation being pulled off from the droplet center towards the surface where it undergoes fast charge neutralization. The neutralization time was estimated to be on the order of a few picoseconds for a $50 \AA$ radius droplet. As an example of cation solvation in a helium droplet, Fig. 22 shows the helium density for a $\mathrm{Be}^{+}$ doped ${ }^{4} \mathrm{He}_{70}$ droplet where the ion is located at the center ${ }^{62}$ Comparison with the QMC data 


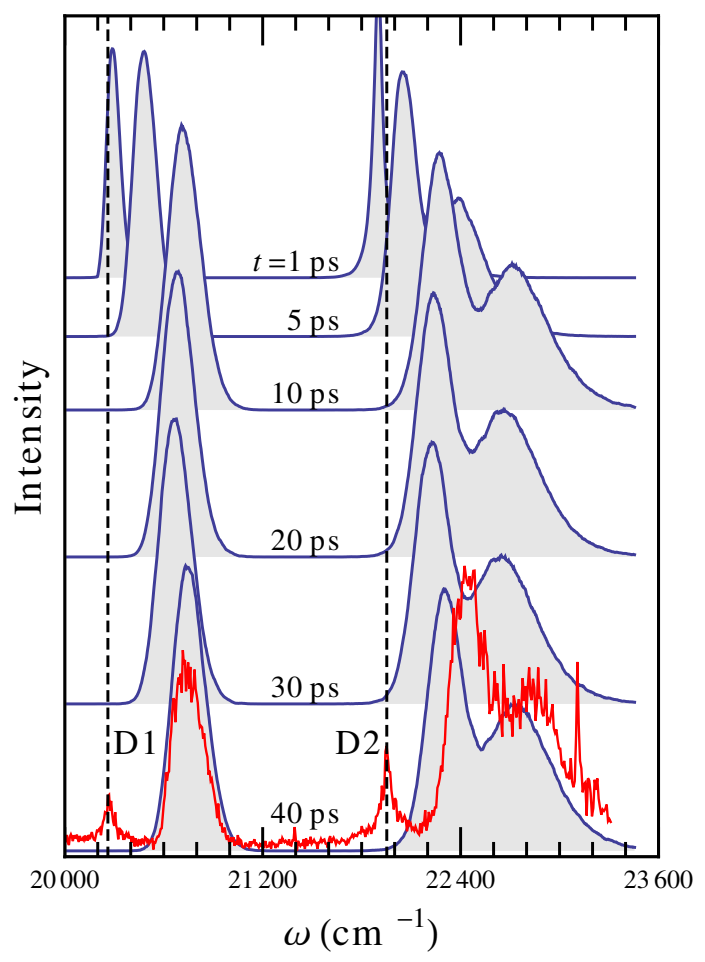

FIG. 23. TDDFT time-resolved absorption spectrum of $\mathrm{Ba}^{+}$in a ${ }^{4} \mathrm{He}_{1000}$ droplet. ${ }^{77}$ The experimental spectrum corresponding to helium droplets with an average size of 2700 atoms is shown in red ${ }^{194}$ The vertical lines indicate the D1 and D2 transitions of free $\mathrm{Ba}^{+}$.

provided in that figure demonstrates a good agreement between the two methods. Notice that such a good agreement is only possible when the 'solid' OT-DFT described in Sec. II C 1] is used. The conventional OT-DFT produces unphysically large pile-up of helium density around the cation. A similar level of agreement has been found for other cations. $\stackrel{3233}{3}$ The experimental realization of scolium was later achieved by using $\mathrm{Na}^{+}$rather than $\mathrm{Be}^{+} .202$

QMC and DFT calculations on $\mathrm{Rb}^{+}$and $\mathrm{Cs}^{+}$cations in helium droplets show that they are fully solvated and develop snowball structures. ${ }^{32 \mid 196}$ Based on DFT results, the first solvation shell around $\mathrm{Rb}^{+}$and $\mathrm{Cs}^{+}$hosts 19.2 and 21.4 atoms, respectively. These values are somewhat larger than those found experimentally 2031204 as well as by QMC calculations.195 On the contrary, the solvation structure around $\mathrm{a} \mathrm{Ba}^{+}$ion was found to be smooth without pronounced structure. This is in agreement with QMC calculations. 191 The difference in the solvation structures is a direct consequence of the much weaker interaction between $\mathrm{Ba}^{+}$ 


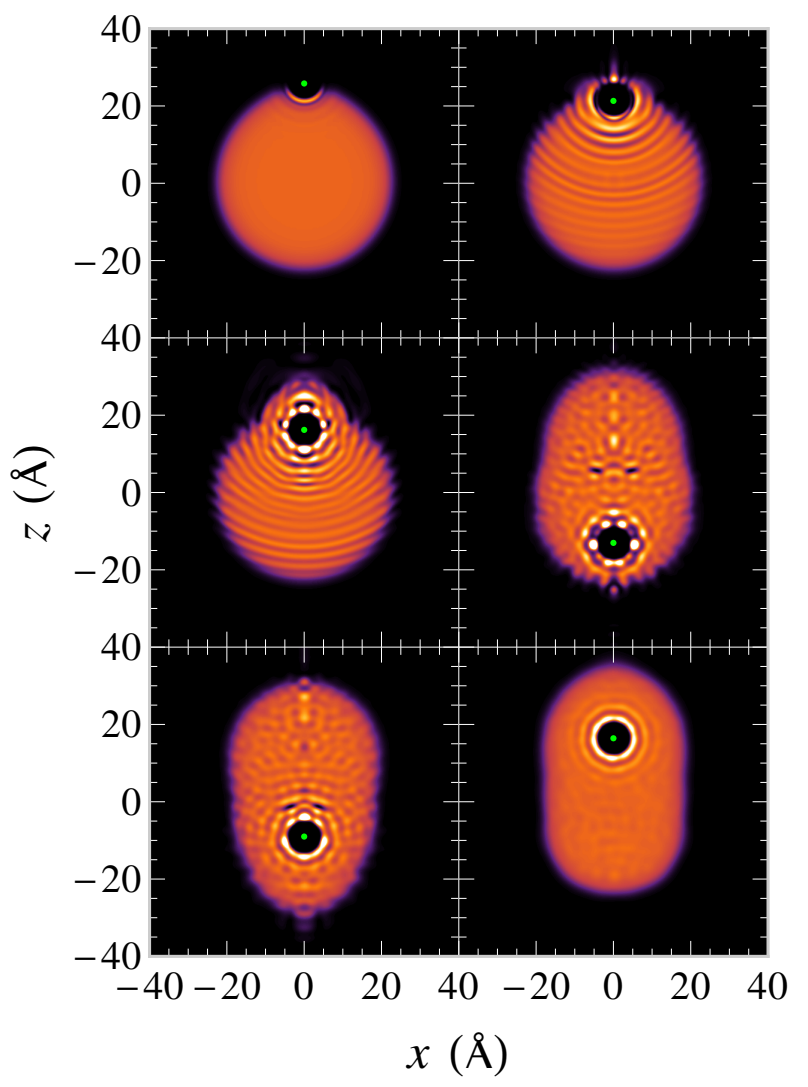

FIG. 24. Snapshots of the temporal evolution of $\mathrm{Ba}^{+} @{ }^{4} \mathrm{He}_{1000}$ after photoionisation of the neutral Ba atom located on the droplet surface. ${ }^{77}$ From top to bottom and left to right, the panels correspond to helium densities at times $t=0,8,14,47,60$ and 220 ps.

and helium compared to that of $\mathrm{Rb}^{+}$or $\mathrm{Cs}^{+}$with helium.

In general, cations are solvated in helium droplets because they exhibit very attractive interaction with helium. Based on this observation, experimentalists have studied the sinking of positive ions in ${ }^{4} \mathrm{He}$ droplets resulting from the ionisation of heliophobic alkali and alkaline earth metal atoms. ${ }^{194 / 202205}$ This activity has motivated the corresponding TDDFT simulations of the ion sinking process. ${ }^{3217786}$

Figure 23 shows the DFT time-resolved absorption spectrum of a $\mathrm{Ba}^{+} @^{4} \mathrm{He}_{1000} 6 \mathrm{p} \leftarrow$ $6 \mathrm{~s}$ transition after photoionisation of the surface-bound atom. The experimental data ${ }^{194}$ show a clear signature of the cation sinking process as the observed spectrum coincides with the corresponding absorption spectrum in the bulk liquid. ${ }^{206}$ DFT simulations of the ion 


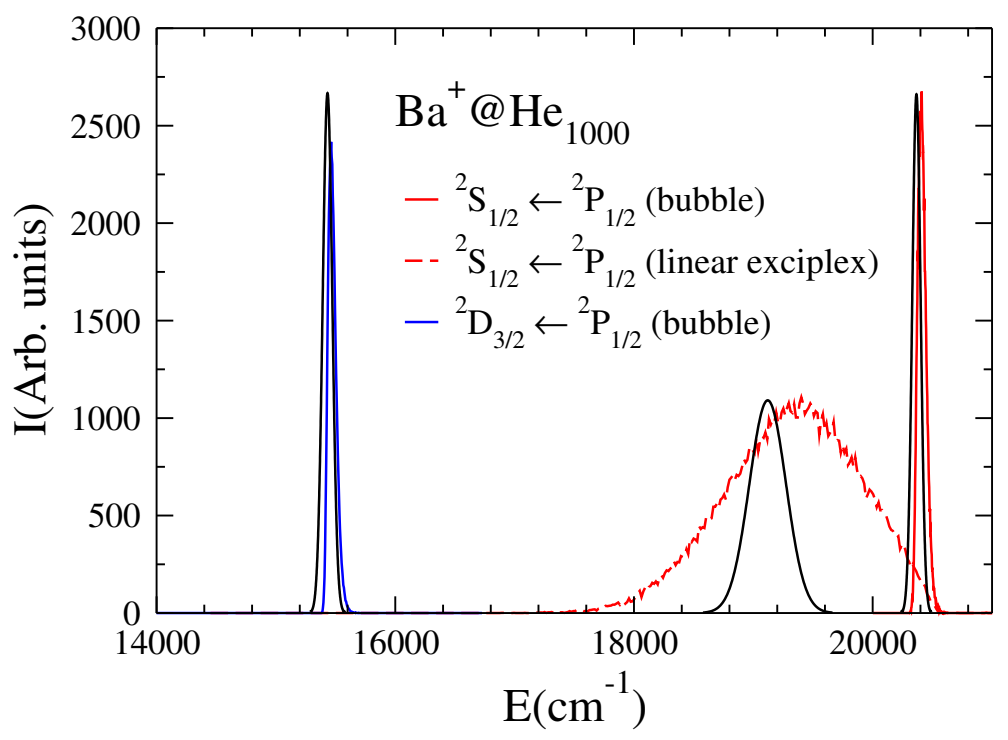

FIG. 25. Emission spectrum obtained from de-excitation of the relaxed ${ }^{2} \mathrm{P}_{1 / 2}$ state of a $\mathrm{Ba}^{+}$ cation in a ${ }^{4} \mathrm{He}_{1000}$ droplet. ${ }^{86}$ The gaussian lines shown in black are the experimental results. ${ }^{206}$

sinking into the droplet have revealed the nucleation of vortex rings as illustrated in Fig. 24. The snowball structure appears dynamically as bright spots around the cation during the initial solvation process, and wears out when the ion velocity decreases due to kinetic energy dissipation. The latter observation is in accordance with static DFT calculations. ${ }^{[32}$

In addition to the dynamic formation of the $\mathrm{Ba}^{+}$snowball and the time-resolved absorption spectra of this cation, the most interesting outcome of the calculations is the formation of a vortex ring at the equator of the $\mathrm{Ba}^{+}$solvation structure after about $13 \mathrm{ps}$. This vortex ring slips around the ion and eventually detaches at $24 \mathrm{ps}$. The cross-section of the vortex ring can be readily identified from the two dark spots behind the ion bubble at 47 ps as shown in Fig. 24. Calculation of the circulation around the core yields a value of unity in units of $h / m_{4}$. Eventually, the vortex ring is destroyed by colliding with the ion bubble.

Alkali metal atoms reside on the droplet surface like the heavy alkaline earth atoms, but the interaction of the corresponding ions with helium is strongly attractive. Hence their dynamics upon ionisation is expected to be similar to that of barium. This motivated the TDDFT study of $\mathrm{Rb}^{+}$and $\mathrm{Cs}^{+}$cations produced by the photoionisation of the neutral atom on the droplet surface. ${ }^{32}$ Surprisingly, in neither case did the sinking process any vortex 
rings. This was attributed to a subtle effect overlooked in the previous studies. Comparison of the initial surface solvation structures of $\mathrm{Ba} v \mathrm{vs}$. $\mathrm{Rb}$ or $\mathrm{Cs}$ shows that the latter species are located in a shallower dimple such that the ion is farther away from the droplet surface. Upon ionisation, $\mathrm{Rb}^{+}$and $\mathrm{Cs}^{+}$pull the lighter helium atoms on the surface towards them and the resulting structure 'floats' on the droplet. This screens the interaction of the cation with the rest of the nearby He atoms in the droplet and, as a consequence, their sinking velocity is lower than for $\mathrm{Ba}^{+}$. Moreover, the sinking process requires a much larger droplet to take place; for example, $\mathrm{Cs}^{+}$did sink inside a ${ }^{4} \mathrm{He}_{2000}$ droplet but not in ${ }^{4} \mathrm{He}_{1000}$. In the latter case, it was actually expelled from the droplet as a charged minicluster. Both cations were found to sink when the neutral parent atom was located on the liquid free surface that might locally represent a very large droplet. Interestingly, vortex loops (i.e. vortex segments that start and end on the droplet surface) were nucleated by the appearance of local distortions in the droplet surface during the sinking process .32

The desolvation dynamics following $6 \mathrm{p} \leftarrow 6$ s excitation of $\mathrm{Ba}^{+}$in helium droplets was further investigated in a joint experimental and theoretical work ${ }^{\sqrt{86}}$ The experiment showed that the desolvation process yielded mainly bare $\mathrm{Ba}^{+}$and $\mathrm{Ba}^{+} \mathrm{He}_{n}$ exciplexes with $n=1$ and 2. In terms of TDDFT simulations, this process is similar to the evolution of photoexcited Ag atoms in helium droplets. ${ }^{44}$ As shown in Fig. 25, the calculations reproduced the main features of the experimental $\mathrm{Ba}^{+}$emission spectrum${ }^{206}$ and, furthermore, they demonstrated the dynamical formation of exciplexes. These linear and ring geometry $\mathrm{Ba}^{+}-\mathrm{He}_{n}$ exciplexes were previously found by QMC, ${ }^{87}$ where the experimentally observed line at $19120 \mathrm{~cm}^{-1}$ was assigned to the de-excitation of the $\mathrm{Ba}^{+}\left({ }^{2} \Pi_{1 / 2}\right) \mathrm{He}_{2}$ linear exciplex.

Despite the above achievements, the DFT approach did not yield the detachment of excited $\mathrm{Ba}^{+}$ions as found in the experiments. The origin of this discrepancy was extensively discussed in Ref. 86 and the ejection mechanism of photoexcited $\mathrm{Ba}^{+}$from helium droplets is still an open question. Elucidating this issue would not only require additional experimental data (e.g. state distribution of the desolvated $\mathrm{Ba}^{+}$ions), but also an improved theoretical model which includes non-adiabatic transitions between electronic states. $\stackrel{110}{ }$ Indeed, nonradiative relaxation of the excited states to the ${ }^{2} \mathrm{D}$ state or to the ${ }^{2} \mathrm{~S}$ ground state could deposit a sufficient amount of energy into the system to eject $\mathrm{Ba}^{+}$and/or blow up the droplet. So far, this has not been explicitly shown and this proposition should just be viewed as an 'educated guess'. 


\section{H. Intrinsic helium impurities}

Ionisation of superfluid helium and subsequent charge recombination leads to the generation of intrinsic singlet and triplet state $\mathrm{He}^{*}$ atoms and $\mathrm{He}_{2}^{*}$ excimers in the liquid $207 / 208$ While the singlet states rapidly decay to the electronic ground state through radiative processes, the triplet $\mathrm{He}^{*}(1 \mathrm{~s} 2 \mathrm{~s})$ and $\mathrm{He}_{2}^{*}\left({ }^{3} \mathrm{a}\right)$ states are metastable due to the lack of spin-orbit coupling. For this reason, most experimental work has concentrated on employing the triplet species to study the response of the surrounding bulk liquid.2091210

To study the solvation of triplet He* species in superfluid helium by OT-DFT, ab initio electronic structure calculations have been conducted to map out the $\mathrm{He}^{*}-\mathrm{He}(1 \mathrm{~s})$ interaction in its various electronic states. 211214 The interaction of these and higher excited triplet species with ground state He atoms was found mostly repulsive, i.e. they are heliophobic and form bubble states in superfluid helium. However, nodal planes in excited Rydberg state orbitals can create close range attractive pockets in which helium atoms can accumulate.215 The static solvation structure around $\mathrm{He}^{*}$ was obtained using OT-DFT with He* treated quantum mechanically due to its light mass, see e.g. Eq. (36).

Since the obtained bubble interfaces have appreciable width, the exact meaning of the bubble radius must be unambiguously defined. For a spherical solvation bubble in the bulk liquid, the interface average radius $R_{b}$ can be obtained from

$$
R_{b}=\left[\frac{3}{4 \pi} \int d \mathbf{r}\left(1-\frac{\rho(\mathbf{r})}{\rho_{0}}\right)\right]^{1 / 3}
$$

Note that this form is only applicable to bubble structures whereas no clear definition for snowball-type solvation cavities can be given. Using Eq. (64), bubble radii in the range of 6-12 $\AA$ have been obtained for $\mathrm{He}^{*}(1 \mathrm{~s} 2 \mathrm{~s})$ and $\mathrm{He}^{*}(1 \mathrm{~s} 3 \mathrm{~s})$ depending on the pressure. $212 \mid 213$ Since the external potentials have essentially no binding, the interfaces appear smooth with the exception of weak oscillatory structures arising from the correlated nature of the liquid.

To establish a comparison with existing experimental data, absorption (i.e. He* $1 \mathrm{~s} 2 \mathrm{p})$ $\left.\leftarrow \operatorname{He}^{*}(1 \mathrm{~s} 2 \mathrm{~s})\right)$ and fluorescence (i.e. $\left.\mathrm{He}^{*}(1 \mathrm{~s} 3 \mathrm{~s}) \rightarrow \mathrm{He}^{*}(1 \mathrm{~s} 2 \mathrm{p})\right)$ line shapes were calculated by TDDFT. 212213 Since the technique for evaluating spectral lineshape is different from that presented in Sec. IV C, a short description is provided here.

Given the static liquid density profile around the initial state, time evolution of the liquid in the final state can be used to determine the first order polarization at excitation angular 
frequency $\omega$ as 100

$$
P^{(1)}(t) \propto \int_{0}^{t} d t^{\prime} \exp \left(-\frac{i}{\hbar} \int_{t^{\prime}}^{t} d t^{\prime \prime} \Delta E\left(t^{\prime \prime}\right)-i \omega t^{\prime}\right)+\text { C.C. }
$$

where C.C. stands for complex conjugate of the preceding term and $\Delta E$ is the energy difference between the two electronic states. For example, for an absorption process

$$
\begin{aligned}
\Delta E(t)= & \iint d \mathbf{r} d \mathbf{r}^{\prime} \rho_{u}^{\prime}\left(\mathbf{r}^{\prime}, t\right) V_{u}\left(\left|\mathbf{r}-\mathbf{r}^{\prime}\right|\right) \rho_{u}(\mathbf{r}, t) \\
& -\iint d \mathbf{r} d \mathbf{r}^{\prime} \rho_{l}^{\prime}\left(\mathbf{r}^{\prime}, 0\right) V_{l}\left(\left|\mathbf{r}-\mathbf{r}^{\prime}\right|\right) \rho_{l}(\mathbf{r}, 0)
\end{aligned}
$$

where $\rho_{l}(\mathbf{r}, 0)$ and $\rho_{u}(\mathbf{r}, t)$ represent the densities for the initial and final states, respectively. The primed quantities refer to the probability density of the impurity that is treated quantum mechanically. Note that for fluorescence the roles of the upper and lower levels are reversed, the initial upper level contribution is time-independent and the dynamics takes place on the lower level potential. To include dephasing in Eq. (65), the polarization can be multiplied by a phenomenological exponential decay, $\bar{P}^{(1)}(t)=e^{-t / \tau} P^{(1)}(t)$, where $\tau$ is the dephasing time constant. During this time, the spectrum is sensitive to the impurity-helium bath interaction. The linear absorption or fluorescence spectrum is finally obtained by Fourier transforming the polarization provided by Eq. 65). A comparison between experimental $2 \mathrm{p} \leftarrow 2 \mathrm{~s}$ absorption line shift as a function of external pressure and the OT-DFT results employing this method is shown in Fig. 26. ${ }^{213}$ The best match with experiments is obtained with $\tau=150$ fs. Since the response time associated with bubble breathing and interface curvature dynamics is longer than this dephasing time, the resulting absorption spectra appear broad and exhibit no additional structure.

The above line shape model has also been employed to calculate $\mathrm{He}^{*}(1 \mathrm{~s} 3 \mathrm{~s}) \rightarrow(1 \mathrm{~s} 2 \mathrm{p})$ and $\mathrm{He}_{2}^{*}{ }^{3} \mathrm{~d} \rightarrow{ }^{3}$ a fluorescence line shifts as a function of pressure. ${ }^{212 \mid 214}$ In general, the calculations show slightly larger blue shifts than the experimental data, which may be related to the higher temperature in the experiments vs. calculations or to the accuracy of the used $\mathrm{He}^{*}-\mathrm{He}$ and $\mathrm{He}_{2}^{*}$-He pair potentials. Note that the experimental line shifts are sensitive to energy differences of just a couple of $\mathrm{cm}^{-1}$ far away from the impurity.

Finally, we mention a time-dependent OT-DFT calculation modelling the superfluid dynamics following a two-photon excitation of the $\mathrm{He}_{2}^{*}$ excimer from the ${ }^{3}$ a to the ${ }^{3} \mathrm{~d}$ state..$^{218}$ These calculations, which were carried out in 1D spherical coordinates, were motivated by earlier optical pump-probe measurements that determined the bubble breathing period 


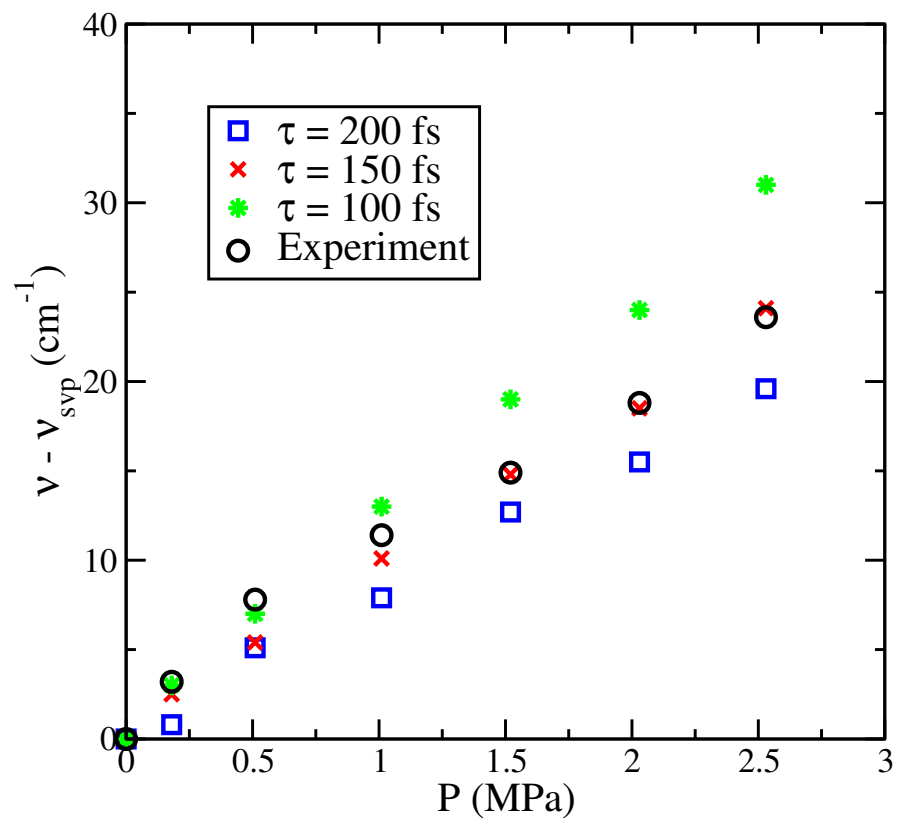

FIG. 26. Pressure induced shift for the $\mathrm{He}^{*}(1 \mathrm{~s} 2 \mathrm{p}) \leftarrow(1 \mathrm{~s} 2 \mathrm{~s})$ absorption line in superfluid helium. 213 Comparison between the calculated line shifts (OT-DFT and Eq. (65)) for selected values of the dephasing time constant $\tau$ and the experimental data obtained at $1.6 \mathrm{~K}$ temperature.216 217

around the ${ }^{3} \mathrm{~d}$ state as a function of $P$ and $T \stackrel{210}{2}$ The period was observed to track the bulk liquid viscosity and reached $c a .150 \mathrm{ps}$ at the lowest measured temperature of $1.4 \mathrm{~K}$. The normal fluid fraction at this $T$ is only 0.08 , which implies that the viscous contribution to the breathing period should be very small. While the OT-DFT calculations carried out in Ref. 218 did not include this viscous response, bubble breathing periods in the range of 50 to 120 ps were obtained depending on the $\mathrm{He}_{2}^{*}$-He potential employed. Two open questions still remain regarding this system: 1) inclusion of the viscous response [see Eq. 699]; and 2) accurate calculation of the long-range $\mathrm{He}_{2}^{*}\left({ }^{3} \mathrm{~d}\right)$-He interaction potential. The latter may also require inclusion of many-body corrections beyond the pair potential approximation as the ${ }^{3} \mathrm{~d}$ Rydberg orbital is somewhat compressible. 211 


\section{Translational motion of ions below the Landau critical velocity}

In addition to the above mentioned solvation dynamics of ions in superfluid helium, the hydrodynamic response of the surrounding liquid due to translational motion of various ions has been studied by OT-DFT. ${ }^{33219}$ At $T=0$, provided that the ion velocity remains well below the Landau critical value, dissipation of energy can only take place through the emission of sound when the ion accelerates or decelerates in the liquid. In the presence of thermal excitations (i.e. thermal phonons and rotons), the viscous drag force also opposes the ion motion. The most important experimentally accessible parameters that are sensitive to this dissipative liquid response are the ion hydrodynamic mass $\left(m_{a d d}\right)$ and the ion mobility $(\mu)$. The former quantity corresponds to the difference between the bare ion mass in vacuum and its effective mass in the liquid whereas the ion mobility is determined by the ion steadystate velocity in the liquid.2

Hydrodynamic added masses for several halogen anions ${ }^{33}$ as well as bare positive (i.e. $\mathrm{He}_{3}^{+}$) and negative charges ${ }^{219}$ in superfluid ${ }^{4} \mathrm{He}$ have been calculated by OT-DFT. In the latter work, the ion mass was computed by imaginary-time OT-DFT in the co-moving reference frame (see also Sec. VF)

$$
\left[\hat{H}-v_{0, z} \hat{P}_{z}\right] \Psi(\mathbf{r})=\mu \Psi(\mathbf{r})
$$

where $v_{0, z}$ is the constrained liquid velocity along the $z$-axis and $\hat{P}_{z}$ is the $z$-component of the momentum operator. An estimate for the added mass during imaginary-time iterations can be computed from

$$
\frac{m_{a d d}}{m_{4}}=\frac{1}{v_{0, z}} \int d \mathbf{r} \rho(\mathbf{r}) v_{z}(\mathbf{r})
$$

where $v_{z}$ is the $z$-component of the liquid velocity $\mathbf{v}(\mathbf{r})=\mathbf{j}(\mathbf{r}) / \rho(\mathbf{r})$. This model yields results consistent with the available experimental information (i.e. positive and negative

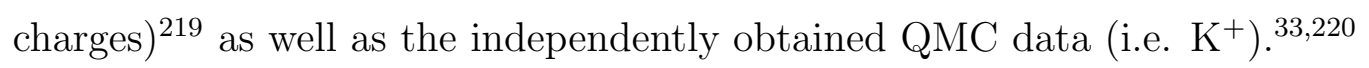

In ion mobility experiments, an external electric field accelerates the ion until the electrostatic and hydrodynamic drag forces cancel. The resulting steady-state velocity $v_{z}$ is directly related to the ion mobility, $\mu=e v_{z} / F_{z}$, where $F_{z}$ is the electrostatic force acting on the ion. In a liquid at $T \neq 0$, the drag force arises from collisions with thermal excitations, whereas in the limit of $0 \mathrm{~K}$ the ion could in principle accelerate until the critical value for the creation of vorticity or turbulence is reached (see Sec. VF for the case of an electron). 
To help comparison with the available experimental mobility data, ${ }^{2}$ the standard OTDFT functional must be extended to include the liquid viscous response. At temperatures higher than $1.4 \mathrm{~K}$, the roton density is sufficiently high for a continuum-based model to be applicable. The viscous response term from the Navier-Stokes equation can be adapted to DFT by employing the Madelung transformation, 221 see also Sec. IV B. This gives the following equation for the associated non-linear potential, $V_{N S}=V_{N S}[\rho, \mathbf{v}]$,

$$
\nabla^{2} V_{N S}=-\nabla \cdot\left\{\frac{1}{\rho}\left[\eta\left(\nabla \mathbf{v}+(\nabla \mathbf{v})^{\mathrm{T}}-\frac{2}{3}(\nabla \cdot \mathbf{v}) \mathbf{1}\right)\right]\right\}
$$

where $\eta=\eta(\rho, T)$ is the liquid shear viscosity, 1 denotes the unit tensor, and superscript $\mathrm{T}$ denotes matrix transpose. Note that this form allows for both liquid compression $(\nabla \cdot \mathbf{v} \neq 0)$ and rotation $(\nabla \times \mathbf{v} \neq 0)$ as well as spatial variation of the viscosity. The discrete form of this equation reduces to the Poisson problem, which can be efficiently solved in Fourier space using standard techniques.

An additional complication arises from the presence of a wide gas-liquid interface surrounding most ions in superfluid helium. To obtain agreement with the experimental electron mobility data, the shear viscosity in this region must be modified from the bulk value, $\eta(\rho(\mathbf{r}), T)=\left[\rho(\mathbf{r}) / \rho_{0}(T)\right]^{\alpha(T)} \eta_{0}(T) ! 221$ where $\alpha(T)$ determines the spatial variation of the viscosity across the interface and $\eta_{0}$ is the bulk shear viscosity.

The steady-state liquid flow solution around the ion can be obtained by including Eq. (69) in the OT functional and propagating the system in imaginary-time according to the velocity constraint of Eq. (67). Under this condition, the electrostatic and drag forces cancel out and the ion mobility can be evaluated. The hydrodynamic drag on the ion can simply be obtained by calculating the force due to the surrounding liquid for classical impurities (see Eq. (47)). If the impurity (e.g. electron) is treated quantum mechanically, integration over the impurity coordinate must also be included. This model was shown to reproduce the known electron mobility data between $1.4 \mathrm{~K}$ and the lambda point along the saturated vapor pressure line. ${ }^{221}$ As an illustration, Fig. 27 shows the results for an electron moving in superfluid helium at $2.1 \mathrm{~K}$. 


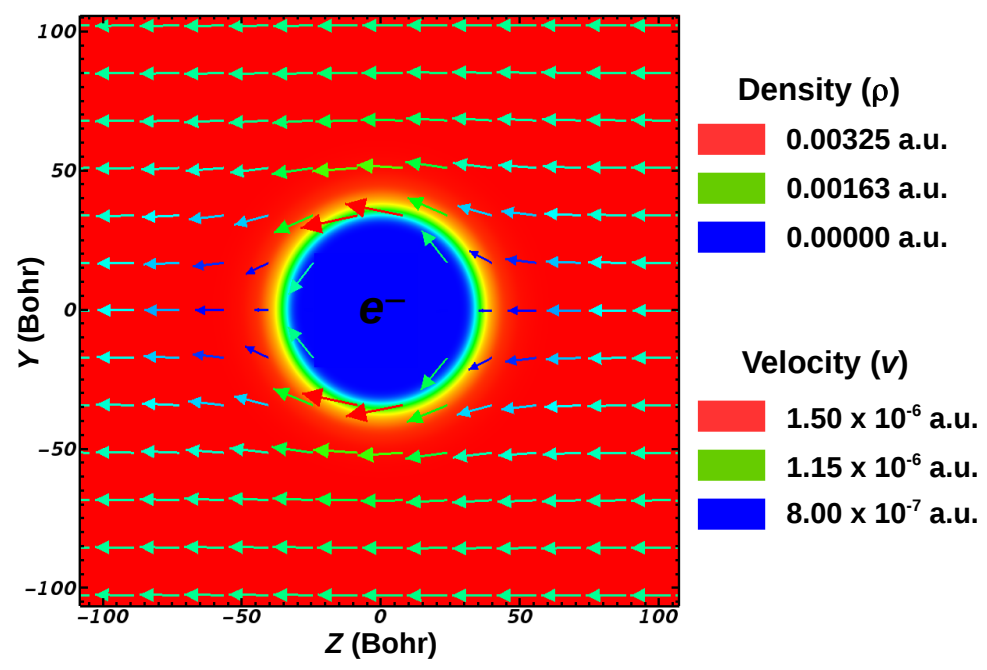

FIG. 27. Steady-state helium density contours $(\rho)$ and velocity field $(v)$ around an electron moving in superfluid helium at $T=2.1 \mathrm{~K}$. The velocity component shown along the $z$-axis was shifted by $v_{0}=-2.3 \mathrm{~m} / \mathrm{s} \cdot 221$

\section{J. Critical Landau velocity in small ${ }^{4} \mathrm{He}$ droplets}

Many properties of helium nanodroplets have been characterised during the last two decades by using solvated molecules as spectroscopic probes. In particular, vibrational and rotational spectroscopy of solvated carbonyl sulfide (OCS) provided evidence for microscopic superfluidity in these finite size systems. $\left[\frac{6[155]}{}\right.$ However, this raises the question to what extent can microscopic superfluidity be related to the frictionless flow of superfluid helium.

The first point considered was the existence of Landau critical velocity in helium nanodroplets, which are microscopic objects of only $10^{3}-10^{6}$ atoms, and the possibility of using atoms or molecules as probes for it. This was the starting point of a joint experimental and theoretical $\operatorname{search}^{30}$ for the existence of a limiting velocity for species ejected from helium droplets.

The following scheme was designed for that purpose. A probe atom or molecule, initially located in the bulk portion of the droplet, was suddenly optically excited to an electronic state with repulsive interaction with helium. As a consequence, the probe is accelerated and 


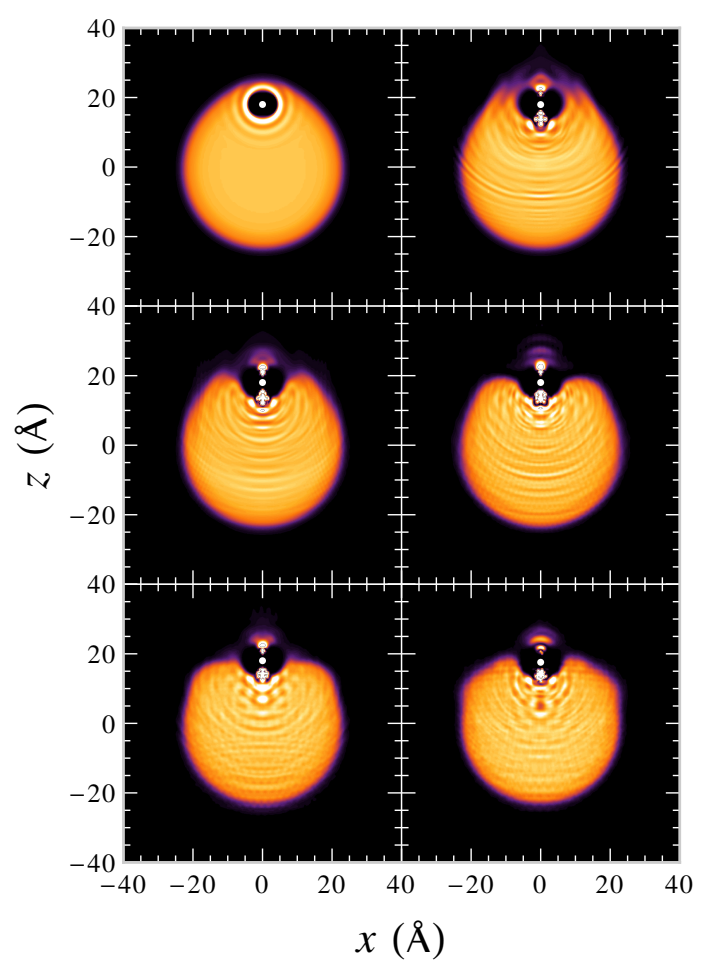

FIG. 28. Dynamic evolution of an $\mathrm{Ag} @{ }^{4} \mathrm{He}_{1000}$ complex upon sudden excitation of $\mathrm{Ag}$ to the ${ }^{2} \mathrm{P}_{3 / 2}$ state displaying the appearance of a linear $\mathrm{AgHe}_{2}$ exciplex on the droplet surface. The $\mathrm{Ag}$ atom is initially at rest $18 \AA$ off the center of the droplet. Snapshots are shown every 4 ps starting from the top left frame. $\stackrel{44}{ }$

ejected from the droplet. If there is a critical velocity, the probe cannot accelerate beyond it and this will be reflected in its final velocity. Experimental measurements of the velocity distributions of atoms/molecules ejected from various size helium droplets have revealed the existence of a critical velocity threshold even for droplets consisting of a thousand helium atoms. In particular, ${ }^{2} \mathrm{P}_{1 / 2}$ excitation of $\mathrm{Ag}$ leads to its ejection with a velocity distribution peaking around $55 \mathrm{~m} / \mathrm{s}$. A similar, although not identical, velocity distribution was obtained upon excitation to the ${ }^{2} \mathrm{P}_{3 / 2}$ state, where the ejected species was either Ag or AgHe. DFT simulations on the dynamic evolution of this system ${ }^{44 \mid 86}$ confirmed these findings and provided additional microscopic details of the process. In particular, running the simulation for several tens of picoseconds was sufficient to observe the AgHe exciplex formation as shown 
in Fig. 28,

\section{K. Rotational superfluidity}

Superfluidity of helium droplets has been extensively studied by both experiments and theory. ${ }^{19 \mid 222[223}$ As discussed earlier, previous experimental work has employed molecular probes to interrogate the droplet response to both radial and rotational excitation. $\stackrel{4224}{ }$ While these experiments have demonstrated the presence of the characteristic roton energy gap for larger droplets (i.e. the Landau criterion for superfluidity), microwave spectroscopy experiments indicated that non-classical behavior already takes place in molecule-helium clusters with less than ten He atoms. ${ }^{224}$ In these experiments, the rotational constant $B$ (proportional to the inverse of the moment of inertia) was determined as a function of the number of helium atoms in the cluster. $B$ was observed to initially decrease with $N_{4}$ as expected for a classical rotor, but then it started increasing again from a given size on, which depended on the probe, $225+227$ as shown in Fig. 29. This turning point has been interpreted as the onset of superfluidity in small helium droplets $\left(N_{4}<20\right) \stackrel{224}{[}$ It seemed to contradict the original Laudau criterion for superfluidity since previous QMC calculations had shown ${ }^{228 \mid 229}$ that there was no roton energy gap in droplets with less than ca. $64{ }^{4} \mathrm{He}$ atoms.

Recent OT-DFT calculations employing the rotational constraint of Eq. (31) have resolved this discrepancy by identifying the quantum mechanical origin of the non-classical reduction in rotational friction. 219 The only input to this model is the probe molecule-He interaction, i.e. the external potential for OT-DFT, taken from ab initio electronic structure calculations. The rotationally constrained OT-DFT equation was solved by the ITM, which yielded the stationary order parameter $\Psi$ with the associated liquid density and velocity field rotating with the molecule. The added moment of inertia for the rotor $\left(I_{a d d}\right)$, which is equivalent to $m_{a d d}$ for translation motion, can be computed from

$$
I_{a d d}=\left\langle\Psi\left|L_{z}\right| \Psi\right\rangle / \omega
$$

where $\hat{L}_{z}$ is the $z$-component of the liquid angular momentum operator and $\omega$ is the frequency of rotation - typically less than $1 \mathrm{GHz}$; see Eq. (31). The effective rotational constant of 
the molecule in superfluid helium, $B_{\text {eff }}$, is then given by

$$
B_{e f f}=\frac{\hbar}{4 \pi c\left(I_{g a s}+I_{a d d}\right)}
$$

where $I_{\text {gas }}$ is the moment of inertia of the molecule in the gas phase.

Four different probe molecules, which can be classified as 'heavy' or 'light' rotors based on their gas phase moments of inertia, were studied ${ }^{219}$ (see Fig. 29). The results showed that the experimentally observed turning points in the $B\left(N_{4}\right)$ curve correlated with helium coverage of the probe molecule. When a connected path of helium forms around the molecule, helium attempts to remain irrotational by introducing negative angular momentum to decouple from the rotational motion. This explains why the position of the turning point depends on the probe molecule itself. In addition, this turning point correlates with the appearance of a continuous helium coverage around the probe rather than the completion of the first solvation shell. Secondary oscillations in $B\left(N_{4}\right)$ were related to the complete coverage of the subsequent helium layers.

The appearance of global phase coherence around the probe molecule produces a Landautype energy gap between the droplet rotational ground and first excited states. This gap plays a similar role for rotational motion as the Landau roton gap in traditional superfluidity. When analysed in the co-rotating frame of reference, the transition bears similarities to the Mott-1D superfluid quantum phase transition. ${ }^{230}$ In order to distinguish this phenomenon from the traditional translational superfluidity, we refer to it as rotational superfluidity! 219

\section{Interaction of impurities with vortex lines}

Much of what is known on vortices in helium has been drawn using ions and electrons as probes in experiments. In this section we present results for ions, electrons, and neutral impurities obtained by static DFT calculations and leave the discussion of dynamical capture of impurities by vortices in helium droplets 231 to Sec. VO, The reader should note the different sign convention for the binding energy of an impurity to the vortex line used in the following Secs. VL1 and VL2, We have kept the convention used in the original papers. 


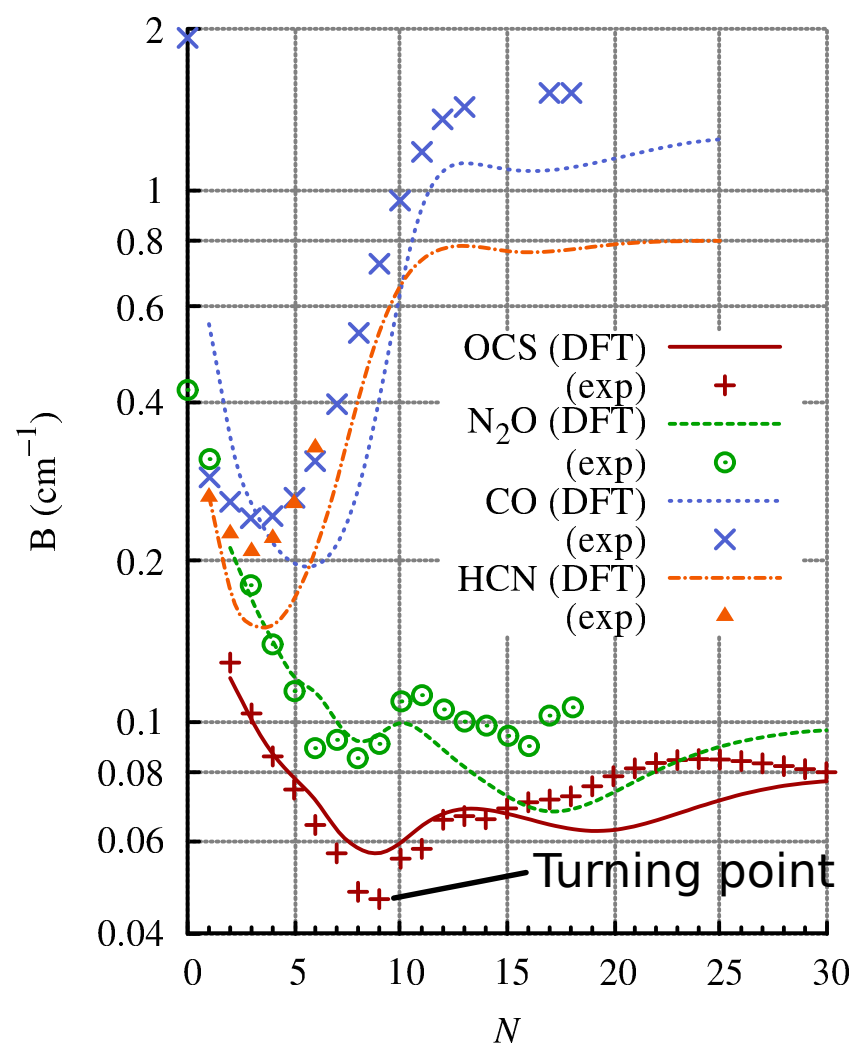

FIG. 29. Experimental (exp) and calculated (DFT) effective rotational constants as a function of helium droplet size. The position of the non-classical turning point for OCS is indicated by a line .219

\section{Electrons}

DFT calculations can provide detailed information about electron trapping on quantised vortex lines. ${ }^{69}$ The degenerate electronic states (e.g. p, d, f ...) of the bubble split if the bubble becomes non-spherical. For this reason, when the electron bubble becomes trapped on a vortex line, the resulting symmetry breaking leads to the splitting of the energy levels. Provided that this splitting is large enough, it could be observed by absorption spectroscopy.

The absorption spectrum of the electron bubble can be obtained from the dipole strength 
function $S(\omega)$

$$
S(\omega)=\sum_{n \neq 0}|\langle n|\mathbf{r}| 0\rangle|^{2} \delta\left(\omega-\omega_{n 0}\right)
$$

where $|0\rangle$ and $|n\rangle$ correspond to the ground and excited states of the electron bubble, respectively. The infrared absorption spectrum of the e-bubble from DFT calculations is shown in Fig. 30 in the range of $1 \mathrm{~s}-1 \mathrm{p}$ and $1 \mathrm{~s}-2 \mathrm{p}$ transitions. The function $S(\omega)$ displays peaks centered at the absorption energies $\omega_{n 0}$. Furthermore, the figure shows that the effect of vortex trapping on the absorption spectrum is very small, especially for the $1 \mathrm{~s}-1 \mathrm{p}$ transition. Slightly larger changes are observed for the $1 \mathrm{~s}-2 \mathrm{p}$ transition, which originates from the more pronounced penetration of the $2 \mathrm{p}$ electron wave function into the liquid. However, this transition is much weaker than the $1 \mathrm{~s}-1 \mathrm{p}$ one. Based on these results, it can be concluded that the infrared absorption spectrum of the e-bubble is not very sensitive to the possible vortex trapping and hence, it is not generally suitable for detecting vorticity.

The 1p-1s emission energy has been calculated for both free and vortex trapped ebubbles. ${ }^{113}$ This calculation assumes that the radiative lifetime of the excited state is longer than the time required for the liquid to equilibrate around the electron. Experimental data indicate that the radiative lifetime is some tens of nanoseconds 232 or even tens of microseconds when calculated directly from the transition dipole moment ${ }^{180}$ Based on the simulations, the equilibrium geometry around $1 \mathrm{p}$ is reached after several hundreds of picoseconds.

The most important quantity that can be extracted from the calculations is the binding energy of the e-bubble to the vortex line. This can be obtained from the grand potential per unit volume, $\Omega \equiv\left(F-\mu_{4} N_{4}\right) / \mathcal{V}$, for e-bubble configurations with and without a vortex line at the same temperature and pressure:

$$
B_{e-V}=\left[\Omega_{e}-\Omega\right]-\left[\Omega_{e+V}-\Omega_{V}\right]
$$

where the subscripts refer to vortex-free e-bubble $(e)$, bulk liquid (none), e-bubble attached to a vortex line $(e+V)$, and vortex line alone $(V)$. Since all the terms above are evaluated under the same thermodynamic conditions, their chemical potential $\mu_{4}$ and the saturation liquid density are identical. The above expression is therefore well-defined and independent of the simulation box volume. Alternatively, one may use the substitution energy introduced in Sec. IIIB, which differs from the value of Eq. (73) by ca. $10 \%$. The calculations yield the electron binding energy as $B_{e-V}=104.5 \mathrm{~K}$ at $P=T=0$, which shows that trapping of electron bubbles on vortex lines is energetically very favored. A value of $109 \mathrm{~K}$ was found in 


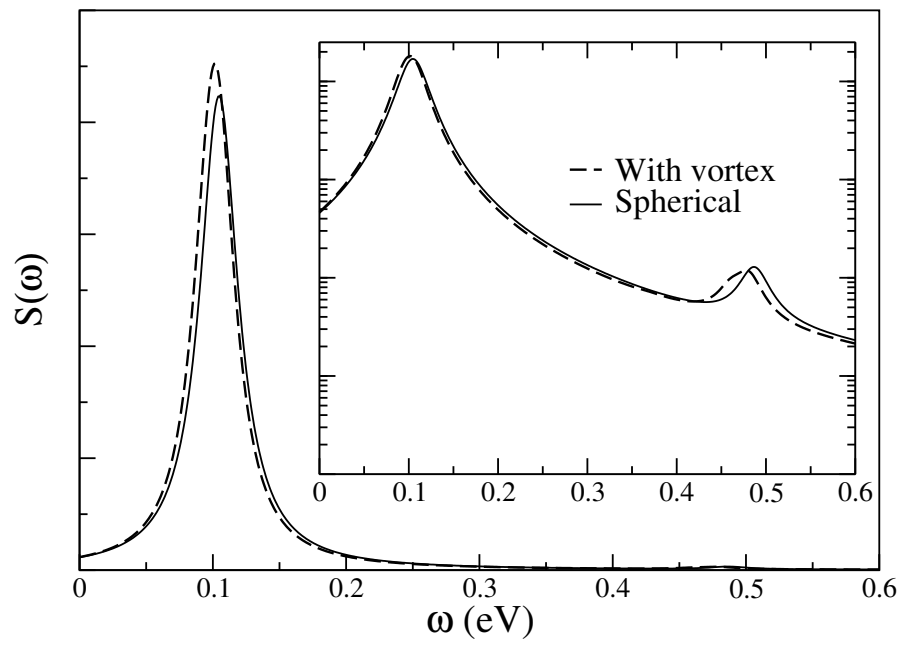

FIG. 30. Dipole strength $S(\omega)$ (arbitrary scale) at $P=T=0$ for a spherical electron bubble (solid line) and for an electron bubble attached to a linear vortex (dashed line). The inset shows $S(\omega)$ in logarithmic scale.113

Ref. 43; the difference between the two values is attributed to the different electron-helium interactions used.

The attractive interaction between an electron and a vortex line can be understood in terms of the loss of kinetic energy due to the liquid displaced by the approaching electron bubble (i.e. the classical Bernoulli force) $!^{2}$ The experimentally obtained values for electron binding to vortex lines vary between 55 and $59 \mathrm{~K}$ at $T=1.6 \mathrm{~K}, \frac{742331234}{2}$ wich is in clear disagreement with the value obtained from OT-DFT calculations at $T=0$. Since this rather deep binding value is consistent with the large radius of the electron bubble, $181+184$ the difference has been attributed to the $T$ dependence of the vortex core parameter and local thermal deformations of the vortex line rather than to a deficiency in the OT-DFTbased model itself. ${ }^{43|69| 235}$ Furthermore, the same OT-DFT model is able to reproduce the experimental binding energy of positive charges (i.e. $\mathrm{He}_{3}^{+}$, Ref. 236) to vortex lines: $16 \mathrm{~K}$ (calculated at $0 \mathrm{~K}$ ) vs. $17.5 \mathrm{~K}$ (experiment at $0.3 \mathrm{~K}$ ) $\stackrel{43}{ }$ Once the thermal effects for the electron are taken into account, the OT-DFT binding energy is lowered down to $61 \mathrm{~K}$, which is very close to the experimental estimates. $\stackrel{43}{~ T h u s, ~ v o r t e x ~ l i n e s ~ p r e s e n t ~ v e r y ~ d e e p ~ t r a p s ~ f o r ~}$ electrons in superfluid helium. 


\begin{tabular}{|c|c|c|c|c|}
\hline Impurity & $\begin{array}{l}R \\
(\AA)\end{array}$ & $\begin{array}{l}a_{F} \\
(\AA)\end{array}$ & $\begin{array}{l}\text { OT-DFT } V_{\text {im-vortex }} \\
(\mathrm{K})\end{array}$ & $\begin{array}{c}\text { Exp. } V_{\text {im-vortex }} \\
(\mathrm{K})\end{array}$ \\
\hline $\mathrm{H}_{2}\left(X^{1} \Sigma_{g}\right)$ & 3.1 & 0.38 & -9.4 & - \\
\hline $\operatorname{Ag}_{2}\left(X^{1} \Sigma_{g}\right)$ & 3.9 & 0.51 & -10.9 & - \\
\hline $\mathrm{Cu}_{2}\left(X^{1} \Sigma_{g}\right)$ & 4.0 & 0.52 & -11.4 & - \\
\hline $\operatorname{Ag}\left({ }^{2} S\right)$ & 4.4 & 0.63 & -12.0 & - \\
\hline $\mathrm{Cu}\left({ }^{2} S\right)$ & 4.5 & 0.65 & -12.5 & - \\
\hline $\mathrm{He}_{3}^{+}\left(X^{2} \Sigma_{g}\right)$ & $\approx 5.7$ & $\approx 0.75$ & -16.0 & -17.5 at $0.28-0.6 \mathrm{~K}$, Refs. 243 and 244 \\
\hline $\mathrm{Li}\left({ }^{2} S\right)$ & 6.8 & 0.75 & -21.5 & - \\
\hline $\mathrm{He}^{*}\left(2 s^{3} S\right)$ & 7.1 & 0.77 & -22.6 & - \\
\hline $\mathrm{He}_{2}^{*}\left(a^{3} \Sigma_{u}\right)$ & 8.6 & 0.80 & -29.8 & - \\
\hline$e^{-}(1 s)$ & 22.2 & 0.76 & $-109^{\mathrm{a}}$ & -55 to -59 at $1.6 \mathrm{~K}$, Refs. 233 and 234 \\
\hline
\end{tabular}

TABLE III. Summary of the impurity-vortex interaction parameters at $P=T=0$ based on Eq. (75). $R$ represents the classical bubble radius for the impurity, $a_{F}$ is the healing length, and $V_{\text {im-vortex }}(0)$ denotes the total binding energy $\stackrel{43}{ }$ a See discussion in Sec. VF regarding the value for the electron.

\section{Atomic and molecular impurities}

Just as electrons trap on vortex lines, any impurity should be attracted towards them due to the Bernoulli force. Given a sufficiently high concentration of neutral impurities in the liquid, they can accumulate on vortex lines as a consequence of this attraction. Subsequent diffusion along the vortex line may then lead to the assembly of nanowires, which are reminiscent of the original vortex line geometry. The final products from this process have been observed in metal doped bulk superfluid helium $237 \underline{239}$ and superfluid helium droplets. $.240 \mid 242]$ One of the main factors influencing the initial stage of the nanowire assembly is the impurity-vortex line interaction, which has been modelled by OT-DFT calculations. 43

The actual impurity trapping event is clearly a dynamic process, see Sec. VO. Assuming that the impurity impact velocity remains small and the vortex line geometry does not deviate from linear geometry, static interaction energy potentials for the vortex-impurity 


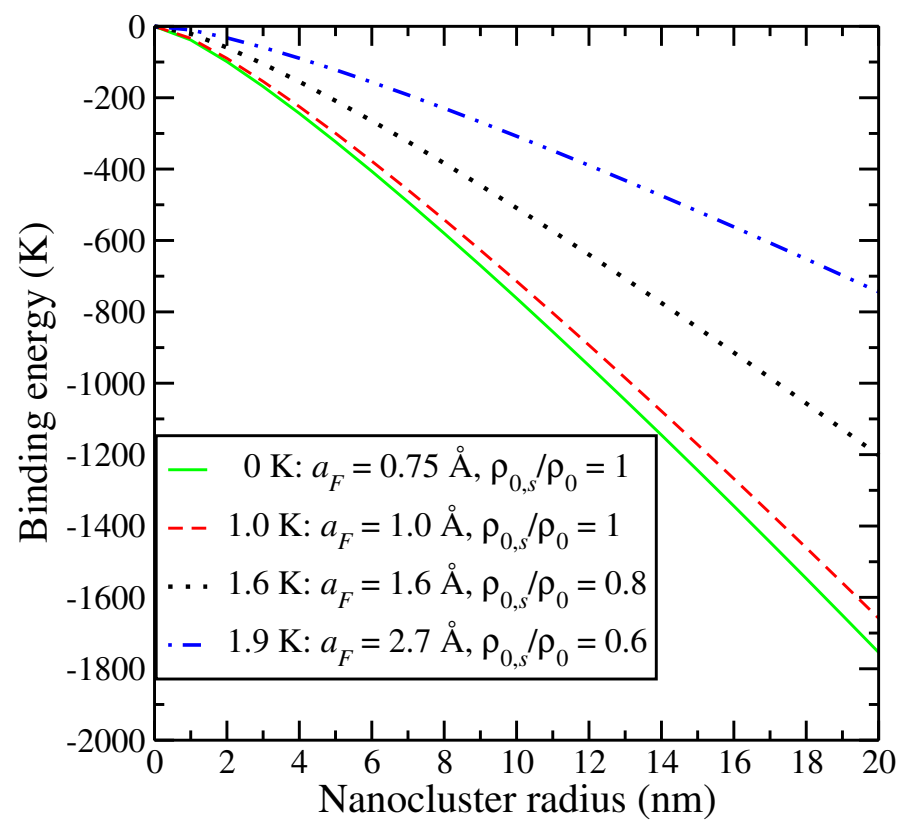

FIG. 31. Nanocluster-vortex binding energies according to Eq. 75 as a function of the cluster size at indicated temperatures. $\stackrel{43}{\underline{4}}$

interaction can be obtained. This calculation can be carried out in imaginary-time where the vortex line structure is imposed by the initial guess given by Eq. (28). The position of the impurity must remain fixed during the calculation, which can be imposed by including the following penalty term in the external potential 43 (see also Eq. (24))

$$
V[\Psi, \mathbf{r}]=2 \lambda_{C}\left(z-z_{0}\right) \int d \mathbf{r}^{\prime} \Psi^{*}\left(\mathbf{r}^{\prime}\right)\left(z^{\prime}-z_{0}\right) \Psi\left(\mathbf{r}^{\prime}\right)
$$

where $\lambda_{C} \sim 10^{-5}$ a.u., and the impurity is constrained along the $z$-axis (perpendicular to the vortex line) at position $z_{0}$. Calculation of the total energy of the system as a function of the distance between the vortex line and the impurity yields the static interaction potential.

In addition to providing the total binding energies of impurities to vortex lines, the interaction energies can be rationalised by the Donnelly-Parks potential function ${ }^{245}$ (see also Eq. 29)

$$
V_{\text {im-vortex }}(r)=-2 \pi \rho_{0, s}\left(\frac{\hbar^{2}}{m_{4}}\right)^{2} \int_{0}^{R} d \xi \frac{\left(R^{2}-\xi^{2}\right)^{1 / 2} \xi}{\left[\left(\xi^{2}+r^{2}+a_{F}^{2}\right)^{2}-4 r^{2} \xi^{2}\right]^{1 / 2}}
$$


where $R$ represents the classical radius for the spherical cavity containing the impurity, $r$ is the vortex core-impurity distance, $\rho_{0, s}$ is the bulk superfluid helium density, and $a_{F}$ is the effective healing length. Non-linear least squares fit of this expression to the interaction potential energy curves from OT-DFT provides effective size estimates for both $R$ and $a_{F}$. Some of the obtained results are shown in Table III.

Nanowires in liquid helium are believed to form through recombination of metal nanopar-

ticles in the vortex rather than through building up from individual atoms. $\frac{238 / 246}{2}$ The size of such particles is too large for any practical OT-DFT calculation. Therefore, the binding energy estimates for nanoparticles bound to rectilinear vortex lines can only be obtained from Eq. (75). Note that this assumes that the vortex lines are linear and longer than the diameter of the approaching nanoparticle. The binding energy data as a function of the nanoparticle radius is shown in Fig. 31. The effect of temperature was included in the estimate by varying the superfluid density $\left(\rho_{0, s}\right)$ and the healing length $\left(a_{F}\right)$ accordingly. While the binding energies quickly exceed $1000 \mathrm{~K}$, under real experimental conditions this may rather be limited by the dimensions of the vortex line itself.

\section{Vortex arrays in ${ }^{4} \mathrm{He}$ droplets}

Together with the frictionless motion of impurities at velocities below the Landau critical velocity in superfluid ${ }^{4} \mathrm{He}$, the appearance of quantised vortices is another signature of superfluidity. Helium remains at rest when its container is rotated, until a critical angular velocity is reached. This leads to the appearance of vortices with quantised velocity circulation in units of $h / m_{4}$, where $h$ is the Planck constant.

The vortex line distributions in superfluid ${ }^{4} \mathrm{He}$ were first imaged by Williams and Packard ${ }^{247}$ by means of light scattered by electrons attached to the vortex lines; quantised vortices have also been visualised by suspending micron-sized solid particles of hydrogen in bulk superfluid ${ }^{4} \mathrm{He} \cdot{ }^{248 \mid 249}$ More recently, femtosecond single-shot x-ray diffraction imaging of Xe doped ${ }^{4} \mathrm{He}$ droplets employing a free electron laser, revealed Bragg spots confirming the existence of quantum vortex arrays in helium droplets. 29|116/117 This result shows that large ${ }^{4} \mathrm{He}$ droplets containing about $10^{10}$ atoms are superfluid.

As discussed earlier, DFT has proven to be a very useful theoretical tool to study vortices in liquid ${ }^{4} \mathrm{He}$. In most recent applications, vortex arrays in a rotating ${ }^{4} \mathrm{He}$ nanocylinder ${ }^{73}$ 


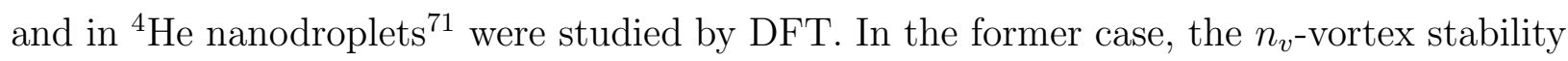
diagram was computed and compared with that of classical vortex lines in an inviscid, incompressible fluid. Vortex array configurations in a rotating cylinder - as that shown in Fig. 32 - can be completely characterised within the Onsager-Feynman model by the dimensionless energy per unit length $\mathcal{E} \equiv\left(m_{4} / \rho_{0} \pi \hbar^{2}\right) E$, the dimensionless angular velocity $\Omega \equiv R^{2} m_{4} \omega / \hbar$, and the scaled radial positions of the vortices $r_{i} / R$. Here $\rho_{0}=0.0218 \AA^{-3}$ is the bulk density and $R$ is the radius of the cylinder. 250 By scaling the calculated values to millimeter-scale, the nanoscale results agree with the experimental data on vortex arrays observed in the bulk liquid. $\underline{247}$

The appearance of vortex arrays in rotating ${ }^{4} \mathrm{He}$ nanodroplets at $T=0$ was recently investigated by DFT. ${ }^{71}$ The results were compared with the theory developed for rotating classical fluid spheres, which was earlier used to analyze the shape and vorticity in helium droplet experiments. ${ }^{29}$ In agreement with the experimental data, the droplets remain stable well above the stability limit predicted by classical theories despite their large shape deformations due to rotation. $\stackrel{251 \mid 252}{2}^{2}$ Vorticity inside the droplets changes their appearance from ellipsoidal to oblate and 'wheel'-shaped with small and large vortex densities, respectively. In agreement with the experiments, the latter shape exhibits nearly flat upper and lower surfaces. Selected vortex array configurations for a $N_{4}=15000$ droplet are shown in Fig. 33 .

The above results can be compared with the experimental data obtained for much larger droplets once they are scaled by a dimensionless characteristic rotational velocity $\Omega$

$$
\Omega=\sqrt{\frac{m_{4} \rho_{0} R^{3}}{8 \gamma} \omega}
$$

where $\gamma=0.274 \mathrm{~K}^{-2}$ is the surface tension of the liquid. For a $N_{4}=15000$ droplet, $\Omega=1$ corresponds to $\omega=1.13 \times 10^{10} \mathrm{~s}^{-1}$.

The data shown in Fig. 33 demonstrate that the rotating droplet aspect ratio - defined as $b / a$ where $a$ is the short half-axis and $b$ the long half-axis length - depends on the angular frequency. The calculated $b / a$ vs. $\Omega$ is plotted in Fig. 34 together with the classical model prediction, which was used in Ref. 29 to interpret the experimental observations. Notice the gaps that appear in the calculated data, which reflect the presence of forbidden values of the angular momentum per atom. Similar gaps have also been observed in calculations modelling trapped rotating BEC. ${ }^{253}$ Despite the apparent differences between rotating classical and 


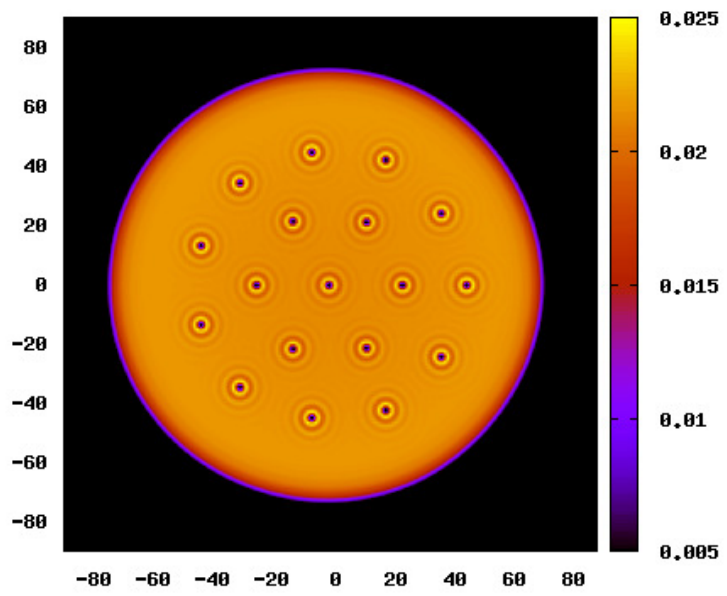

FIG. 32. Lowest energy stationary 18-vortex configuration in a nanocylinder of $R=71.4 \AA$ radius at $\Omega=29.6$ (defined in Eq. $(76)$ ). Distances are specified in $\AA$. The contour colors correspond to density values between $\rho=0$ and $\rho=0.03 \AA^{-3} \underline{\text { ?73 }}$

superfluid droplets, the relationship between the aspect ratio and the angular frequency looks very similar. The classical model underestimates the angular frequency only by less than $10 \%$ for large vortex arrays.

The experimental diffraction images of Xe doped He droplets ( $200 \mathrm{~nm}$ diameter $)$ have revealed configurations made of symmetrically arranged vortex arrays that are decorated with Xe clusters at unexpectedly large distances from the centre of the droplet!116 These observations have been explained in terms of angular momentum conservation. When the Xe atoms are drawn to the vortex cores, they start rotating along with the vortex array. The increased moment of inertia due to the additional Xe atoms must decrease the rotational angular velocity of the vortex array causing it to expand such that the inter-vortex distances increase. Note that the DFT results discussed above refer to pure helium droplets. Results for vortex arrays in helium nanocylinders that are decorated with Xe atoms are discussed in Ref. 73 . 

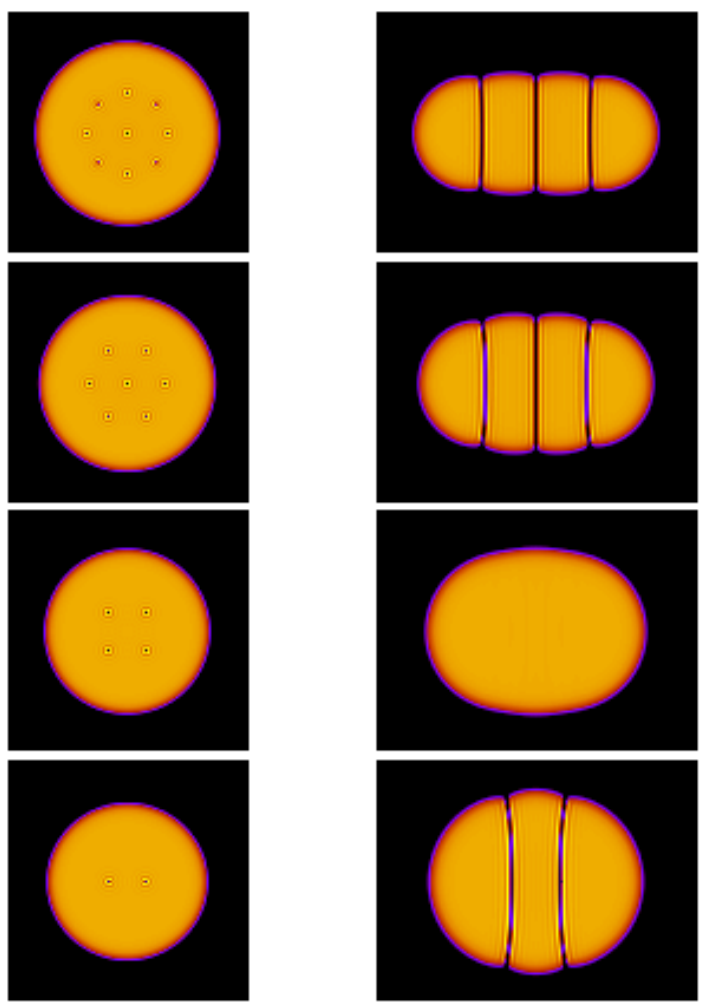

FIG. 33. From bottom to top, helium droplet configurations hosting $n_{v}=2,4,7$ and 9 vortex lines. The left column shows the helium density on the $z=0$ symmetry plane (top view) and the right column on the $x=0$ plane (side view)! $[1]$

The above system was recently modelled by DFT using Ar doped helium droplets because the calculations are technically simpler than for Xe. ${ }^{231}$ The system considered consisted of an array of six vortex lines filled with $\mathrm{Ar}$ atoms inside a $N_{4}=15000$ droplet ( $\sim 11 \mathrm{~nm}$ diameter). In qualitative agreement with the experimental observations, the calculations show that the doping of the vortex cores substantially increases the rigidity of the system. This, in turn, makes the droplet stable at lower angular velocities and increases the inter-vortex distances. In contrast, a pure helium droplet with the same vortex array would have been unstable and the vortices would have been expelled off the droplet. Moreover, the solvation potential effect - which tends to attract the Ar atoms towards the center of the droplet - becomes apparent since, below some critical value of the angular velocity, the vortices cease to displace towards 


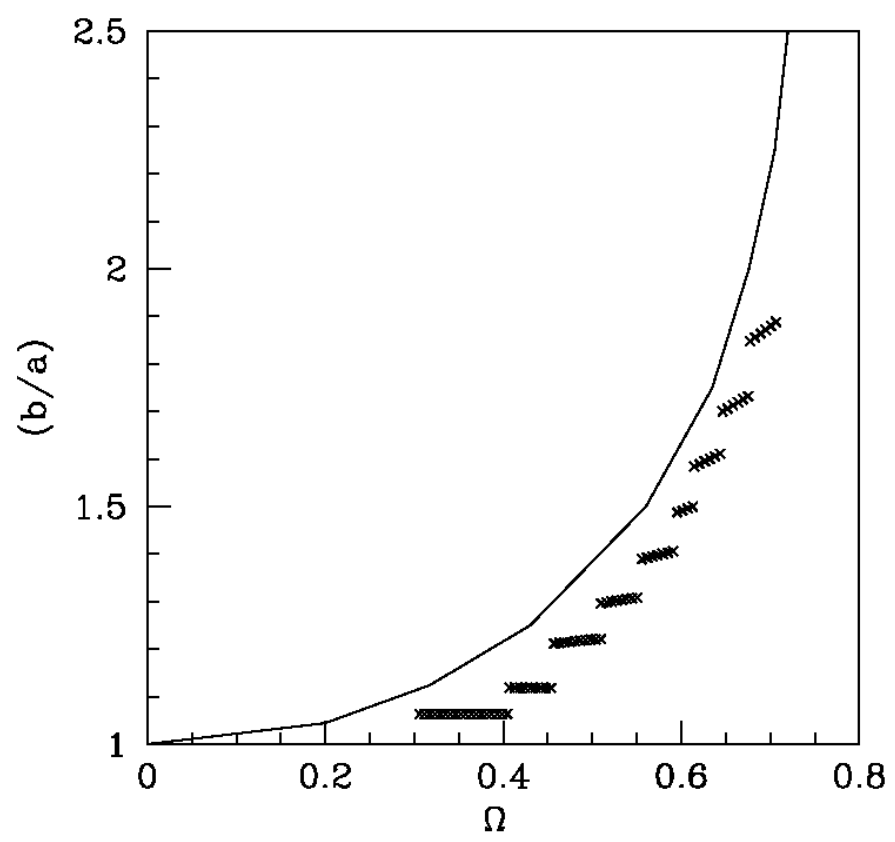

FIG. 34. Aspect ratio $b / a$ as a function of the dimensionless angular velocity $\Omega$ [defined in Eq. (76)] for a $N_{4}=15000$ droplet. The solid line shows the experimental curve obtained by using the classical model. Discontinuities in $b / a$ vs. $\Omega$ appear at the values corresponding to phase transitions between configurations with different number of vortices (from 1 to 9 ) 71

the surface and the system reaches an equilibrium maximum distance of the vortices from the droplet centre.

\section{N. Dynamics of alkali atoms excited on the surface of ${ }^{4} \mathrm{He}$ droplets}

In a quest to understand how chemical reactions proceed in ultra-cold helium droplets, real time dynamics of photoexcited and photoionised atoms and molecules have been studied extensively by TDDFT. These processes share some elements with condensed phase chemical reactions, namely the dynamic liquid rearrangement and strong coupling of the electronic degrees of freedom to the surrounding liquid.

Photodissociation of $\mathrm{Cl}_{2}$ and the following relaxation dynamics in ${ }^{4} \mathrm{He}$ droplets has been recently addressed $\sqrt[254]{256}$ These studies constituted the first application of TDDFT to de- 
scribe photodissociation of a homonuclear diatomic molecule embedded in superfluid helium. Related processes experimentally studied include photodissociation of alkyl iodides ${ }^{257}-259$ and $\mathrm{Cr}_{2}$ molecules ${ }^{[260}$ From the theory point of view, a major technical problem in modelling such systems is that a large amount of energy is deposited into the liquid. Unless the number of atoms in the droplet is very large (millions of atoms), the droplet is expected to disintegrate on a sub-picosecond time scale. Since large helium droplets can be approximated by the bulk liquid, theoretical calculations could be carried out in the bulk to avoid helium evaporation from the droplet surface. Irrespective of the geometry of the helium sample, these calculations require accurate dimer/molecule-helium interaction potentials, which poseses a challenge even to modern electronic structure methods.

The dynamics following photoexcitation of alkali atoms attached to helium droplets has been investigated in a series of joint experimental and theoretical works. ${ }^{7600}$ Photoelectron spectroscopy revealed that, upon excitation from the ground to the first excited s-state, alkali atoms desorb from the droplet surface. The mean kinetic energy of these atoms, which can be detected by ion imaging, shows a linear dependence on the excitation energy. TDDFT calculations on these systems revealed that the desorption process is accompanied by the creation of highly non-linear liquid density waves in the droplet that propagate at supersonic velocities.

The test-particle method described in Sec. IVB was introduced in the context of optical excitation of $\mathrm{Li}$ and $\mathrm{Na}$ atoms - sitting on the surface of helium droplets - to their first excited s-state ${ }^{80}$ The ejected alkali metal atoms acquire high velocities, which makes the direct numerical solution of the Schrödinger equation difficult in the TTDFT context. An overview of the time evolution of the $\mathrm{Na}^{4}{ }^{4} \mathrm{He}_{1000}$ complex following the $4 \mathrm{~s} \leftarrow 3$ s excitation is shown in Fig. $35{ }^{80}$ The sudden repulsive interaction between the excited $\mathrm{Na}$ atom and the droplet creates a series of supersonic shock waves in the droplet, which indicates that a significant fraction of the energy introduced by the optical excitation is transferred directly into the droplet. These waves were observed to travel at velocities ranging from 370 to 890 $\mathrm{m} / \mathrm{s}$, which are of similar magnitude as recently observed in laser ablation experiments in the bulk liquid ${ }^{261}$ Furthermore, the moving high density peak, which originates from the first solvation shell, exhibits (bright) solitonic features such as constant propagation velocity (ca. $590 \mathrm{~m} / \mathrm{s}$ ) and no spatial dispersion. A similar result was found for the ejection of $\mathrm{Rb}$ and Cs atoms from helium droplets when excited from $5 \mathrm{~s}$ to $6 \mathrm{~s}$ and from $6 \mathrm{~s}$ to $7 \mathrm{~s}$ states, 

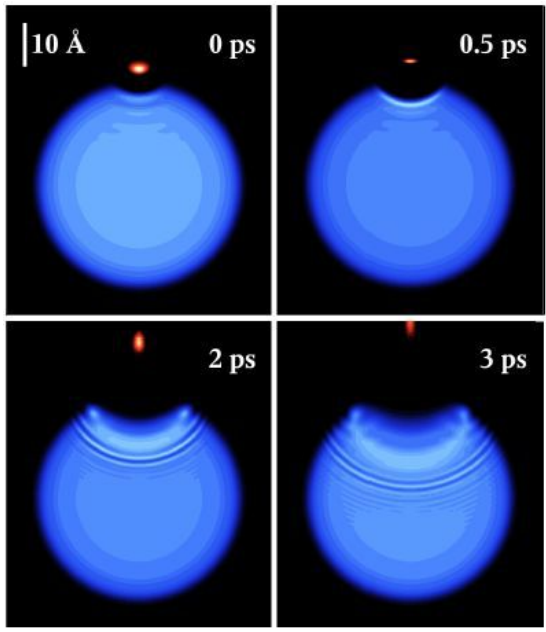
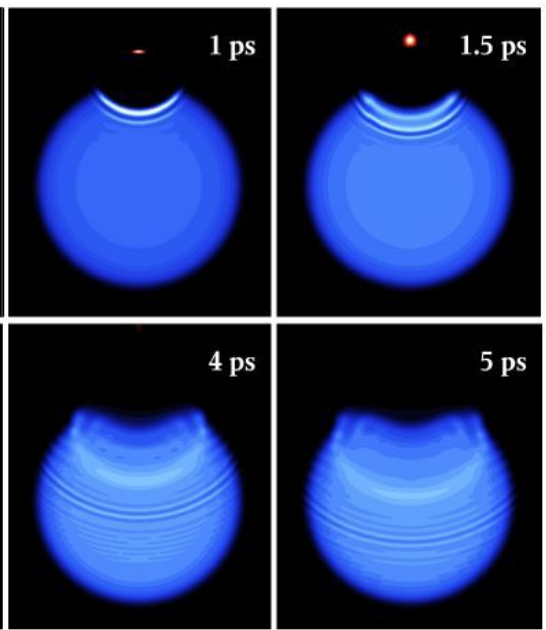

FIG. 35. Time evolution of the $\mathrm{Na} @{ }^{4} \mathrm{He}_{1000}$ complex upon $4 \mathrm{~s} \leftarrow 3$ s excitation. 80

respectively!

Detailed information about the kinematics of the process can be obtained by monitoring the kinetic energy of the atoms desorbed from the droplets as a function of the excitation energy. ${ }^{76 / 80}$ The experimental results for $\mathrm{Cs}$ and $\mathrm{Rb} \mathrm{b}^{76}$ are shown in Fig. 36. The calculated points in that figure were obtained by starting TDDFT simulations from various impurity positions determined by a constrained minimization of the total energy of the complex. Whereas the light alkali metal atoms ( $\mathrm{Li}$ and $\mathrm{Na}$ ) require the use of the test-particle method, heavy alkali metals ( $\mathrm{Rb}$ and $\mathrm{Cs}$ ) can be treated classically.

For all alkali metals, the kinetic energy of the ejected atom exhibits a linear dependence on the excess excitation energy of the $(n+1) \mathrm{s} \leftarrow n$ s transition. This indicates that, despite its apparent complexity, the ejection process can be well represented by a "pseudo-diatomic model ${ }^{262}$ in which the droplet is represented by one big atom bound to the alkali. By imposing energy and linear momentum conservation during the instantaneous ejection of the alkali atom from the droplet, the relative kinetic energy can be written as

$$
E_{k i n}=\eta\left(\hbar \omega-\hbar \omega_{0}\right)
$$

where $\omega$ denotes the excitation and $\omega_{0}$ the atomic transition frequencies. The slope $\eta$ is related to the effective mass of the helium droplet (the mass of the helium atoms effectively 


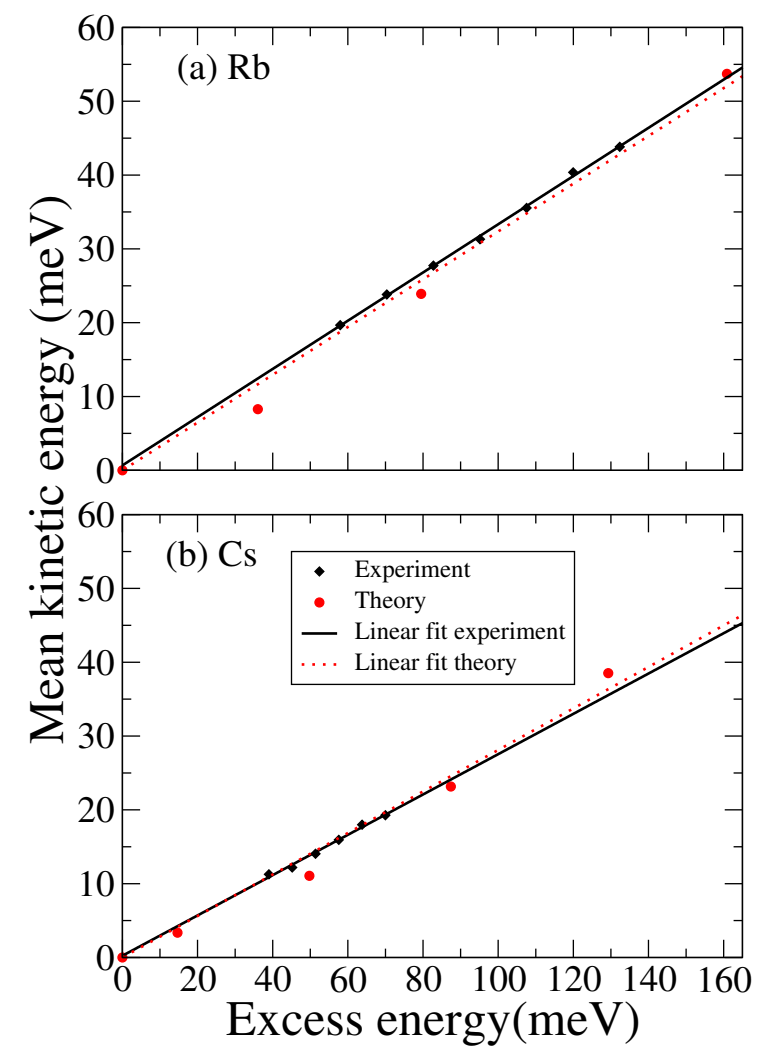

FIG. 36. Mean kinetic energies of Rb (a) and Cs (b) atoms desorbing from helium droplets upon $6 \mathrm{~s} \Sigma$ and $7 \mathrm{~s} \Sigma$ state excitation, respectively. Straight and dotted lines: linear fits to the experimental and theoretical data, respectively ${ }^{76}$

participating in the interaction with the alkali atom), $m_{\mathrm{eff}}$, by

$$
\eta=\frac{m_{\mathrm{eff}}}{m_{\mathrm{eff}}+m_{\mathrm{Ak}}} \Longrightarrow m_{\mathrm{eff}}=\frac{\eta}{1-\eta} m_{\mathrm{Ak}}
$$

Fitting the experimental and simulation data to Eq. (77) yields the results summarised in Table IV. It can be seen that $m_{\text {eff }}$ increases with the mass of the alkali atom as indicated by Eq. (78). The variation of the corresponding number of helium atoms reflect the differences in the dimple structure and the excited state interaction with the droplet. ${ }^{76}$ Note the lack of data for the $5 \mathrm{~s} \leftarrow 4$ s transition for the $\mathrm{K}$ atom. While there is no difficulty in simulating 


\begin{tabular}{|c|c|c|c|c|c|}
\hline \multicolumn{2}{|c|}{$\mathrm{Ak} \mathrm{m}_{A k}(\exp )$} & $\eta(\exp )$ & $\eta(\mathrm{th})$ & eff (ex & $\mathrm{A}_{\text {eff }}(\mathrm{th})$ \\
\hline & {$[\mathrm{amu}]$} & & & {$[\mathrm{amu}]$} & {$[\mathrm{amu}]$} \\
\hline $\mathrm{Li}$ & 6.94 & 0.687 & 0.756 & 15.2 & 21.5 \\
\hline $\mathrm{Na}$ & 23.0 & 0.516 & 0.583 & 24.6 & 32.2 \\
\hline $\mathrm{Rb}$ & 85.5 & 0.327 & 0.324 & 41.9 & 41.0 \\
\hline Cs & 132.9 & 0.281 & 0.273 & 51.8 & 50.5 \\
\hline
\end{tabular}

TABLE IV. Some characteristics of the experimental and theoretical kinetic energy distributions of the desorbed alkali atoms, $\frac{76[80}{,}$ see text for details.

it with TDDFT, this transition may overlap with $3 \mathrm{~d} \leftarrow 4$ s and this complicates the analysis of the experimental results.

In addition to the $(n+1) \mathrm{s} \leftarrow n$ s transitions discussed above (see also Refs. 106, 107, 202, and 263), the lower energy $n \mathrm{p} \leftarrow n$ s transitions have also been addressed in a series of experimental and theoretical studies. $\frac{85|97| 102[103 / 264] 266}{26}$ The first expectation was that alkali metal atoms would always detach from the droplets upon $n \mathrm{p} \leftarrow n$ s excitation. However, only the light alkali metals such as $\mathrm{Li}, \mathrm{Na}$, and $\mathrm{K}$ appear to detach; the heavier alkalis ( $\mathrm{Rb}$ and Cs) may remain attached if they are excited with energies close to that of the D1 line in the gas-phase. $204 \mid 265$

Photoexcitation and photoionisation of $\mathrm{Rb}$ atoms attached to helium droplets has been studied in real-time dynamics experiments. ${ }^{267}$ It was shown that excitation of Rb atoms from the $5 \mathrm{~s}$ to the $6 \mathrm{p}$ states leads to their detachment. Upon subsequent ionisation of the excited $\mathrm{Rb}$ atom $\left(\mathrm{Rb}^{*}\right)$, the interaction with helium becomes attractive. Hence, depending on the time delay $\tau_{D}$ between the excitation and ionisation laser pulses, the resulting ion may be ejected as a bare $\mathrm{Rb}^{+}$cation or as a $\mathrm{Rb}^{+} \mathrm{He}_{n}$ complex, or it can be drawn into the droplet. The critical time $\tau_{c}$ separating these processes is called the fall-back time.267

Recently, this study has been extended to the Rb $5 \mathrm{p} \leftarrow 5$ s transition. 31 Both transitions have been simulated with TDDFT as described in Sec. IVA. The Rb atom located on the droplet surface is first photoexcited to either the $5 \mathrm{p}$ or the $6 \mathrm{p}$ state $\left({ }^{2} \Sigma_{1 / 2},{ }^{2} \Pi_{1 / 2}\right.$ or $\left.{ }^{2} \Pi_{1 / 2}\right)$ and the system evolves on the excited state potential energy surface. After a fixed delay, $\mathrm{Rb}^{*}$ is photoionised, which is simulated by suddenly switching the interaction potential to $\mathrm{Rb}^{+}-\mathrm{He}$. It was found that the desorption process for the $6 \mathrm{p}$ state is impulsive whereas the 


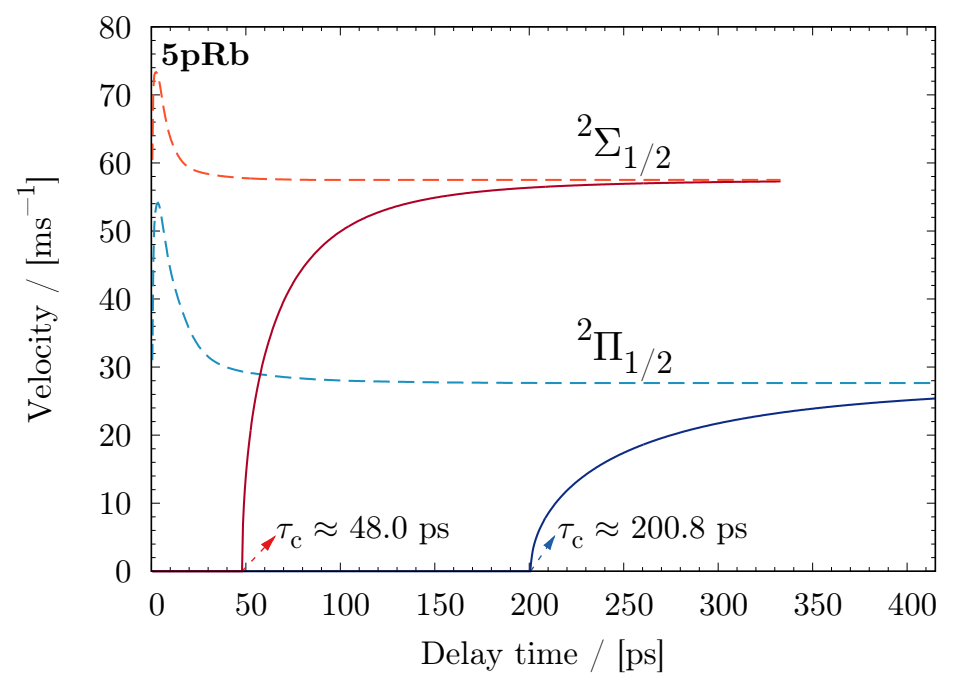

FIG. 37. Velocity of the $\mathrm{Rb}^{*}$ atom in the $5 \mathrm{p}^{2} \Sigma_{1 / 2}$ and $5 \mathrm{p}^{2} \Pi_{1 / 2}$ states (dashed lines) and the $\mathrm{Rb}^{+}$cation produced by photoionisation (solid lines) after a given delay time vs. delay time. The fall-back times $\tau_{c}$ are indicated by arrows.

behavior of the $5 \mathrm{p}$ state is intermediate between impulsive and complex dissociation. The desorption time scales are also very different for the two states: $\sim 1$ ps for $6 \mathrm{p}$ and $\sim 100 \mathrm{ps}$ for $5 \mathrm{p}$.

The velocities of $\mathrm{Rb}^{*}$ and the $\mathrm{Rb}^{+}$as a function of the delay time are shown in Fig. 37 for the $5 \mathrm{p} \leftarrow 5 \mathrm{~s}$ transition. Note that the largest (asymptotic) velocity of $\mathrm{Rb}^{+}$is simply given by the corresponding asymptotic velocity of $\mathrm{Rb}^{*}$ because the ion escape velocity is not affected by the droplet at large distances. TDDFT simulations have also been extended to the desorption of $\mathrm{Cs}$ following the $6 \mathrm{p} \leftarrow 6$ s excitation. 268 The general features of the dynamics appear very similar to $\mathrm{Rb} 5 \mathrm{p} \leftarrow 5 \mathrm{~s}$.

The TDDFT simulations for Rb and Cs atoms excited from their $n$ s ground to their $n \mathrm{p}$ excited state can be summarised as follows: i) excitation to the ${ }^{2} \Sigma_{1 / 2}$ or ${ }^{2} \Pi_{1 / 2}$ state leads to desorption of the excited alkali atom $\left(\mathrm{Ak}^{*}\right)$; ii) excitation to the ${ }^{2} \Pi_{3 / 2}$ state produces an exciplex within $\sim 10 \mathrm{ps}$, which remains attached to the droplet surface.

Experiments and TDDFT calculations agree on the ejection of $\mathrm{Ak}^{*}{ }^{2} \Sigma_{1 / 2}$ state from helium droplets. At first sight, the results for ${ }^{2} \Pi_{3 / 2}$ and ${ }^{2} \Pi_{1 / 2}$ states seem to disagree with 


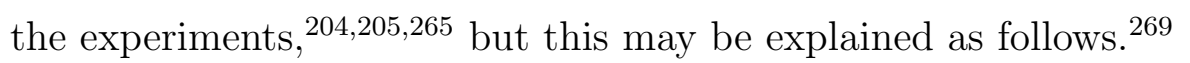

- In the case of the ${ }^{2} \Pi_{1 / 2}$ state, the experiments have explored the low energy region of the D1 line whereas the TDDFT dynamics was initiated using a configuration that corresponds to the D1 resonance. Hence, the initial energy in the TDDFT simulation is larger than in experiments.

- In the case of the ${ }^{2} \Pi_{3 / 2}$ state, it is worth stressing that the electronic state of the ejected $\mathrm{Ak}^{*}$ was not determined in the experiments. Only the state to which the Ak atom was excited is determined (by the excitation laser wavelength). In the case of $\mathrm{Rb}^{*}$, experimental indications point to a non-radiative relaxation of the $5 \mathrm{p}^{2} \Pi_{3 / 2}$ to the $5 \mathrm{p}^{2} \Pi_{1 / 2}$ state. $\frac{31}{\text { If }}$ this happens, the relaxed state may be a $\mathrm{RbHe}^{2} \Pi_{1 / 2}$ exciplex. The energy available from this relaxation process is about $190 \mathrm{~cm}^{-1}$. If even only one third of this energy is given to the $\mathrm{RbHe}^{2} \Pi_{1 / 2}$ exciplex as additional initial kinetic energy, it is ejected from the droplet according to the TDDFT simulations. This process is compatible with the possibility of forming exciplexes in the ${ }^{2} \Pi_{3 / 2}$ state which could remain attached to the droplet ${ }^{270}$ if no electronic relaxation occurs. Note that no exciplex was produced upon direct ${ }^{2} \Pi_{1 / 2}$ excitation because of a barrier preventing its formation. A similar relaxation process is also fully compatible with the experimental observations for $\mathrm{Ag} \underline{44}$

In a related study, DMC calculations have been carried out for $\mathrm{Rb}^{*}$ in a small cluster or on a helium film, which can be considered as a simplified model of a large droplet surface. ${ }^{266}$ This study found that $\mathrm{Rb}^{*}$ stabilizes as a weakly bound metastable $\mathrm{Rb}^{2} \Pi_{1 / 2}$ (not an exciplex) that forms a shallow dimple structure on the surface. There is no contradiction between the DMC and the dynamic TDDFT calculations in this respect: The DIM potentials indeed display a shallow minimum for this state so that the imaginary-time DFT relaxation would also yield a weakly bound $\mathrm{Rb}^{*}$. However, the energy available in the real time dynamics hinders the formation of this relaxed, weakly bound state. The same group later investigated Rb excited to the ${ }^{2} \Pi_{3 / 2}$ state, ${ }^{271}$ showing the appearance of a linear exciplex to which more helium atoms are attached, preferentially on one end of the linear exciplex. The TDDFT simulations are in agreement with these findings that, to a large extent, can be understood by inspecting the $V_{\lambda}$ DIM potentials, Eq. (44), as plotted in Fig. 38. Notice for example the appearance of two deep wells in the ${ }^{2} \Pi_{3 / 2}$ potential; the filling of these wells by helium atoms yields the $\mathrm{Rb}_{2}{ }^{2} \Pi_{3 / 2}$ linear exciplex. 

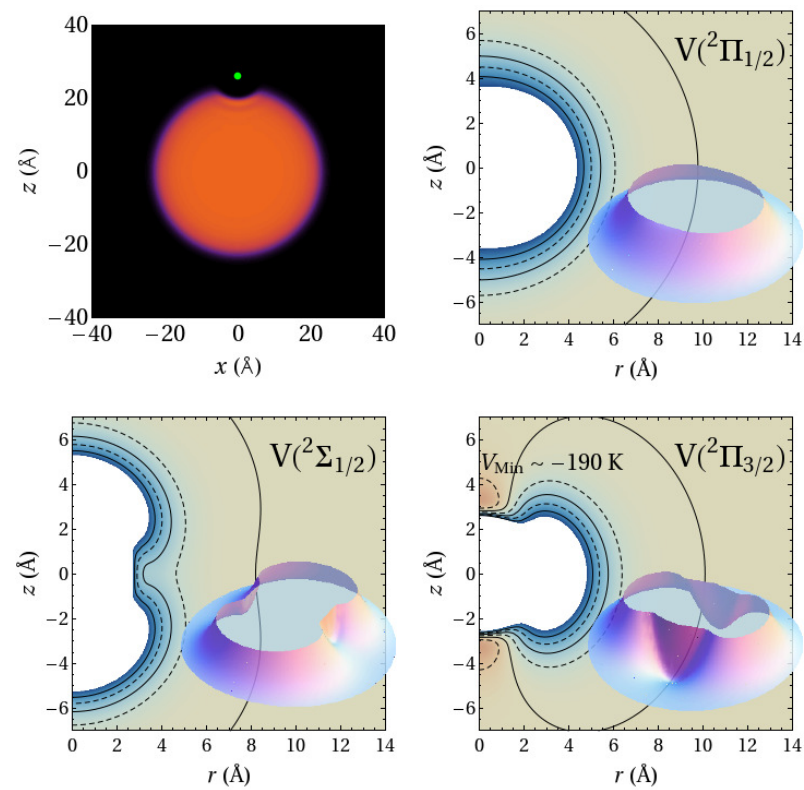

FIG. 38. Top left panel: Equilibrium dimple configuration of Rb taken as starting point for the dynamics; the $\mathrm{Rb}$ atom is at $26.3 \AA$ from the $\mathrm{COM}$ of the droplet. The other panels display the ${ }^{2} \mathrm{P} V_{\lambda} 5 \mathrm{p}$ Rb-He potentials (Eq. (44)) corresponding to this configuration. Regions where the potentials are attractive (repulsive) are represented in brown (blue). The outermost equidensity line corresponds to zero potential. The $5 \mathrm{p}^{2} \Sigma_{1 / 2}$ and $5 \mathrm{p}^{2} \Pi_{1 / 2}$ potentials have a shallow attractive minimum of about $1 \mathrm{~K}$ depth at a distance of $\sim 10 \AA$.

\section{O. Capture of impurities by ${ }^{4} \mathrm{He}$ droplets}

The previous sections frequently consider situations that appear after an impurity has been captured by a helium droplet or after it has been injected into liquid helium. It is well known that helium droplets are able to capture atoms and molecules, as first shown for Ne atoms. $\frac{273}{7}$ This finding has had a tremendous impact on low temperature experiments as the technique allows to use helium droplets as an ultra-cold matrix.

The pickup process of gas phase Ar, $\mathrm{Kr}$, and Xe atoms by ${ }^{4} \mathrm{He}_{N} \operatorname{droplets}\left(N_{4}>10^{3}\right.$ atoms $)$ produced in nozzle-beam expansions was first studied by Toennies and co-workers! 126 These experiments characterised the deflection of a helium droplet beam by a secondary crossed beam made of rare gas atoms. This cross-beam technique was later used to characterize the 


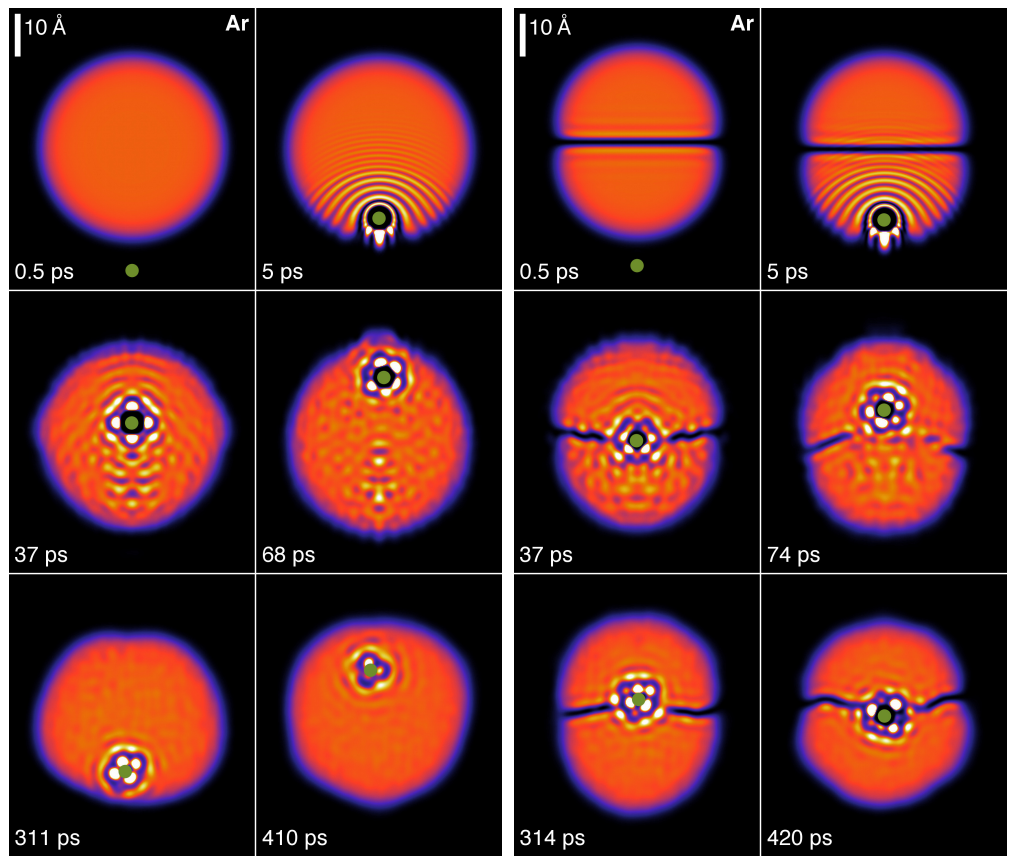

FIG. 39. (a) Simulation of an Ar atom (green dot) approaching a ${ }^{4} \mathrm{He}_{1000}$ droplet from below at $v_{0}=360 \mathrm{~m} / \mathrm{s}$. The corresponding time is indicated in each frame. (b) Same as (a) but the droplet hosts a vortex line. We have included mp4 movies as Supplemental Material (on line) that show the complete simulations corresponding to this figure.

helium droplet density in the beam by comparing the measured integral cross-section with the helium droplet density profiles predicted by DFT calculations. 274|275

Theoretical work addressing the capture process is scarce. The earliest work on the scattering of ${ }^{4} \mathrm{He}$ atoms by ${ }^{4} \mathrm{He}$ droplets was largely inspired by the nuclear physics optical model $\left[\frac{276}{2}\right.$ More recently, scattering of helium atoms from inhomogeneous quantum liquids has also been considered. 277278

\section{Pure droplets}

The first TDDFT calculations modelling the capture of impurities by helium droplets were recently carried out for heliophobic $(\mathrm{Cs})^{279}$ and several heliophilic atoms (Ne, Xe and Ar) $\stackrel{231 / 2801281}{ }$ The heavy impurities were treated classically whereas the lighter Ne was treated 


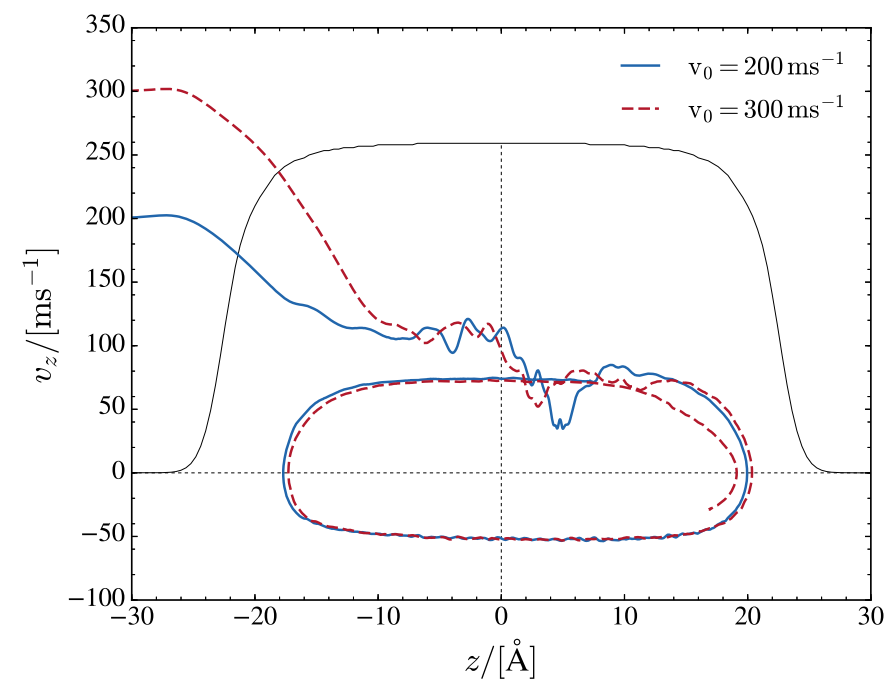

FIG. 40. Phase space evolution of the Xe atom for two values of the initial velocity $v_{0}$ (200 and $300 \mathrm{~m} / \mathrm{s}$ ) during a head-on collision with a ${ }^{4} \mathrm{He}_{1000}$ droplet. The initial droplet density profile is also shown in arbitrary density scale.

quantum mechanically; however, the collision process considered was strictly 1D (see Sec. IV A.

Depending on the energy and the impact parameter of the impinging atom, a rich variety of dynamical phenomena may be observed. 231279 DFT calculations have shown that for a Cs atom to be trapped on the droplet surface, its excess kinetic energy must be transferred to the droplet very efficiently because the Cs-droplet binding energy is only $10.5 \mathrm{~K}$. In a head-on collision with a heliophilic Xe atom, whose binding energy is $316.3 \mathrm{~K}$, if the impact velocity is sufficiently high $\left(v_{0}>600 \mathrm{~m} / \mathrm{s}\right)$, the Xe atom may pass through the droplet; 281 otherwise it remains trapped inside. As an example, Fig. 39 shows the head-on collision of an Ar atom at $v_{0}=360 \mathrm{~m} / \mathrm{s}$ with a ${ }^{4} \mathrm{He}_{1000}$ droplet.

Most of the excess kinetic energy of the impurity is deposited into the droplet, which results in the ejection of He atoms and the emission of sound and shock waves. Contrary to the naive expectation that the average energy per ejected He atom simply corresponds to its binding energy $(\sim 7 \mathrm{~K})$, the atoms ejected at early times (prompt-emitted atoms) carry significant amounts of kinetic energy - see Table V. Whether the impurity is heliophilic or 

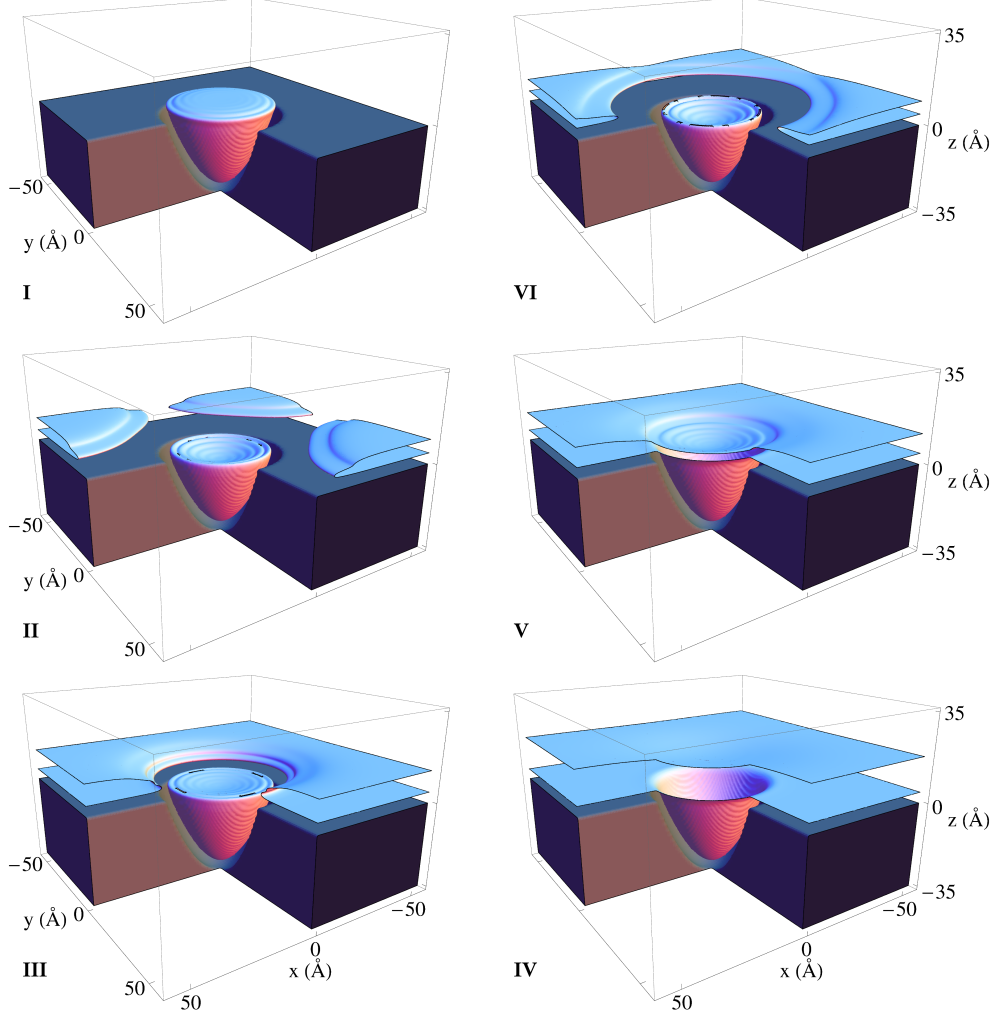

FIG. 41. Liquid ${ }^{4} \mathrm{He}$ on a Na patterned surface. ${ }^{272}$ The panels show some illustrative equilibrium configurations for different coverages; the panels display the isodensity surfaces drawn at a value which is half the bulk liquid density at $T=0\left(\rho_{0}=0.0218 \AA^{-3}\right)$. The dark area represents the Na planar surface.

heliophobic plays a role in the process. For example, for heliophilic Xe, 18 He atoms are ejected after 200 ps for $v_{0}=200 \mathrm{~m} / \mathrm{s}$ whereas only 6 He atoms are ejected in the case of heliophobic Cs during the same period of time.279

For an impurity to be captured by a helium droplet, the excess kinetic energy of the impurity must be dissipated such that it becomes less than the impurity-droplet binding energy. Fig. 40 shows the trajectory in phase space of a Xe atom captured in a ${ }^{4} \mathrm{He}_{1000}$ droplet for a head-on collision at $v_{0}=200$ and $300 \mathrm{~m} / \mathrm{s}^{231}$ It can be seen that for these collisions - corresponding to thermal velocities - the motion of the impurity inside the droplet is independent of $v_{0}$ to some extent. We attribute this to the fact that dissipation 


\begin{tabular}{cccc}
\hline \hline Species & $v_{0}(\mathrm{~m} / \mathrm{s})$ & $N_{e}$ & $E_{e}(\mathrm{~K})$ \\
\hline $\mathrm{Xe}$ & 200 & 18 & 19 \\
& 300 & 28 & 23 \\
& 400 & 37 & 30 \\
\hline $\mathrm{Ar}$ & 360 & 16 & 22 \\
\hline \hline
\end{tabular}

TABLE V. Number of ejected helium atoms $\left(N_{e}\right)$ and average energy per ejected atom $\left(E_{e}\right)$ for the indicated head-on collisions during the first 200 ps. $\stackrel{231}{ }$

occurs mostly during the very first stages of the process. $231 \mid 281$

In grazing collisions not only excess energy but also angular momentum is deposited into the droplet. This allows to visualize the resulting irrotational superfluid flow inside the droplet and to calculate the capture cross-section. At low energies and small impact parameters, the impurity is captured by the droplet and may even orbit around the droplet COM.

A simple expression for the capture cross-section of classical dopants can be obtained provided that the reduced de Broglie wavelength of the impurity is much smaller than the droplet ${ }^{279}$

$$
\sigma(E)=\frac{\pi}{\kappa^{2}} \sum_{\ell=0}^{\ell_{c r}}(2 \ell+1)=\frac{\pi}{\kappa^{2}}\left(\ell_{c r}+1\right)^{2}
$$

where $E$ is the energy available in the COM frame, $\ell_{c r}$ is the critical relative angular momentum leading to impurity capture, and $\kappa=\left(2 \mu E / \hbar^{2}\right)^{1 / 2}$ with $\mu$ being the reduced mass of the system. For a given energy, $\ell_{c r}$ is determined by carrying out a series of simulations with varying impact parameters. This procedure was implemented for $\mathrm{Cs}^{279}$ and recently also for Xe.231

For a Xe atom at $v_{0}=200 \mathrm{~m} / \mathrm{s}$, the impact parameter leading to its capture is approximately $20.5 \AA$, which can be compared with the sharp-density radius of the ${ }^{4} \mathrm{He}_{1000}$ droplet, $22.2 \AA$. Hence, at thermal velocities the calculated cross-section for Xe capture is close to the geometrical cross-section of the droplet itself. The angular momentum of the impinging Xe at $v_{0}=200 \mathrm{~m} / \mathrm{s}$ with an impact parameter of $22.2 \AA$ is $917 \hbar$. This collision was simulated for $200 \mathrm{ps}^{231}$ and it was found that $15 \mathrm{He}$ atoms were ejected during this time period, of which 5 remained attached to the Xe atom. After the collision, the $\mathrm{Xe}+{ }^{4} \mathrm{He}_{5}$ complex carries 
away $522 \hbar$ angular momentum units, while some $95 \hbar$ units are deposited into the droplet as vortex loops and capillary waves. The remaining angular momentum is taken away by the promptly emitted He atoms.

\section{Droplets hosting vortices}

Recently, an experimental technique for determining the size of large He droplets $\left(N_{4}>\right.$ $10^{5}$ ) has been introduced ${ }^{282}$ that is based on the attenuation of a continuous droplet beam through collisions with Ar atoms at room temperature. The pickup chamber of the droplet beam apparatus is filled with argon gas and the helium droplets are subjected to multiple isotropic collisions with Ar atoms on their way to the detection chamber; large helium droplets could also be doped by impurities using this approach. The experimental situation for large superfluid He droplets is discussed in Ref. 283.

This method has been instrumental for visualizing quantised vortex arrays in large helium droplets $\left(10^{8}-10^{11}\right.$ atoms $)$ doped with Xe atoms and clusters. ${ }^{29|116| 117}$ Although experimental data for Ar was also recorded, the analysis has been limited so far to Xe because of the higher sensitivity in coherent $\mathrm{x}$-ray diffractive imaging. These experiments have motivated a series of TDDFT simulations on the impurity capture process by vortex lines at impact velocities relevant to the experimental conditions.

The capture of thermal Ar and Xe atoms by a linear vortex line hosted inside a ${ }^{4} \mathrm{He}_{1000}$ droplet has been recently studied by TDDFT!231 The vortex line was generated by the imprinting method described in Sec. IIIB and the perpendicular impurity-vortex impact took place on the equatorial plane of the droplet at $240(\mathrm{Xe})$ or $360 \mathrm{~m} / \mathrm{s}(\mathrm{Ar})$. In both cases, the impurity is 'captured' by the vortex line in the sense that, after a few hundred ps, it orbits around the vortex line and remains at a close distance from it. $\underline{50 \mid 231}$

The right panel of Fig. 39 shows snapshots of the collision process for $\operatorname{Ar}\left(v_{0}=360 \mathrm{~m} / \mathrm{s}\right)$ with a ${ }^{4} \mathrm{He}_{1000}$ droplet hosting a single vortex line. These data, together with those in the left panel, show the corresponding Ar atom trajectories with and without a vortex line. The first turning point of $\mathrm{Ar}$ in a vortex-free droplet is reached at $68 \mathrm{ps}$, and is located close to the droplet surface. The equivalent configuration in the presence of a vortex line is shown by the snapshot at $74 \mathrm{ps}$. The vortex-free configurations at 311 and 410 ps correspond to the $4^{\text {th }}$ and $5^{\text {th }}$ turning points of the Ar trajectory. Similar configurations hosting a vortex line are 
shown in the right panel, which demonstrates that the Ar atom trajectory becomes localised in the immediate neighborhood of the vortex line due to the impurity-vortex binding. Note that additional sources of dissipation (e.g. viscosity), which are not included in TDDFT, may only enhance this localisation process.

\section{P. Liquid helium on nanostructured surfaces}

As a consequence of the extremely weak He-He interaction, it is expected that liquid helium interacts strongly with almost any substrate and wet the surface such that the vapor and the substrate are not in contact. However, since the interaction of helium with alkali atoms is even weaker than the He-He interaction, they might represent a notable exception to this rule. Indeed, while liquid ${ }^{3} \mathrm{He}$ wets any substrate, it has been shown that liquid ${ }^{4} \mathrm{He}$ does not wet surfaces made of heavy alkali metals such as Cs at $T=0.2284 \sqrt[285]{ }$

The wetting properties of ${ }^{4} \mathrm{He}$ on the surface of heavy alkali metals have been studied in the past by using a $T$-dependent free-energy density functional, which describes the surface properties of liquid ${ }^{4} \mathrm{He}$ accurately in the $0<T<3 \mathrm{~K}$ temperature range ${ }^{51}$ The resulting liquid structure on the Cs surface was elucidated, providing both the $T$-dependence of the contact angle and the wetting temperature, which are in good agreement with experiments.

The most recent research employing DFT to study the adsorption of helium samples on various substrates has been reviewed in Ref. 286. The issues addressed in this review include the deposition and spreading of helium droplets on flat alkali metal surfaces; the determination of isotherms; the construction of the phase diagram of helium on such substrates; the adsorption of helium on spherical and cylindrical surfaces; the filling of wedges and the filling/emptying transitions at $T=0$ taking place at fixed values of the wedge opening angle, $\frac{\sqrt{287}}{2}$ the filling of infinite polygonal pores, and the adsorption on planar surfaces structured with an array of parabolic nanocavities. In particular, the prewetting line and isotherms for helium-Cs adsorbed on nano-patterned surfaces with parabolic cavities were studied by the finite temperature DFT approach. $\stackrel{51 / 272}{ }$ The results obtained for Cs surfaces (non-wettable) were compared with the corresponding planar $\mathrm{Na}$ (wettable at $T=0$ ) and nano-patterned $\mathrm{Na}$ surfaces.

To illustrate how wetting of a patterned surface proceeds, we show some configurations in Fig. 41 for the wetting sequence of a $\mathrm{Na}$ surface at $T=0.5 \mathrm{~K}$, from low to higher 

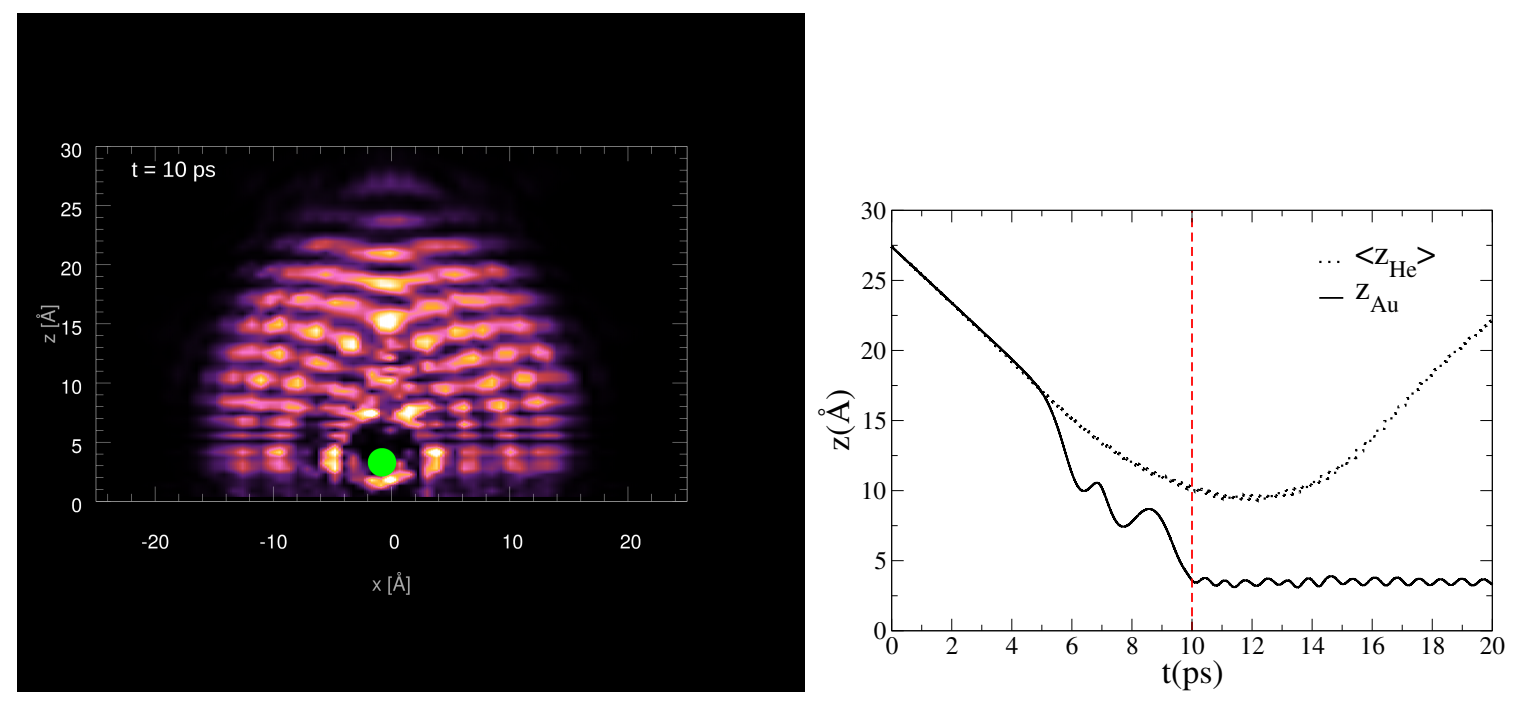

FIG. 42. $\mathrm{Au} @{ }^{4} \mathrm{He}_{300}$ landing on rutile $\mathrm{TiO}_{2}(110)$-surface at $200 \mathrm{~m} / \mathrm{s}$. (a) Droplet density on a plane perpendicular to the surface at $t=10 \mathrm{ps}$. Bright spots correspond to high helium densities and the $\mathrm{Au}$ atom is represented by a green dot. (b) Position of the Au atom (solid line) and the COM of the helium droplet (dotted line) with respect to the surface plane. The vertical dashed line indicates the time corresponding to the snapshot shown in (a). 288

coverage. The Na surface is patterned with an array of periodically repeated parabolic cavities of nanoscopic size, and the He coverage is increased continuously. Panel I shows the low coverage phase up to the complete filling of each heliophilic parabolic cavity; in panel II, droplets grow on the flat region between the periodically repeated cavities as coverage increases; in panel III the drops on the flat region have merged together and only a ringshaped region around the edge of the cavity remains covered by a thin helium film. Finally, complete filling of the annular region occurs (panels IV and V); at relatively higher coverages, a very thick film made of several monolayers covers the surface and grows continuously with increasing coverage (panel VI). 


\section{Q. Soft-landing of helium droplets}

The time-dependent process of helium droplet landing on solid surfaces, which bears similarities with the surface wetting process discussed in the previous section, has been studied by TDDFT. In addition to contributing to the understanding of the basic physics governing such a collision event, the species formed inside the droplets can be gently deposited onto the surface (soft-landing). With the recent general interest in nano-sciences and nano-technology, this approach has received significant attention because it can potentially be used to extract the nanostructures formed inside helium droplets for practical applications. 1491289 For example, metallic nanoclusters and nanowires, which are predicted to be excellent catalysts due to their large surface area, could be produced by using this technique. For a review on soft-landing, see Ref. 290.

Soft-landing of metal (e.g. Ag) doped large helium droplets has also been instrumental for the discovery of vortex lines inside the droplets. 240|241|291 The experiments revealed the presence of linear structures on the deposition surface along which a series of nanoparticles were distributed. The observed linear geometries have been suggested to arise either from vortex mediated nanowire assembly (and subsequent decomposition) or from direct trapping of multiple metal nanoparticles on a vortex line. Both mechanisms require the presence of quantized vortices inside the droplets.

The first TDDFT studies for the softlanding of pure helium droplets on surfaces with potential technological interest have been carried recently. ${ }^{292 \mid 293}$ The model system considered consisted of a ${ }^{4} \mathrm{He}_{300}$ droplet traveling at $200 \mathrm{~m} / \mathrm{s}$ towards a $\mathrm{TiO}_{2}$ (110)-surface. To identify the possible quantum effects, both classical molecular dynamics and TDDFT simulations were carried out. In contrast to the classical results, which show the helium droplet splashing off the surface on impact, the TDDFT evolution leads to the spreading of the liquid on the surface. This thin film formation is a process similar to the surface wetting described in the previous section. In addition to $\mathrm{TiO}_{2}$, a graphite sheet was also considered as a target.293 Despite the omission of thermal effects and the small droplet size considered, these studies have provided a solid starting point for simulating helium droplet mediated deposition of metallic clusters on substrates.

Deposition of an $\mathrm{Au}$ atom embedded inside a ${ }^{4} \mathrm{He}_{300}$ droplet on a $\mathrm{TiO}_{2}$ (110)-surface was addressed in Ref. 288. This was the first theoretical study that considered the experimentally 
studied landing process as described e.g. in Refs. 149, 289, and 294, The outcome of the simulation for an $\mathrm{Au} @{ }^{4} \mathrm{He}_{300}$ complex at $200 \mathrm{~m} / \mathrm{s}$, with COM initially located at $27.4 \AA$ from the surface, is shown in Fig. 42, As shown in the figure, the Au atom initially follows the droplet, then begins to oscillate back and forth inside it, and finally becomes trapped inside the $\mathrm{Au}-\mathrm{TiO}_{2}$ surface potential minimum. After ca. 10 ps, the atom keeps oscillating about the potential energy minimum until the end of the simulation (zero average acceleration). The spreading of the droplet on the surface was observed, but to a lesser degree than for the pure helium droplet. ${ }^{292}$ By comparing the results from TDDFT and classical calculations, it was concluded that the proper description of this ${ }^{4} \mathrm{He}$ droplet-assisted process must be carried out using quantum mechanical simulations.

\section{SUMMARY AND OUTLOOK}

The density functional approach offers a unique method to study both static and dynamic response of superfluid helium. In addition to atomic and molecular impurities, it can also be applied in its present form to model the interaction of nanometer-scale objects with the liquid. From the computational resource perspective, the method is easily applied to systems up to $100 \mathrm{~nm}$ in size in 3D, the main limitations being both computer time and memory requirements. The formulation allows for the description of both helium droplets and bulk liquid through suitable boundary conditions. With the recent improvements to the OT-DFT functional, strongly inhomogeneous snowball systems can now be modelled. Unlike QMC-based methods, DFT can yield real time quantum dynamics. The examples summarised in this review provide extensive evidence that the TDDFT approach is capable of reproducing the results from a wide range of time-resolved experiments, especially in superfluid helium droplets. It is the only method that allows this close interplay between theory and experiment in this field.

Despite the enormous success of OT-DFT, and DFT in general, there is still room for improvement in both accuracy and functionality. For example, just like any other DFTbased method, it is not straightforward to couple DFT to any degrees of freedom that follow traditional quantum mechanics. The often employed coupled quantum and OT-DFT equations presented in this review all either ignore the quantum correlations between the two subsystems or incorporate them in a phenomenological way into the interaction potential. 
In many systems, such as the electron bubble, this correlation may not be significant due to the large mass difference between the electron and helium. But it will arise, for example, in the treatment of molecular rotation in superfluid helium. Up to now the latter problem has only been addressed in terms of classical rotation and the associated moment of inertia, which is the likely origin of the remaining discrepancy between the OT-DFT calculations and experimental results (Sec. VK). A similar issue should arise in the treatment of molecular vibrations of impurities solvated in superfluid helium.

While PIMC calculations can elegantly model superfluid helium at finite temperatures, OT-DFT in its basic formulation is restricted to $0 \mathrm{~K}$. The static liquid response up to $3 \mathrm{~K}$ temperature has been introduced into OT-DFT, but it is still missing dynamic contributions such as the viscous response. The viscous response can be included from continuum fluid mechanics (Navier-Stokes) into OT-DFT, but it is not a priori clear how it should behave at the typically observed rather wide gas-liquid interfaces around solvated impurities. In general, one possible strategy may be to follow the very successful Landau's two-fluid model and treat the superfluid and normal fractions in DFT separately.

Many elegant experiments have been carried out in bulk superfluid helium over the years, which would require at least a mesoscopic-size description of the system. Therefore, they are currently not accessible to the OT-DFT approach due to the limitations in current computational resources. In order to employ the DFT approach for such systems, new strategies are needed to reduce especially the memory requirements of the calculations. An obvious approach is to use any symmetry present in the system and formulate the problem in $1 \mathrm{D}$ or $2 \mathrm{D}$ rather than the full $3 \mathrm{D}$. However, often such symmetry is not present and furthermore, the numerical implementation of OT-DFT in reduced dimensions is sometimes far from trivial.

If a distant part of the system can be treated with a limited accuracy, multi-scale-type models could be developed to expand the spatial domain considered. Another option would be to vary the resolution of the spatial grid, which allows allocating more points to the regions of interest. However, all current numerical implementations of OT-DFT are restricted to uniform grids because they employ the finite difference approximation and the FFT algorithm for evaluating the non-linear potential. While the well-known finite element method could replace the finite difference approach, the heavily used FFT would still not be applicable for non-uniform grids. 
Notwithstanding the need for improvements, the applications of the current OT-DFT approach, especially in its time-dependent version, are noteworthy. Among the projects that are being conducted during the completion of this review, let us mention e.g. the desorption of intrinsic and extrinsic impurities from helium droplets; the description of soft-landing processes under conditions closer to the experimental situation; the multiple capture of impurities by droplets hosting vortex arrays, and the propagation of shock waves and solitons, and gas bubble dynamics and vortex nucleation in liquid helium.

Finally, let us indicate that the CSU at Northridge and Barcelona-Toulouse helium-DFT codes are available at the following repositories:

- CSU at Northridge He-DFT code:

https://sourceforge.net/projects/libgrid/

https://sourceforge.net/projects/libdft/

- BCN-TLS DFT He-code:

https://github.com/bcntls2016/4hedft

https://github.com/bcntls2016/4hedft-vortex

https://github.com/bcntls2016/4hetddft-isotropic

https://github.com/bcntls2016/4hetddft-anisotropic

\section{ACKNOWLEDGMENTS}

We would like to thank the many colleagues whose collaboration in the development of the DFT method, triggered in most cases by their experimental work, has made possible that the DFT approach has reached the level of sophistication and applicability this review is based on. This work has been performed under Grants No. FIS2014-52285-C2-1-P from DGI, Spain, and 2014SGR401 from Generalitat de Catalunya. MB thanks the Université Fédérale Toulouse Midi-Pyrénées for financial support during the completion of this work throughout the 'Chaires d'Attractivité 2014' Programme IMDYNHE. JE acknowledges financial support from NSF grant DMR-1205734.

1 D. R. Tilley and J. Tilley, Superfluidity and superconductivity (John Wiley \& Sons, New York, 1974). 
2 A. F. Borghesani, Ions and electrons in liquid helium (Oxford Science Publications, New York, 2007).

3 M. Hartmann, R. E. Miller, J. P. Toennies, and A. F. Vilesov, Phys. Rev. Lett. 75, 1566 (1995).

4 M. Hartmann, F. Mielke, J. P. Toennies, A. F. Vilesov, and G. Benedek, Phys. Rev. Lett. 76, $4560(1996)$.

5 K. K. Lehmann and G. Scoles, Science 279, 2065 (1998).

6 J. P. Toennies and A. F. Vilesov, Angew. Chem. Phys. 43, 2622 (2004).

7 M. Barranco, R. Guardiola, S. Hernández, R. Mayol, J. Navarro, and M. Pi, J. Low Temp. Phys. 142, 1 (2006).

8 K. Szalewicz, Int. Rev. Phys. Chem. 27, 273 (2006).

9 F. Stienkemeier and K. K. Lehmann, J. Phys. B 39, R127 (2006).

10 M. Y. Choi, G. E. Douberly, T. M. Falconer, W. K. Lewis, C. M. Lindsay, J. M. Merritt, P. L. Stiles, and R. E. Miller, Int. Rev. Phys. Chem. 25, 15 (2006).

11 J. Tiggesbäumker and F. Stienkemeier, Phys. Chem. Chem. Phys. 9, 4748 (2007).

12 A. Slenczka and J. P. Toennies, in Low Temperatures and Cold Molecules, edited by W. M. Smith (World Sci., Singapore, 2008), p. 345.

13 C. Callegari and W. E. Ernst, in Handbook of High Resolution Spectroscopy, Vol. 3 (Whiley, New York, 2011), p. 1551.

14 S. Yang and A. M. Ellis, Chem. Soc. Rev 42, 472 (2013).

15 M. Mudrich and F. Stienkemeier, Int. Rev. Phys. Chem. 33, 301 (2014).

16 J. P. Toennies, The Chemical physics of Atomic and Molecular Clusters, Course CVII, in Proceedings of the International School of Physics "Enrico Fermi" 1988 (North Holland, Amsterdam, 1990), p. 597.

17 K. B. Whaley, Int. Rev. Phys. Chem. 13, 41 (1994).

18 K. B. Whaley, Advances in Molecular Vibrations and Collision Dynamics 3, 397 (1998).

19 J. P. Toennies and A. F. Vilesov, Ann. Rev. Phys. Chem. 49, 1 (1998).

20 J. A. Northby, J. Chem. Phys. 115, 10065 (2001), Special Topic issue on Helium nanodroplets.

21 E. Krotscheck and J. Navarro, editors, Microscopic approaches to quantum liquids in confined geometries, Series on Advances in Quantum Many-Body Theory, Vol. 4 (World Scientific, Singapore, 2002).

22 M. P. de Lara-Castells, G. Delgado-Barrio, P. Villarreal, and A. O. Mitrushchenkov, J. Chem. 
Phys. 125, 221101 (2006).

23 M. P. de Lara-Castells, N. F. Aguirre, P. Villarreal, G. Delgado-Barrio, and A. O. Mitrushchenkov, J. Chem. Phys. 132, 194313 (2010).

24 N. F. Aguirre, P. Villarreal, G. Delgado-Barrio, A. O. Mitrushchenkov, and M. P. de LaraCastells, Phys. Chem. Chem. Phys. 15, 10126 (2013).

25 S. Stringari and J. Treiner, Phys. Rev. B 36, 8369 (1987).

26 S. Stringari and J. Treiner, J. Chem. Phys. 87, 5021 (1987).

27 F. Dalfovo, A. Lastri, L. Pricaupenko, S. Stringari, and J. Treiner, Phys. Rev. B 52, 1193 (1995).

28 M. P. Ziemkiewicz, D. M. Neumark, and O. Gessner, Int. Rev. Phys. Chem. 34, 239 (2015).

29 L. F. Gomez, K. R. Ferguson, J. P. Cryan, C. Bacellar, R. M. P. Tanyag, C. Jones, S. Schorb, D. Anielski, A. Belkacem, C. Bernando, R. Boll, J. Bozek, et al., Science 345, 906 (2014).

30 N. B. Brauer, S. Smolarek, E. Loginov, D. Mateo, A. Hernando, M. Pi, M. Barranco, W. J. Buma, and M. Drabbels, Phys. Rev. Lett. 111, 153002 (2013).

31 J. von Vangerow, F. Coppens, A. Leal, M. Pi, M. Barranco, N. Halberstadt, F. Stienkemeier, and M. Mudrich, J. Phys. Chem. Lett. 8, 307 (2017).

32 A. Leal, D. Mateo, A. Hernando, M. Pi, M. Barranco, A. Ponti, F. Cargnoni, and M. Drabbels, Phys. Rev. B 90, 224518 (2014).

33 S. L. Fiedel, D. Mateo, T. Aleksanyan, and J. Eloranta, Phys. Rev. B 86, 144522 (2012).

34 M. Barranco, F. Coppens, N. Halberstadt, A. Hernando, A. Leal, D. Mateo, R. Mayol, and M. Pi, Zero temperature DFT and TDDFT for ${ }^{4} H e:$ A short guide for practitioners (2017), https://github.com/bcntls2016/DFT-Guide/blob/master/dft-guide.pdf.

35 D. Mateo, M. Barranco, and J. Navarro, Phys. Rev. B 82, 134529 (2010).

36 R. J. Donnelly, J. A. Donnelly, and R. N. Hills, J. Low Temp. Phys. 44, 471 (1981).

37 P. Hohenberg and W. Kohn, Phys. Rev. 136, B864 (1964).

38 W. Kohn and L. J. Sham, Phys. Rev. 140, A1133 (1965).

39 E. Runge and E. K. U. Gross, Phys. Rev. Lett. 52, 997 (1984).

40 E. Madelung, Z. Phys. 40, 322 (1927).

41 F. Ancilotto, M. Barranco, and M. Pi, Phys. Rev. B 82, 014517 (2010).

42 E. Popov and J. Eloranta, J. Chem. Phys. 142, 204704 (2015).

43 D. Mateo, J. Eloranta, and G. A. Williams, J. Chem. Phys. 142, 064510 (2015). 
44 D. Mateo, A. Hernando, M. Barranco, E. Loginov, M. Drabbels, and M. Pi, Phys. Chem. Chem. Phys. 15, 18388 (2013).

45 N. G. Berloff and P. H. Roberts, J. Phys. A 34, 81 (2001).

46 D. Mateo, D. Jin, M. Barranco, and M. Pi, J. Chem. Phys. 134, 044507 (2011).

47 D. Jin and H. J. Maris, J. Low Temp. Phys. 158, 317 (2010).

48 D. Jin and W. Guo, Phys. Rev. B 82, 094524 (2010).

49 D. Jin, W. Guo, W. Wei, and H. J. Maris, J. Low Temp. Phys. 158, 307 (2010).

50 I. A. Pshenichnyuk and N. G. Berloff, Phys. Rev. B 94, 184505 (2016).

51 F. Ancilotto, F. Faccin, and F. Toigo, Phys. Rev. B 62, 17035 (2000).

52 T. Biben and D. Frenkel, J. Phys. Cond. Matt. 14, 9077 (2002).

53 D. Jin and W. Guo, J. Chem. Phys. 136, 244510 (2012).

54 M. Barranco, M. Pi, S. M. Gatica, E. S. Hernández, and J. Navarro, Phys. Rev. B 56, 8997 (1997).

55 A. Leal, D. Mateo, M. Pi, M. Barranco, and J. Navarro, J. Chem. Phys. 139, 174308 (2013).

56 A. Hernando, R. Mayol, M. Pi, M. Barranco, F. Ancilotto, O. Bünermann, and F. Stienkemeier, J. Phys. Chem. A 111, 7303 (2007).

57 E. S. Hernández and J. Navarro, in Microscopic approaches to quantum liquids in confined geometries, edited by E. Krotscheck and J. Navarro (World Sci, Singapore, 2002), p. 261.

58 M. Abad, M. Guilleumas, R. Mayol, M. Pi, and D. M. Jezek, Phys. Rev. A 81, 043619 (2010).

59 F. Ancilotto, M. Barranco, J. Navarro, and M. Pi, J. Low Temp. Phys. 185, 26 (2016).

60 F. Ancilotto, M. Barranco, F. Caupin, R. Mayol, and M. Pi, Phys. Rev. B 72, 214522 (2005).

61 F. Caupin, F. Ancilotto, M. Barranco, R. Mayol, and M. Pi, J. Low Temp. Phys. 148, 731 (2007).

62 F. Ancilotto, M. Pi, R. Mayol, M. Barranco, and K. K. Lehmann, J. Phys. Chem. A 111, 12695 (2007).

63 R. P. Feynman and M. Cohen, Phys. Rev. 102, 1189 (1956).

64 M. R. Gibbs, K. H. Andersen, W. G. Stirling, and H. Schober, J. Phys.: Condens. Matter 11, 603 (1999).

65 D. J. Thouless, Ann. Phys. (N.Y.) 52, 403 (1969).

66 L. Giacomazzi, F. Toigo, and F. Ancilotto, Phys. Rev. B 67, 104501 (2003).

67 L. Lehtovaara, T. Kiljunen, and J. Eloranta, J. Comput. Phys. 194, 78 (2004). 
L. Lehtovaara, J. Toivanen, and J. Eloranta, J. Comput. Phys. 221, 148 (2007).

69 M. Pi, R. Mayol, A. Hernando, M. Barranco, and F. Ancilotto, J. Chem. Phys. 126, 244502 (2007).

70 M. Frigo and S. G. Johnson, Proc. IEEE 93, 216 (2005).

71 F. Ancilotto, M. Pi, and M. Barranco, Phys. Rev. B 91, 100503(R) (2015).

72 F. Dalfovo, R. Mayol, M. Pi, and M. Barranco, Phys. Rev. Lett 85, 1028 (2000).

73 F. Ancilotto, M. Pi, and M. Barranco, Phys. Rev. B 90, 174512 (2014).

74 R. J. Donnelly, Quantized vortices in helium II, Cambridge Studies in Low Temperature Physics, Vol. 3 (Cambridge University Press, Cambridge, U.K., 1991).

75 F. Dalfovo and S. Stringari, Phys. Rev. A 53, 2477 (1996).

76 J. von Vangerow, A. Stieg, F. Stienkemeier, M. Mudrich, A. Leal, D. Mateo, A. Hernando, M. Barranco, and M. Pi, J. Phys. Chem. A 118, 6604 (2014).

77 D. Mateo, A. Leal, A. Hernando, M. Barranco, M. Pi, F. Cargnoni, M. Mella, X. Zhang, and M. Drabbels, J. Chem. Phys. 140, 131101 (2014).

78 A. I. Krylov, Annu. Rev. Phys. Chem. 59, 433 (2008).

79 R. E. Wyatt, Quantum Dynamics with Trajectories (Springer, New York, 2005).

80 A. Hernando, M. Barranco, M. Pi, E. Loginov, M. Langlet, and M. Drabbels, Phys. Chem. Chem. Phys. 14, 3996 (2012).

81 F. O. Ellison, J. Am. Chem. Soc. 85, 3540 (1963).

82 A. Hernando, M. Barranco, R. Mayol, M. Pi, and M. Krośnicki, Phys. Rev. B 77, 024513 (2008).

83 J. Eloranta, J. Ahokas, and H. Kunttu, J. Chem. Phys. 114, 7144 (2001).

84 Z. J. Jakubek, Q. Hui, and M. Takami, Phys. Rev. Lett. 79, 629 (1997).

85 A. Nakayama and K. Yamashita, J. Chem. Phys. 114, 780 (2001).

86 A. Leal, X. Zhang, M. Barranco, F. Cargnoni, A. Hernando, D. Mateo, M. Mella, M. Drabbels, and M. Pi, J. Chem. Phys. 144, 094302 (2016).

87 M. Mella and F. Cargnoni, J. Phys. Chem. A 118, 6473 (2014).

88 A. Ralston and H. S. Wilf, Mathematical methods for digital computers (John Wiley and Sons, New York, 1960).

89 W. H. Press, S. A. Teukolsky, W. T. Vetterling, and B. P. Flannery, Numerical Recipes (Cambridge University Press, Cambridge, 1992). 
90 D. Nettels, A. Hofer, P. Moroshkin, R. Müller-Siebert, S. Ulzega, and A. Weis, Phys. Rev. Lett. 94, 063001 (2005).

91 X. Buelna, A. Freund, D. Gonzalez, E. Popov, and J. Eloranta, J. Phys. Chem. B 120, 11010 (2016).

92 M. Barranco, J. Eloranta, A. Hernando, and M. Pi, unpublished (2016) .

93 S. H. Patil, J. Chem. Phys. 94, 8089 (1991).

94 J. Pascale, Phys. Rev. A 28, 632 (1983).

95 F. Stienkemeier, F. Meier, and H. O. Lutz, Eur. Phys. J. D 9, 313 (1999).

96 D. Mateo, A. Hernando, M. Barranco, R. Mayol, and M. Pi, Phys. Rev. B 83, 174505 (2011).

97 A. Hernando, M. Barranco, R. Mayol, M. Pi, F. Ancilotto, O. Bünermann, and F. Stienkemeier, J. Low Temp. Phys. 158, 105 (2010).

98 D. Tannor, Introduction to Quantum mechanics: A Time-Dependent Perspective (University Science Books, Sausalito, California (USA), 2007).

99 A. Hernando, R. Mayol, M. Pi, M. Barranco, I. S. K. Kerkines, and A. Mavridis, Int. J. of Quantum Chem. 111, 400 (2011).

100 J. Eloranta, H. Y. Seferyan, and V. A. Apkarian, Chem. Phys. Lett. 396, 155 (2004).

101 F. Ancilotto, P. B. Lerner, and M. W. Cole, J. Low Temp. Phys. 101, 1123 (1995).

102 F. Stienkemeier, J. Higgins, C. Callegari, S. I. Kanorsky, W. E. Ernst, and G. Scoles, Z. Phys. D 38, 253 (1996).

103 O. Bünermann, G. Droppelmann, A. Hernando, R. Mayol, and F. Stienkemeier, J. Phys. Chem. A 111, 12684 (2007).

104 F. Lackner, J. Poms, G. Krois, J. V. Pototschnig, and W. E. Ernst, J. Phys. Chem. A 117, 11866 (2013).

105 C. Callegari and F. Ancilotto, J. Phys. Chem. A 115, 6789 (2011).

106 A. Pifrader, O. Allard, G. Auböck, C. Callegari, W. E. Ernst, R. Hubert, and F. Ancilotto, J. Chem. Phys. 133, 164502 (2010).

107 E. Loginov, C. Callegari, F. Ancilotto, and M. Drabbels, J. Phys. Chem. A 115, 6779 (2011).

108 L. Fechner, B. Grüner, A. Sieg, C. Callegari, F. Ancilotto, F. Stienkemeier, and M. Mudrich, Phys. Chem. Chem. Phys. 14, 3843 (2012).

109 A. Hernando, J. A. Beswick, and N. Halberstadt, J. Chem. Phys. 139, 221102 (2013).

110 E. Loginov, A. Hernando, J. A. Beswick, N. Halberstadt, and M. Drabbels, J. Phys. Chem. A 
119, $6033(2015)$.

111 B. Gervais, D. Zanuttini, and J. Douady, J. Chem. Phys. 144, 194307 (2016).

112 J. D. Close, F. Federman, K. Hoffmann, and N. Quass, J. Low Temp. Phys. 111, 661 (1998).

113 D. Mateo, A. Hernando, M. Barranco, and M. Pi, J. Low Temp. Phys. 158, 397 (2010).

114 F. Stienkemeier, F. Meier, and H. O. Lutz, J. Chem. Phys. 107, 10816 (1997).

115 F. Ancilotto, M. Barranco, and M. Pi, Phys. Rev. Lett. 91, 105302 (2003).

116 C. F. Jones, C. Bernando, R. M. P. Tanyag, C. Bacellar, K. R. Ferguson, L. F. Gomez, D. Anielski, A. Belkacem, R. Boll, J. Bozek, S. Carron, J. Cryan, et al., Phys. Rev. B 93, 180510(R) (2016).

117 C. Bernando, R. Mayro, P. Tanyag, C. Jones, C. Bacellar, M. Bucher, K. R. Ferguson, D. Rupp, M. P. Ziemkiewicz, L. F. Gomez, A. S. Chatterley, T. Gorkhover, et al., Phys. Rev. B 95, $064510(2017)$.

118 Y. Moriwaki and N. Morita, Eur. Phys. J. D 33, 323 (2005).

119 A. Hernando, M. Barranco, R. Mayol, M. Pi, and F. Ancilotto, Phys. Rev. B 78, 184515 (2008).

120 M. Mella, G. Calderoni, and F. Cargnoni, J. Chem. Phys. 123, 054328 (2005).

121 J. Navarro, D. Mateo, M. Barranco, and A. Sarsa, J. Chem. Phys. 136, 054301 (2012).

122 E. Krotscheck and R. E. Zillich, J. Chem. Phys. 145, 244317 (2016).

123 J. Reho, U. Merker, M. R. Radcliff, K. K. Lehmann, and G. Scoles, J. Chem. Phys. 112, 8409 (2000).

124 A. Przystawik, S. Göde, T. Döppner, J. Tiggesbäumker, and K-H. Meiwes-Broer, Phys. Rev. A 78, 021202(R) (2008).

125 Y. Ren and V. V. Kresin, Phys. Rev. A 76, 043204 (2007).

126 M. Lewerenz, B. Schilling, and J. P. Toennies, J. Chem. Phys. 102, 8191 (1995).

127 E. B. Gordon, J. Low Temp. Phys. 30, 756 (2004).

128 J. Eloranta, Phys. Rev. B 77, 134301 (2008).

129 A. W. Hauser, A. Volk, P. Thaler, and W. E. Ernst, Phys. Chem. Chem. Phys. 17, 10805 (2015).

130 J. Eloranta, Low Temp. Phys. 37, 384 (2011).

131 S.Göde, R. Irsing, J. Tiggesbäumker, and K-H. Meiwes-Broer, New J. Phys. 15, 015026 (2013).

132 A. Hernando, F. Ancilotto, M. Barranco, R. Mayol, and M. Pi, J. of Physics: Conf. Ser. 150, 032003 (2009). 
E. Loginov, L. F. Gomez, N. Chiang, A. Halder, N. Guggemos, V. V. Kresin, and A. F. Vilesov, Phys. Rev. Lett. 106, 233401 (2011).

134 E. Lugovoj, J. P. Toennies, and A. F. Vilesov, J. Chem. Phys. 112, 8217 (2000).

135 G. E. Douberly, P. L. Stiles, R. E. Miller, R. Schmied, and K. K. Lehmann, J. Phys. Chem. A 114, $3391(2010)$.

136 A. Masson, M. Briant, A. Hernando, N. Halberstadt, J.-M. Mestdagh, and M.-A. Gaveau, J. Chem. Phys. 137, 184310 (2012).

137 A. Hernando, A. Masson, M. Briant, J.-M. Mestdagh, M.-A. Gaveau, and N. Halberstadt, J. Chem. Phys. 137, 184311 (2012).

138 J. Poms, A. W. Hauser, and W. E. Ernst, Phys. Chem. Chem. Phys. 14, 15165 (2012).

139 M. Renzler, M. Daxner, L. Kranabetter, A. Kaiser, A. W. Hauser, W. E. Ernst, A. Lindinger, and R. E. Zillich, J. Chem. Phys. 145, 181101 (2016).

140 J. Höller, E. Krotscheck, and R. E. Zillich, Eur. Phys. J. D 69, 198 (2015).

141 C. Stark and V. V. Kresin, Phys. Rev. B 81, 085401 (2010).

142 L. An der Lan, P. Bartl, C. Leidlmair, H. Schöbel, R. Jochum, S. Denifl, T. D. Märk, A. M. Ellis, and P. Scheier, J. Chem. Phys. 135, 044309 (2011).

143 F. Calvo, Phys. Rev. B 95, 035429 (2017).

144 J. Höller, E. Krotscheck, and R. E. Zillich, Eur. Phys. J. D 68, 372 (2014).

145 Y. Kwon and H. Shin, Phys. Rev. B 82, 172506 (2010).

146 H. Shin and Y. Kwon, J. Chem. Phys. 136, 064514 (2012).

147 D. Bonhommeau, P. T. Lake Jr., C. L. Quiniou, M. Lewerenz, and N. Halberstadt, J. Chem. Phys. 126, 051104 (2007).

148 D. Bonhommeau, M. Lewerenz, and N. Halberstadt, J. Chem. Phys. 128, 054302 (2008).

149 V. Mozhayskiy, M. N. Slipchenko, V. K. Adamchuk, and A. F. Vilesov, J. Chem. Phys. 127, 094701 (2007).

150 E. Loginov, L. F. Gomez, and A. F. Vilesov, Phys. Chem. A 117, 11771 (2013).

151 A. W. Hauser and M. P. de Lara-Castells, J. Phys. Chem. Lett. 7, 4929 (2016).

152 A. W. Hauser, A. O. Mitrushchenkov, and M. P. de Lara-Castells, J. Phys. Chem. C 121, 3807 (2017).

153 B. E. Clements, E. Krotscheck, and H. J. Lauter, Phys. Rev. Lett. 70, 1287 (1993).

154 E. S. Hernández, J. Low Temp. Phys. 162, 583 (2011). 
155 S. Grebenev, J. P. Toennies, and A. F. Vilesov, Science 279, 2083 (1998).

156 O. Bünermann, M. Dvorak, F. Stienkemeier, A. Hernando, R. Mayol, M. Pi, M. Barranco, and F. Ancilotto, Phys. Rev. B 79, 214511 (2009).

157 R. Guardiola, J. Navarro, D. Mateo, and M. Barranco, J. Chem. Phys. 131, 174110 (2009).

158 L. S. Cederbaum, J. Zobeley, and F. Tarantelli, Phys. Rev. Lett. 79, 4778 (1997).

159 N. V. Kryzhevoi, D. Mateo, M. Pi, M. Barranco, and L. S. Cederbaum, Phys. Chem. Chem. Phys. 15, 18167 (2013).

160 N. V. Kryzhevoi, V. Averbukh, and L. S. Cederbaum, Phys. Rev. B 76, 094513 (2007).

161 M. Shcherbinin, A. C. LaForge, V. Sharma, M. Devetta, R. Richter, R. Moshammer, T. Pfeifer, and M. Mudrich, submitted to Phys. Rev. Lett. (2017).

162 M. Guilleumas, F. Garcias, M. Barranco, M. Pi, and E. Suraud, Z. Phys. D 26, 385 (1993).

163 D. Mateo, M. Barranco, R. Mayol, and M. Pi, Eur. Phys. J. D 52, 63 (2009).

164 D. Mateo, M. Barranco, R. Mayol, J. Navarro, and M. Pi, J. of Physics: Conf. Ser. 150, 032051 (2009).

165 B. G. Sartakov, J. P. Toennies, and A. F. Vilesov, J. Chem. Phys. 136, 134316 (2012).

166 D. Mateo, M. Pi, J. Navarro, and J. P. Toennies, J. Chem. Phys. 138, 044321 (2013).

167 N. Pörtner, J. P. Toennies, A. F. Vilesov, G. Benedek, and W. Hizhnyakov, Europhys. Lett. 88, 26007 (2009).

168 G. Benedek and V. Hizhnyakov, Chem. Phys. Lett. 548, 17 (2012).

169 J. Barragán, D. Mateo, M. Pi, F. Salvat, M. Barranco, and H. J. Maris, J. Low Temp. Phys. 171, 171 (2013).

170 M. Barranco, M. Guilleumas, M. Pi, and D. M. Jezek, in Microscopic approaches to quantum liquids in confined geometries, edited by E. Krotscheck and J. Navarro (World Sci, Singapore, 2002), p. 319.

171 S. Balibar, J. Low Temp. Phys. 129, 363 (2002).

172 H. J. Maris, J. Phys. Soc. Jpn. 77, 111008 (2008).

173 B. Tabbert, H. Günther, and G. zu Putlitz, J. Low Tem. Phys. 109, 653 (1997).

174 K. Coutinho, S. Canuto, P. K. Mukherjee, and B. Fricke, in Advances in the Theory of Atomic and Molecular Systems: Dynamics, Spectroscopy, Clusters, and Nanostructures, edited by P. Piecuch, J. Maruani, G. Delgado-Barrio, and S. Wilson (Springer Netherlands, Dordrecht, 2009), p. 183. 
175 H. J. Maris, J. Low Temp. Phys. 173, 78 (2000).

176 W. Wei, Z. Xie, L. N. Cooper, G. M. Seidel, and H. J. Maris, J. Low Temp. Phys. 178, 78 (2015).

177 J. Tempere, I. F. Silvera, and J. T. Devreese, Sur. Sci. Rep 62, 159 (2007).

178 W. Guo, D. Jin, and H. J. Maris, Phys. Rev. B 78, 014511 (2008).

179 J. Fang, J. Tempere, and I. F. Silvera, J. Low Temp. Phys. 187, 54 (2017).

180 L. Lehtovaara and J. Eloranta, J. Low Temp. Phys. 148, 43 (2007).

181 J. Eloranta and V. A. Apkarian, J. Chem. Phys. 117, 10139 (2002).

182 V. Grau, M. Barranco, R. Mayol, and M. Pi, Phys. Rev. B 73, 064502 (2006).

183 C. C. Grimes and G. Adams, Phys. Rev. B 45, 2305 (1992).

184 J. Classen, C.-K. Su, M. Mohazzab, and H. J. Maris, Phys. Rev. B 57, 3000 (1998).

185 M. Pi, M. Barranco, R. Mayol, and V. Grau, J. Low Temp. Phys. 139, 397 (2005).

186 D. Mateo, M. Pi, and M. Barranco, Phys. Rev. B 81, 174510 (2010).

187 D. Konstantinov and H. J. Maris, Phys. Rev. Lett. 90, 025302 (2003).

188 H. J. Maris, A. Ghosh, D. Konstantinov, and M. Hirsch, J. Low Temp. Phys. 134, 227 (2004).

189 F. Ancilotto, F. Dalfovo, L. P. Pitaevskii, and F. Toigo, Phys. Rev. B 71, 104530 (2005).

190 G. G. Nancolas, T. Ellis, P. McClintock, and R. M. Bowley, Nature 316, 797 (1985).

191 S. Paolini, F. Ancilotto, and F. Toigo, J. Chem. Phys. 126, 124317 (2007).

192 P. Claas, S. O. Mende, and F. Stienkemeier, Rev. Sci. Instrum. 74, 4071 (2003).

193 A. Braun and M. Drabbels, Phys. Rev. Lett. 93, 253401 (2004).

194 X. Zhang and M. Drabbels, J. Chem. Phys. 137, 051102 (2012).

195 D. E. Galli, D. M. Ceperley, and L. Reatto, J. Phys. Chem. A 115, 7300 (2011).

196 M. Rossi, M. Verona, D. E. Galli, and L. Reatto, Phys. Rev. B 69, 212510 (2004).

197 L. An der Lan, P. Bartl, C. Leidlmair, R. Jochum, S. Denifl, O. Echt, and P. Scheier, Chem. Eur. J. 18, 4411 (2012).

198 P. Bartl, C. Leidlmair, S. Denifl, P. Scheier, and O. Echt, J. Phys. Chem. A 118, 8050 (2014).

199 T. Döppner, T. Diederich, A. Przystawik, N. X. Truong, T. Fennel, J. Tiggesbäumker, and K. Meiwes-Broer, Phys. Chem. Chem. Phys. 9, 4639 (2007).

200 P. Slavíček and M. Lewerenz, Phys. Chem. Chem. Phys. 12, 1152 (2010).

201 N. Issaoui, K. Abdessalem, H. Ghalla, S. J. Yaghmour, F. Calvo, and B. Ouija, J. Chem. Phys. 141, $174316(2014)$. 
202 E. Loginov and M. Drabbels, Phys. Rev. Lett. 106, 083401 (2011).

203 S. Müller, M. Mudrich, and F. Stienkemeier, J. Chem. Phys. 131, 044319 (2009).

204 M. Theisen, F. Lackner, and W. E. Ernst, J. Chem. Phys. 135, 074306 (2011).

205 M. Theisen, F. Lackner, and W. E. Ernst, Phys. Chem. Chem. Phys. 12, 14861 (2010).

206 H. J. Reyher, H. Bauer, C. Huber, R. Mayer, A. Schafer, and A. Winnacker, Phys. Lett. A 115, 238 (1986).

207 W. S. Dennis, E. Durbin, Jr., W. A. Fitzsimmons, O. Heybey, and G. K. Walters, Phys. Rev. Lett. 23, 1083 (1969).

208 J. C. Hill, O. Heybey, and G. K. Walters, Phys. Rev. Lett. 26, 1213 (1971).

209 D. E. Zmeev, F. Pakpour, P. M. Walmsley, A. I. Golov, W. Guo, D. N. McKinsey, G. G. Ihas, P. V. E. McClintock, S. N. Fisher, and W. F. Vinen, Phys. Rev. Lett. 110, 175303 (2013).

210 A. V. Benderskii, J. Eloranta, R. Zadoyan, and V. A. Apkarian, J. Chem. Phys. 117, 1201 (2002).

211 J. Eloranta and V. A. Apkarian, J. Chem. Phys. 115, 752 (2001).

212 N. Bonifaci, F. Aitken, V. M. Atrazhev, S. L. Fiedler, and J. Eloranta, Phys. Rev. A 85, 042706 (2012).

213 S. L. Fiedler and J. Eloranta, J. Low Temp. Phys. 174, 269 (2014).

214 N. Bonifaci, Z. Li, J. Eloranta, and S. L. Fiedler, J. Phys. Chem. A 120, 9019 (2016).

215 J. Eloranta, N. Schwentner, and V. A. Apkarian, J. Chem. Phys. 116, 4039 (2002).

216 A. P. Hickman and N. F. Lane, Phys. Rev. B 12, 3705 (1975).

217 F. J. Soley and W. A. Fitzsimmons, Phys. Rev. Lett. 32, 988 (1974).

218 J. Eloranta, Chem. Phys. 332, 304 (2007).

219 D. Mateo, F. Gonzalez, and J. Eloranta, J. Phys. Chem. A 119, 2262 (2015).

220 M. Buzzacchi, D. E. Galli, and L. Reatto, Phys. Rev. B 64, 094512 (2001).

221 F. Aitken, N. Bonifaci, K. von Haeften, and J. Eloranta, J. Chem. Phys. 145, 044105 (2016).

222 A. Bartelt, J. D. Close, F. Federmann, N. Quaas, and J. P. Toennies, Phys. Rev. Lett. 77, 3525 (1996).

223 S. Grebenev, M. Hartmann, A. Lindinger, N. Pörtner, B. Sartakov, J. P. Toennies, and A. F. Vilesov, Physica B 280, 65 (2000).

224 J. Tang, Y. Xu, A. R. W. McKellar, and W. Jäger, Science 297, 2030 (2002).

225 Y. Xu and W. Jäger, J. Chem. Phys. 119, 5457 (2003). 
226 A. R. W. McKellar, Y. Xu, and W. Jäger, J. Phys. Chem. A 111, 7329 (2007).

227 L. A. Surin, A. V. Potapov, B. S. Dumesh, S. Schlemmer, Y. Xu, P. L. Raston, and W. Jäger, Phys. Rev. Lett. 101, 233401 (2008).

228 P. Sindzingre, M. L. Klein, and D. M. Ceperley, Phys. Rev. Lett. 63, 1601 (1989).

229 E. Krotscheck and R. E. Zillich, J. Chem. Phys. 115, 10161 (2001).

230 M. Greiner, O. Mandel, T. Esslinger, T. W. Hänsch, and I. Bloch, Nature 415, 39 (2002).

231 F. Coppens, F. Ancilotto, M. Barranco, N. Halberstadt, and M. Pi 2017 submitted to Phys. Chem. Chem. Phys.

232 A. Ghosh and H. J. Maris, Phys. Rev. B 72, 054512 (2005).

233 J. W. P. Pratt and J. W. Zimmermann, Phys. Rev. 177, 412 (1969).

234 K. DeConde, G. A. Williams, and R. E. Packard, Phys. Rev. Lett. 33, 683 (1974).

235 T. Padmore, Phys. Rev. Lett. 28, 469 (1972).

236 D. Mateo and J. Eloranta, J. Phys. Chem. A 118, 6407 (2014).

237 E. B. Gordon, A. V. Karabulin, A. A. Morozov, V. I. Matyushenko, V. D. Sizov, and I. I. Khodos, J. Phys. Chem. Lett. 5, 1072 (2014).

238 E. B. Gordon, A. V. Karabulin, V. I. Matyushenko, V. D. Sizov, and I. I. Khodos, Laser Phys. Lett. 12, 096002 (2015).

239 E. Popov, M. Mammetkuliyev, and J. Eloranta, J. Chem. Phys. 138, 204307 (2013).

240 L. F. Gomez, E. Loginov, and A. F. Vilesov, Phys. Rev. Lett. 108, 155302 (2012).

241 E. Latimer, D. Spence, C. Feng, A. Boatwright, A. M. Ellis, and S. Yang, Nano Lett. 14, 2902 (2014).

242 D. Spence, E. Latimer, C. Feng, A. Boatwright, A. M. Ellis, and S. Y. Yang, Phys. Chem. Chem. Phys. 16, 6903 (2014).

243 R. M. Ostermeier and W. I. Glaberson, J. Low Temp. Phys. 20, 159 (1975).

244 G. A. Williams and R. E. Packard, Phys. Rev. Lett. 35, 237 (1975).

245 P. E. Parks and R. J. Donnelly, Phys. Rev. Lett. 16, 45 (1966).

246 A. Volk, Ph. Thaler, D. Knez, A. W. Hauser, J. Steurer, W. Grogger, F. Hofer, and W. E. Ernst, Phys. Chem. Chem. Phys. 18, 1451 (2016).

247 G. A. Williams and R. E. Packard, Phys. Rev. Lett. 33, 280 (1974).

248 T. Zhang and S. W. Van Sciver, Nature Physics 1, 36 (2005).

249 G. P. Bewley, D. P. Lathrop, and K. R. Sreenivasan, Nature 441, 588 (2006). 
250 G. B. Hess, Phys. Rev. 161, 189 (1967).

251 S. Chandrasekhar, Proc. R. Soc. London, Ser. A 286, 1 (1965).

252 R. A. Brown and L. E. Scriven, Proc. R. Soc. London, Ser. A 371, 331 (1980).

253 D. A. Butts and D. S. Rokhsar, Nature 397, 327 (1999).

254 A. Vilà, M. González, and R. Mayol, J. Chem. Theory Comput. 11, 899 (2015).

255 A. Vilà, M. González, and R. Mayol, Phys. Chem. Chem. Phys. 18, 2409 (2016).

256 A. Vilà, M. González, and R. Mayol, Phys. Chem. Chem. Phys. 17, 32241 (2015).

257 A. Braun and M. Drabbels, J. Chem. Phys. 127, 114303 (2007).

258 A. Braun and M. Drabbels, J. Chem. Phys. 127, 114304 (2007).

259 A. Braun and M. Drabbels, J. Chem. Phys. 127, 114305 (2007).

260 A. Kautsch, M. Koch, and W. E. Ernst, Phys. Chem. Chem. Phys. 17, 12310 (2015).

261 A. Garcia, X. Buelna, E. Popov, and J. Eloranta, J. Chem. Phys. 145, 124504 (2016).

262 G. E. Busch and K. R. Wilson, J. Chem. Phys. 56, 3638 (1972).

263 F. Lackner, G. Krois, M. Theisen, M. Koch, and W. E. Ernst, Phys. Chem. Chem. Phys. 13, 18781 (2011).

264 J. Reho, C. Callegari, J. Higgins, W. E. Ernst, K. K. Lehmann, and G. Scoles, Faraday Discuss. 108, $161(1997)$.

265 G. Auböck, J. Nagl, C. Callegari, and W. E. Ernst, Phys. Rev. Lett. 101, 035301 (2008).

266 M. Leino, A. Viel, and R. E. Zillich, J. Chem. Phys. 129, 184308 (2008).

267 J. von Vangerow, O. John, F. Stienkemeier, and M. Mudrich, J. Chem. Phys. 143, 034302 (2015).

268 F. Coppens, N. Halberstadt, A. Leal, M. Mudrich, M. Pi, F. Stienkemeier, J. von Vangerow, and M. Barranco 2017 unpublished results.

269 M. Barranco, F. Coppens, N. Halberstadt, A. Leal, M. Mudrich, M. Pi, F. Stienkemeier, and J. von Vangerow 2017 contribution to the Quantum Fluid Cluster Workshop, Obergürgl, Austria, June 6-9.

270 C. Giese, T. Mullins, B. Grüner, M. Weidemüller, F. Stienkemeier, and M. Mudrich, J. Chem. Phys. 137, 244307 (2012).

271 M. Leino, A. Viel, and R. E. Zillich, J. Chem. Phys. 134, 024316 (2011).

272 F. Ancilotto, M. Barranco, E. S. Hernández, A. Hernando, and M. Pi, Phys. Rev. B 79, 104514 (2009). 
A. Scheidemann, J. P. Toennies, and J. A. Northby, Phys. Rev. Lett. 64, 1899 (1990).

274 J. Harms, J. P. Toennies, and F. Dalfovo, Phys. Rev. B 58, 3341 (1998).

275 J. Harms, J. P. Toennies, M. Barranco, and M. Pi, Phys. Rev. B 63, 184513 (2001).

276 D. Eichenauer, A. Scheidemann, and J. P. Toennies, Z. Phys. D 8, 295 (1988).

277 E. Krotscheck and R. E. Zillich, Eur. Phys. J. D 43, 113 (2007).

278 E. Krotscheck and R. E. Zillich, Phys. Rev. B 77, 094507 (2008).

279 A. Leal, D. Mateo, A. Hernando, M. Pi, and M. Barranco, Phys. Chem. Chem. Phys. 16, 23206 (2014).

280 A. Vilà, M. González, and R. Mayol, Phys. Chem. Chem. Phys. 18, 2006 (2016).

281 F. Coppens, A. Leal, M. Barranco, N. Halberstadt, and M. Pi, J. Low Temp. Phys. 187, 439 (2017).

282 L. F. Gomez, E. Loginov, R. Sliter, and A. F. Vilesov, J. Chem. Phys. 135, 154201 (2011).

283 R. M. P. Tanyag, C. F. Jones, C. Bernando, S. M. O. O'Connell, D. Verma, and A. F. Vilesov, in Cold Chemistry: Molecular Scattering and Reactivity Near Absolute Zero, edited by A. Osterwalder and O. Dulieu (Royal Society of Chemistry, Cambridge, UK, 2017), Chap. 8, (to be published).

284 E. Cheng, M. W. Cole, W. F. Saam, and J. Treiner, Phys. Rev. Lett. 67, 1007 (1991).

285 P.Taborek and J. E. Rutledge, Phys. Rev. Lett. 68, 2184 (1992).

286 F. Ancilotto, M. Barranco, E. S. Hernández, and M. Pi, J. Low Temp. Phys. 157, 174 (2009).

287 E. S. Hernández, F. Ancilotto, M. Barranco, R. Mayol, and M. Pi, Phys. Rev. B 73, 245406 (2006).

288 M. P. de Lara-Castells, N. F. Aguirre, H. Stoll, A. O. Mitrushchenkov, D. Mateo, and M. Pi, J. Chem. Phys. 142, 131101 (2015).

289 E. Loginov, L. F. Gomez, and A. F. Vilesov, J. Phys. Chem. A 115, 7199 (2011).

290 G. E. Johnson, Q. Hu, and J. Laskin, Annu. Rev. Anal. Chem. 4, 83 (2011).

291 P. Thaler, A. Volk, F. Lackner, J. Steurer, D. Knez, W. Grogger, F. Hofer, and W. E. Ernst, Phys. Rev. B 90, 155442 (2014).

292 N. F. Aguirre, D. Mateo, A. O. Mitrushchenkov, M. Pi, and M. P. de Lara-Castells, J. Chem. Phys. 136, 124703 (2012).

293 M. P. de Lara-Castells, H. Stoll, B. Civalleri, M. Causà, E. Voloshina, A. O. Mitrushchenkov, and M. Pi, J. Chem. Phys. 141, 151102 (2014). 
294 A. Volk, P. Thaler, M. Koch, E. Fisslthaler, W. Grogger, and W. E. Ernst, J. Chem. Phys. 138, $214312(2013)$. 